\title{
MEASURE AND AREA
}

\section{HERBERT FEDERER}

1. Introduction. For a long time the efforts of many mathematicians have been directed toward the creation of a complete theory which extends the quantitative concepts of Euclidean geometry to general subsets of Euclidean $n$-space, $E_{n}$, and to mappings into $E_{n}$. The first and fundamental steps in the modern development of this subject were taken by Lebesgue and Carathéodory with the invention of the general theory of measure. Subsequently the main interest has shifted naturally to the specific geometric problems which arise when the new concepts are applied in the extension of classical analytic formalisms and in the structural study of sets and mappings.

Measures are functions defined on classes of point sets; areas are functions defined on classes of mappings.

The first four sections of this paper are mainly expository. They contain the definitions of certain measures of geometric interest; a discussion of the rectifiability, tangent planes, densities and projections of point sets; and a description of a very general form of the Gauss-Green Theorem which illustrates the scope of the theory and its applicability to classical problems. Pivotal in this whole structure is the relationship between the purely metric notion of Hausdorff measure and the group-theoretic concepts of integral geometry.

The remaining four sections of this paper serve to establish some new results concerning the area of mappings. The basic issue motivating these investigations is the validity of the principle that every area defined by any reasonable method is naturally representable as an integral of a multiplicity function with respect to a measure. This raises a difficult problem because Lebesgue, in introducing his particular area which has been used very successfully by many workers in the field, was guided entirely by the analytic requirement of semi-continuity; his definition appears to have no immediate connection with the geometric measure theory which was created later by Carathéodory. This difficulty has now been overcome for the case of prime classical interest: mappings of a two-cell into three-space.

An address delivered before the New York meeting of the Society on April 27, 1951 by invitation of the Committee to Select Hour Speakers for Eastern Sectional Meetings; received by the editors November 13, 1951 and, with additions, January 21, 1952. This memoir, much longer than the usual invited address, is published in the Bulletin by special arrangement with the Council and the Board of Trustees. 
The principal new results are contained in the theorems $8.17,8.15$, and 6.9.

2. Definitions of some $k$-dimensional measures over $n$-space. Suppose $k \leqq n$ are positive integers, $\mathcal{L}_{k}$ is the $k$-dimensional Lebesgue measure over $E_{k}$, and

$$
\alpha(k)=\mathcal{L}_{k}\left(E_{k} \cap\{x|| x \mid<1\}\right)
$$

is the volume of the $k$-dimensional unit sphere.

Let $A \subset E_{n}$. With each positive number $r$ we associate the infimum of all numbers of the form

$$
\sum_{i=1}^{\infty} 2^{-k} \alpha(k)\left(\text { diameter } B_{i}\right)^{k}
$$

where

$$
\begin{aligned}
& A \subset \bigcup_{i=1}^{\infty} B_{i}, \\
& \text { diameter } B_{i}<r \quad \text { for } i=1,2,3, \cdots .
\end{aligned}
$$

As $r$ decreases toward zero, this infimum never decreases; it approaches the limit

$$
\mathfrak{H C}_{n}^{k}(A)
$$

the $k$-dimensional Hausdorff measure of the subset $A$ of $E_{n}$.

If $k=n$, then $\mathcal{H}_{n}^{k}=\mathcal{L}_{n}$ and the above infimum is actually independent of $r$. Carathéodory was the first to recognize the necessity of the double limiting process in case $k<n$. By attaching to each set $B_{i}$ a constant times the $k$ th power of its diameter, Hausdorff modified the earlier definition of Carathéodory, who had required $B_{i}$ to be convex and had associated with it the supremum of the $\mathcal{L}_{k}$ measures of its orthogonal projections into $E_{k}$. The measures of Carathéodory and Hausdorff are not identical, but their ratio is bounded [MEF 1]. This implies that the structure theorems for sets of finite Hausdorff measure, described in the following section, apply equally to sets of finite Carathéodory measure. However Hausdorff measure appears to be usually easier to work with and it has the advantage, exploited in $\$ 6$, that its definition remains meaningful for arbitrary metric spaces.

The simplest orthogonal projection of $E_{n}$ onto $E_{k}$ is the function $p_{\%}^{k}$ on $E_{n}$ such that

$$
p_{n}^{k}(x)=\left(x_{1}, \cdots, x_{k}\right) \in E_{k} \quad \text { for } \quad x=\left(x_{1}, \cdots, x_{n}\right) \in E_{n}
$$


Every orthogonal projection of $E_{n}$ onto $E_{k}$ is a superposition of the form

$$
p_{n}^{k} \circ R,
$$

where $R$ is an element of the group $G_{n}$ of orthogonal transformations of $E_{n}$.

For each function $f$, each set $X$, and each point $y$, let

$$
N(f, X, y)
$$

be the number (possibly $\infty$ ) of elements of the set

$$
X \cap\{x \mid f(x)=y\} .
$$

Now let $A$ be an analytic subset of $E_{n}$. For each $R \in G_{n}$ the integral

$$
\int_{E_{k}} N\left(A, p_{n}^{k} \circ R, y\right) d \mathcal{L}_{k} y
$$

indicates the size of the corresponding orthogonal projection of $A$, counting each point in $E_{k}$ with its multiplicity of projection. Using the Haar measure $\phi_{n}$ over $G_{n}$ for which $\phi_{n}\left(G_{n}\right)=1$ and the constant

$$
\beta(n, k)=\frac{\alpha(k) \cdot \alpha(n-k)}{\alpha(n) \cdot\left(\begin{array}{l}
n \\
k
\end{array}\right)},
$$

we define the integralgeometric Favard measure

$$
\mathcal{F}_{n}^{k}(A)=\beta(n, k)^{-1} \cdot \int_{G_{n}} \int_{E k} N\left(p_{n}^{k} \circ R, A, y\right) d \mathcal{L}_{k} y d \phi_{n} R
$$

as a kind of average projection size of $A$. In his note in the Comptes Rendus Favard attributes the idea for this definition-more precisely, a trivially equivalent definition - to a suggestion by Lebesgue. After that Favard never did anything to exploit this concept, whose fundamental importance will become clear in the next section.

The assumption that $A$ be an analytic set was made in order to assure the measurability of the integrand in the definition of $\mathcal{F}_{n}^{k}(A)$. The measure may be extended to arbitrary subsets $B$ of $E_{n}$ in such a way that $\mathcal{F}_{n}^{k}(B)$ is the infimum of $\mathcal{F}_{n}^{k}(A)$ for all analytic sets $A$ containing $B$.

The functions $\mathcal{F}_{n}^{k}$ and $\mathcal{F}_{n}^{k}$ are (Carathéodory outer) measures over $E_{n}$. Every closed subset of $E_{n}$ is both $\mathcal{F}_{n}^{k}$ and $\mathcal{F}_{n}^{k}$ measurable (in the sense of Carathéodory). Every subset of $E_{n}$ is contained in a $G_{\delta}$ set of equal $\mathfrak{K C}_{n}^{k}$ measure and also in an analytic set of equal $\mathfrak{F}_{n}^{k}$ measure. 
Every $\mathfrak{X C}_{n}^{k}$ measurable subset of finite $\mathfrak{J C}_{n}^{k}$ measure contains an $F_{\sigma}$ set of equal $\mathfrak{C C}_{n}^{k}$ measure. Every analytic set of finite $\mathcal{F}_{n}^{k}$ measure contains an $F_{\sigma}$ set of equal $\mathcal{F}_{n}^{k}$ measure.

If a function $g$ on $E_{n}$ to $\{t \mid-\infty \leqq t \leqq \infty\}$ is analytically measurable, which means that the $g$ counterimage of every open subset of the extended real number system is an analytic subset of $E_{n}$, then

$$
\int_{E_{n}} g(x) d \mathcal{F}_{n}^{k} x=\beta(n, k)^{-1} \int_{G_{n}} \int_{E_{k}} \sum_{\left(p_{n}^{k} \circ R\right)(x)=y} g(x) d \mathcal{L}_{k} y d \phi_{n} R .
$$

In view of the obvious additivity and convergence closure properties of the set of all functions $g$ for which this formula holds it is sufficient to observe that the formula is trivially true in the special case in which $g$ is the characteristic function of an analytic subset of $E_{n}$.

3. The structure of sets whose Hausdorff measure is finite. A function $f$ on $E_{k}$ to $E_{n}$ is said to be Lipschitzian if and only if there is a number $M$ such that

$$
\left|f(x)-f\left(x^{\prime}\right)\right| \leqq M \cdot\left|x-x^{\prime}\right| \quad \text { for } x \in E_{k} \text { and } x^{\prime} \in E_{k} .
$$

According to Rademacher, such a function $f$ has at $\mathcal{L}_{k}$ almost every point $x$ of $E_{k}$ a total (Fréchet) differential which is represented by a matrix of $k$ columns and $n$ rows of partial derivatives; the square root of the sum of the squares of the determinants of the $k$-rowed minors of this matrix is the Jacobian $J f(x)$. The connection between these analytic concepts and the measure theory of the preceding section is established by the formula

$$
\begin{aligned}
\int_{X} J f(x) d \mathcal{L}_{k} x & =\int_{E_{n}} N(f, X, y) d \mathcal{C}_{n}^{k} y \\
& =\int_{E_{n}} N(f, X, y) d \mathcal{F}_{n}^{k} y,
\end{aligned}
$$

which is valid for each Lipschitzian function on $E_{k}$ to $E_{n}$ and each $\mathcal{L}_{k}$ measurable set $X$ (see $[\mathrm{F} 1,3]$ ). The two integrals on the right are equal to the countable sums

$$
\sum_{i=1}^{\infty} i \cdot \mathfrak{F}_{n}^{k}(\{y \mid N(f, X, y)=i\})+\infty \cdot \mathfrak{F}_{n}^{k}(\{y \mid N(f, X, y)=\infty\})
$$

and

$$
\sum_{i=1}^{\infty} i \cdot \mathcal{F}_{n}^{k}(\{y \mid N(f, X, y)=i\})+\infty \cdot \mathcal{F}_{n}^{k}(\{y \mid N(f, X, y)=\infty\}) .
$$


We follow the usual convention of measure theory that $\infty \cdot 0=0$. In particular, if $f$ maps $X$ univalently onto $Y$, then

$$
\int_{X} J f(x) d \mathcal{L}_{k} x=\mathcal{F C}_{n}^{k}(Y)=\mathcal{F}_{n}^{k}(Y) .
$$

In this connection it is useful to know that every $\mathcal{L}_{k}$ measurable set $X$ contains an $\mathcal{L}_{k}$ measurable set $X^{\prime}$ such that $f$ is univalent on $X^{\prime}$ and such that the sets $X$ and $X^{\prime}$ have the same $f$ image ([MF]).

This leads us to consider those sets which are almost representable as Lipschitzian images of subsets of $E_{k}$. More precisely we say that a subset $A$ of $E_{n}$ is Hausdorff $k$ rectifiable if and only if there is a Lipschitzian function $f$ on $E_{k}$ to $E_{n}$ such that

$$
\mathcal{F C}_{n}^{k}(A-\text { range } f)=0 .
$$

Every subset of a Hausdorff $k$ rectifiable set is Hausdorff $k$ rectifiable. A countable union of Hausdorff $k$ rectifiable sets is Hausdorff $k$ rectifiable.

Before entering into a deeper discussion of Hausdorff $k$ rectifiable sets we must introduce some of the concepts which help to describe the local behavior of sets of finite Hausdorff measure. For $a \in E_{n}$ and $r>0$ we let

$$
K(a, r)=\{x|| x-a \mid<r\}
$$

be the open sphere with center $a$ and radius $r$. If $A$ is a regularly embedded $k$-dimensional manifold through $a$, then $\mathfrak{F C}_{n}^{k}[A \cap K(a, r)]$ is asymptotically equal to $\alpha(k) \cdot r^{k}$ as $r$ approaches zero. This suggests the definition, for arbitrary $A \subset E_{n}$ and $a \in E_{n}$, of the upper and lower Hausdorff $k$ densities

$$
\begin{aligned}
\odot_{n}^{k}(A, a) & =\limsup _{r \rightarrow 0+} \frac{\mathcal{F C}_{n}^{k}[A \cap K(a, r)]}{\alpha(k) r^{b}}, \\
\Theta_{n}^{k}(A, a) & =\liminf _{r \rightarrow 0+} \frac{\mathcal{F C}_{n}^{k}[A \cap K(a, r)]}{\alpha(k) r^{b}} .
\end{aligned}
$$

If $A$ is any set for which $\operatorname{SC}_{n}^{k}(A)<\infty$, then

$$
2^{-k} \leqq \odot_{n}^{k}(A, a) \leqq 1 \text { for } \mathcal{F}_{n}^{k} \text { almost all } a \text { in } A .
$$

If, in addition, the set $A$ is $\mathcal{F}_{n}^{k}$ measurable, then

$$
\bar{\odot}_{n}^{k}(A, a)=0 \text { for } \mathcal{B C}_{n}^{k} \text { almost all } a \text { in } E_{n}-A .
$$


There exist $\mathcal{H C}_{n}^{k}$ measurable sets $A$ for which $0<\mathcal{F C}_{n}^{k}(A)<\infty$ and $\mathcal{\odot}_{n}^{k}(A, a)=0$ for all $a \in E_{n}$.

Turning now to the subject of approximate tangent planes, we first observe that for each $R \in G_{n}$ the orthogonal projection $\left(p_{n}^{k} \circ R\right)$ maps the $k$-plane

$$
\left\{x|| x|=|\left(p_{n}^{k} \circ R\right)(x) \mid\right\}
$$

isometrically onto $E_{k}$. The $k$-plane parallel to the above and through the point $a \in E_{n}$ is

$$
\square_{n}^{k}(R, a)=\left\{x|| x-a|=|\left(p_{n}^{k} \circ R\right)(x-a) \mid\right\} .
$$

By saying that the $k$-plane $\square_{n}^{k}(R, a)$ is an approximate tangent plane of the set $A \subset E_{n}$ at the point $a$ we mean roughly that most of those points of $A$ which are close to $a$-say in $K(a, r)$-lie in a suitably preassigned neighborhood of $\square_{n}^{k}(R, a)-\{a\}$. For the latter we choose the sets

$$
\diamond_{n}^{k}(R, \epsilon, a)=\left\{x|| x-a\left|<\left(1+\epsilon^{2}\right)^{1 / 2}\right|\left(p_{n}^{k} \circ R\right)(x-a) \mid\right\}
$$

corresponding to $\epsilon>0$. A point $x$ of $E_{n}$ is in $\diamond_{n}^{k}(R, \epsilon, a)$ if and only if the distance from $x$ to the $(n-k)$-plane through $a$ and perpendicular to $\square_{n}^{k}(R, a)$ is less than $\epsilon$ times the distance from $x$ to $\square_{n}^{k}(R, a)$. Requiring of course that $A$ should appreciably enter into arbitrarily small neighborhoods of $a$, we phrase the precise definition as follows: The set $A \subset E_{n}$ has at the point $a \in E_{n}$ the Hausdorff $k$ (approximate) tangent plane $\square_{n}^{k}(R, a)$ if and only if

and

$$
\odot_{n}^{k}(A, a)>0
$$

$$
\odot_{n}^{k}\left[A-\diamond_{n}^{k}(R, \epsilon, a), a\right]=0 \text { for every } \epsilon>0 .
$$

Under these circumstances we shall refer to $\left(p_{n}^{k} \circ R\right)$ as a tangential projection of $A$ at $a$. It is clear that $R$ is not uniquely determined by $\square_{n}^{k}(R, a)$ and serves only as a convenient parameter, nor is the tangential projection uniquely determined by $\square_{n}^{k}(R, a)$. However the $k$-plane $\square_{n}^{k}(R, a)$ is usually unique in cases of interest to us, for if $\mathfrak{F C}_{n}^{k}(A)<\infty$, then $A$ has at $\mathfrak{H}_{n}^{k}$ almost all points of $E_{n}$ at most one Hausdorff $k$ tangent plane, though it need not have any.

Fixing $\epsilon>0$, we observe that

$$
\bar{\odot}_{n}^{k}\left[A-\diamond_{n}^{k}(R, \epsilon, a), a\right\rfloor=\limsup _{r \rightarrow 0+} \frac{\mathcal{H}_{n}^{k}\left[A \cap K(a, r)-\diamond_{n}^{k}(R, \epsilon, a)\right]}{\alpha(k) r^{k}} .
$$


If $A$ is a regularly embedded $k$-dimensional manifold through $a$ whose tangent plane at $a$ in the classical sense is $\square_{n}^{\mathfrak{k}}(R, a)$, then the set whose Hausdorff measure occurs in the numerator is vacuous whenever $r$ is sufficiently small. The notion of an approximate tangent plane is therefore an asymptotic generalization of the corresponding classical concept.

We have now filled in the background necessary for the understanding of the following basic theorem describing the structure of sets whose Hausdorff measure is finite.

Suppose $A$ is an $\mathfrak{F C}_{n}^{k}$ measurable set for which

$$
\mathfrak{H C}_{n}^{k}(A)<\infty,
$$

$B$ is the set of all those points of $A$ at which $A$ has a Hausdorff $k$ tangent plane, and

$$
C=A-B
$$

Then:

(1) $B$ and $C$ are $\Im_{n}^{k}$ measurable sets.

(2) $B$ is Hausdorff $k$ rectifiable.

(3) C contains no Hausdorff $k$ rectifiable set of positive $\mathrm{FC}_{n}^{k}$ measure.

(4) $\mathcal{F}_{n}^{k}(B)=\mathfrak{F}_{n}^{k}(B)$.

(5) $\mathcal{F}_{n}^{\mathfrak{k}}(C)=0$; for $\phi_{n}$ almost all $R$ in $G_{n}$ the $\left(p_{n}^{k} \circ R\right)$ image of $C$ has $\mathcal{L}_{k}$ measure zero.

(6) $\mathfrak{F C}_{n}^{k}(A)=\mathfrak{H C}_{n}^{k}(B)+\mathfrak{H C}_{n}^{k}(C)=\mathcal{F}_{n}^{k}(B)+\mathcal{F C}_{n}^{k}(C)=\mathcal{F}_{n}^{k}(A)+\mathcal{K}_{n}^{k}(C)$.

(7) $\mathcal{F}_{n}^{k}(A) \leqq \mathfrak{K C}_{n}^{k}(A)$.

(8) The following three conditions are equivalent:

(i) $\mathcal{F}_{n}^{\mathfrak{k}}(A)=\mathfrak{H C}_{n}^{\mathfrak{k}}(A)$.

(ii) $A$ is Hausdorff $k$ rectifiable.

(iii) $A$ has Hausdorff $k$ tangent planes at $\mathcal{F}_{n}^{k}$ almost all of its points.

(9) The following three conditions are equivalent:

(i) $\mathcal{F}_{n}^{k}(A)=0$.

(ii) A contains no Hausdorff $k$ rectifiable set of positive $\mathfrak{H C}_{n}^{\mathrm{k}}$ measure.

(iii) A has Hausdorff $k$ tangent planes at $\mathfrak{F C}_{n}^{k}$ almost none of its points.

For the special case in which $k=1$ and $n=2$ these results are substantially due to A. S. Besicovitch, A. P. Morse, and J. F. Randolph ([B 1$]$, [MR]). The extension to general dimensions was made by the writer ([F 4]).

Intimately related to this structure theory, there has been a great deal of research into the subject of Hausdorff densities. It is easy to prove that

$$
\bar{\odot}_{n}^{k}(A, a)=\varrho_{n}^{k}(A, a)=1 \text { for } \mathcal{C}_{n}^{k} \text { almost all } a \text { in } B,
$$


but the behavior of the densities on $C$ presents a difficult problem. For the special case in which $k=1$ it is known that

$$
\bar{\odot}_{n}^{1}(A, a) \geqq 1.01 \odot_{n}^{1}(A, a) \text { for } \mathcal{F C}_{n}^{1} \text { almost all } a \text { in } C .
$$

This intriguing estimate was obtained by A. P. Morse and J. F. Randolph for $n=2$ and extended by E. F. Moore to all positive integers $n$ (See [MR], [MEF 2]).

4. The Gauss-Green Theorem. The classical formula bearing this name asserts that the integral of a partial derivative of a function over an open subset of $E_{n}$ equals the integral of the function itself, multiplied by a component of the exterior normal, over the boundary of the open set. Giving precise measure-theoretic interpretations to the intuitive concepts which enter into this statement, we are able to give it a completely natural and general form.

Suppose $U$ is a bounded open subset of $E_{n}, A$ is the boundary of $U$, and $\mathfrak{H C}_{n}^{n-1}(A)<\infty$.

We shall integrate with respect to $\mathcal{L}_{n}$ over $U$ and with respect to $\mathcal{H}_{n}^{n-1}$ over $A$. Our definition of the term "exterior normal" is as follows: Suppose $a \in A$ and $w$ is a unit vector, that is, $w \in E_{n}$ and $|w|$ $=1$. For each $r>0$ we consider the solid hemispheres

$$
\begin{aligned}
& s(r)=K(a, r) \cap\{x \mid(x-a) \bullet w \geqq 0\}, \\
& t(r)=K(a, r) \cap\{x \mid(x-a) \bullet w \leqq 0\}
\end{aligned}
$$

into which the sphere $K(a, r)$ is divided by the $(n-1)$-plane through $a$ and perpendicular to $w$. The vector $w$ points from $a$ into $s(r)$, and $-w$ points into $t(r)$. By saying that $w$ is an exterior normal of $U$ at $a$ we mean roughly that for small $r$ the hemisphere $t(r)$ is filled mostly with points of $U$ while $s(r)$ contains only few points of $U$; precisely we mean that

$$
\lim _{r \rightarrow 0+} \frac{\mathcal{L}_{n}[t(r) \cap U]}{\mathcal{L}_{n}[t(r)]}=1
$$

and

$$
\lim _{r \rightarrow 0+} \frac{\mathcal{L}_{n}[s(r) \cap U]}{\mathcal{L}_{n}[s(r)]}=0 .
$$

The set $U$ has at each point $a$ at most one exterior normal, but it may have none. We define

$$
\nu(U, a)
$$


as the exterior normal of $U$ at $a$ whenever that exists, and as the zero vector otherwise. We also let $\nu_{j}(U, a)$ be the $j$ th component of the vector $\nu(U, a)$ for $j=1,2, \cdots, n$.

From our discussion of the geometric properties of the domains of integration we now turn to the assumptions about the function which is to occur in our formula.

Suppose $f$ is a numerically-valued function, continuous on $U \cup A$, and absolutely continuous in the sense of Tonelli on $U$.

The last part of this hypothesis means that for $j=1,2, \cdots, n$ the partial derivative

$$
D_{i} f(x)
$$

of $f$ in the direction of the $j$ th base vector exists for $\mathcal{L}_{n}$ almost all $x$; that

$$
\int_{U}\left|D_{i} f(x)\right| d \mathcal{L}_{n} x<\infty ;
$$

and that for $\mathcal{L}_{n-1}$ almost all $y$ in $E_{n-1}$ the function

$$
f\left(y_{1}, \cdots, y_{i-1}, z, y_{j+1}, \cdots, y_{n-1}\right)
$$

of one variable $z$ is absolutely continuous in the classical sense on every interval $I$ for which

$$
\left\{\left(y_{1}, \cdots, y_{j-1}, z, y_{j+1}, \cdots, y_{n-1}\right) \mid z \in I\right\} \subset U .
$$

We observe that for all this to hold it would be sufficient, but not necessary, to assume that the first partial derivatives of $f$ are continuous and bounded on $U$. Under the foregoing assumptions the writer has proved (in [F 2, 3]) the Gauss-Green formula:

$$
\int_{U} D_{j} f(x) d \mathcal{L}_{n} x=\int_{A} f(a) \nu_{j}(U, a) d \mathfrak{F C}_{n}^{n-1} a \quad \text { for } j=1,2, \cdots, n .
$$

Inasmuch as all earlier definitions of exterior normals utilized tangent planes of the boundary set, it is interesting to note that at $\mathfrak{H C}_{n}^{n-1}$ almost all of those points $a$ of $A$ at which $U$ has an exterior normal the $(n-1)$-plane

$$
\{x \mid(x-a) \bullet \nu(A, a)=0\}
$$

is a Hausdorff $n-1$ tangent plane of $A$ at $a$. The converse is false, as may be seen from the simple example in which $n=2$ and $U$ is an open disc minus a radius; at each point of this radius the set $A$ has a Hausdorff 1 tangent plane, but $U$ has no exterior normal. 
The set of those points of $A$ at which $U$ has no exterior normal contributes nothing to the integral on the right, because $\nu_{j}(U, a)=0$ for all such points $a$. However this point set and, worse than that, the set of those points of $A$ at which $A$ has no Hausdorff $n-1$ tangent plane may very well have positive $\mathfrak{H C}_{n}^{n-1}$ measure. This situation may occur even in the relatively simple case in which $n=3$ and $A$ is homeomorphic with a two sphere. Significant in this connection is the fact that, while every connected set of finite ${ }^{2} \mathrm{C}_{n}^{1}$ measure is Hausdorff 1 rectifiable, the intrinsic topological properties of a set of finite $\mathcal{F C}_{n}^{k}$ measure have no bearing whatever on its rectifiability in case $k>1$.

To our version of the Gauss-Green formula corresponds the following form of the Cauchy Theorem:

If $U$ is a bounded set of complex numbers, $A$ is the boundary of $U$, ${ }^{\mathrm{C}_{2}^{1}}(A)<\infty$, and if the complex-valued function $f$ is continuous on $U \cup A$ and analytic on $U$, then

$$
\int_{A} f(a) \nu(U, a) d \mathcal{F}_{2}^{1} a=0 .
$$

This reduces to the classical formula in case $A$ is a simple closed rectifiable curve in the plane.

5. Multiplicity functions and areas. Assuming that $X$ is a locally compact, locally connected, separable subset of a triangulable $k$-dimensional manifold we let

$$
C_{n}(X)
$$

be the set of all continuous functions on $X$ to $E_{n}$. We wish to associate with each mapping $f \in C_{n}(X)$ a non-negative number (possibly $\infty$ ) indicating the $k$-dimensional extent of the point set occupied by the values of $f$ as well as the multiply overlapping manner in which the various parts of this point set may be covered by $f$. If such an association has invariance properties consistent with the Euclidean geometry of $E_{n}$, and if it relates to relatively simple (differentiable) mappings the same numbers as do the classical integral formulae, then we shall refer to it as a $k$-dimensional area on $C_{n}(X)$. No precise definition of this general concept of area, which would complement Carathéodory's axiomatization of the notion of measure, has yet been devised. Up to now all the work in this field has been directed toward the study and comparison of certain particular functions which belong to the still vague category of all areas. In the remainder of the present paper we shall continue these special investigations, 
but we shall point out some desirable general properties of areas as they occur in our work.

Most areas are defined as integrals of multiplicity functions by the following scheme: Suppose $\mu$ is such a function that, for $f \in C_{n}(X)$ and $y \in E_{n}$,

$$
\mu(f, X, y)
$$

is either a non-negative integer or $\infty$ and gives a sensible appraisal of the multiplicity with which the mapping $f$ assumes the value $y$. Suppose further that $\psi$ is a measure over $E_{n}$ and is reasonably indicative of $k$-dimensional Euclidean extent. Then the function which associates

$$
\int_{E_{n}} \mu(f, X, y) d \psi y
$$

with $f \in C_{n}(X)$ is the area on $C_{n}(X)$ corresponding to the multiplicity function $\mu$ and the measure $\psi$.

In $\$ 2$ we have already described the elementary multiplicity function $N$ which simply counts the points $x$ in $X$ for which $f(x)=y$. Used in conjunction with the measure $\mathcal{F C}_{n}^{k}$ it yields the k-dimensional Hausdorff $N$ area

$$
\int_{E_{n}} N(f, X, y) d \mathcal{K}_{n}^{k} y,
$$

and with $\mathcal{F}_{n}^{k}$ the $k$-dimensional integralgeometric $N$ area

$$
\int_{E_{n}} N(f, X, y) d \mathcal{F}_{n}^{k} y,
$$

of the mapping $f \in C_{n}(X)$. According to $\S 3$ these areas agree with the classical integral formula in case $X$ is an $\mathcal{L}_{k}$ measurable subset of $E_{k}$ and $f$ is a Lipschitzian mapping. For any $X$ and any $f \in C_{n}(X)$ our structure theorem concerning sets of finite Hausdorff measure implies that

$$
\int_{E_{n}} N(f, X, y) d \mathcal{C}_{n}^{k} y \geqq \int_{E_{n}} N(f, X, y) d \mathcal{F}_{n}^{k} y
$$

and that, in case the $k$-dimensional Hausdorff area of $f$ is finite, equality holds if and only if the range of $f$ is a Hausdorff $k$ rectifiable set. A comprehensive list of such inequalities, containing all known results of this type except for those derived in the following sections of this paper, may be found in the writer's recent note [ $\mathrm{F}$ 6]. 
Assuming that $\psi$ is such a measure over $E_{n}$ that all closed subsets of $E_{n}$ are $\psi$ measurable, the $\psi N$ area of any $f \in C_{n}(X)$ may (according to [F 1]) be computed as follows: Let a partition $P$ of $X$ be such a countable disjointed family of analytic subsets of $X$ that

$$
\underset{W \in P}{\bigcup} W=X
$$

and let

$$
\sigma(\psi, f, P)=\sum_{W \in P} \psi[f(W) .]
$$

Then the $\psi N$ area

$$
\int_{E_{n}} N(f, X, y) d \psi y
$$

of $f$ equals the supremum of $\sigma(\psi, f, P)$ for all partitions $P$ of $X$; it also equals

$$
\lim _{i \rightarrow \infty} \sigma\left(\psi, f, P_{i}\right)
$$

whenever $P_{1}, P_{2}, P_{3}, \cdots$ are such partitions of $X$ that

$$
\lim _{i \rightarrow \infty} \sup _{W \in_{P_{i}}} \operatorname{diameter} W=0 .
$$

These alternate methods for the computation of the $N$ area embody the general measure-theoretic principle underlying the classical theorem which states that the length of a continuous curve, defined as the supremum of the lengths of all inscribed polygons, equals the limit of the lengths of the terms of any sequence of inscribed polygons with vertices corresponding to finer and finer subdivisions of the interval on which the curve is parametrically represented. In this case $\psi$ is $\mathcal{F}_{n}^{1}$ or $\mathcal{F}_{n}^{1}, X$ is a closed interval, and only finite partitions of $X$ into subintervals need be considered. The classical length of any curve $f \in C_{n}(X)$ is found to be equal to the one-dimensional Hausdorff $N$ area of $f$ and also to the one-dimensional integralgeometric $N$ area of $f$.

Among the elementary properties of the multiplicity function $N$ the following three appear to have some general significance:

(i) Localizability of $N$. If $X^{\prime} \subset X$ and

$$
X \cap\{x \mid f(x)=y\} \subset X^{\prime},
$$




$$
N(f, X, y)=N\left(f, X^{\prime}, y\right) .
$$

(ii) Additivity of N. If $P$ is such a disjointed family of subsets of $X$ that

$$
\bigcup_{W \in P} W=X
$$

then

$$
N(f, X, y)=\sum_{W \in P} N(f, W, y) .
$$

(iii) Superposition formula for $N$. If

$$
Y_{i}=\{y \mid N(f, X, y) \geqq i\} \quad \text { for } i=1,2,3, \cdots,
$$

then

$$
N(g \circ f, X, z)=\sum_{i=1}^{\infty} N\left(g, Y_{i}, z\right)
$$

Analogous, somewhat weaker properties are shared by all those functions to which we vaguely refer in this paper as "multiplicity functions," and will certainly play a role in any future precise definition of that term.

The superposition formula for $N$ may be used to show that the $k$-dimensional integralgeometric $N$ area of $f \in C_{n}(X)$ equals

$$
\beta(n, k)^{-1} \cdot \int_{G_{n}} \int_{E_{k}} N\left(p_{n}^{k} \circ R \circ f, X, z\right) d \mathcal{L}_{k} z d \phi_{n} R,
$$

a kind of average over $G_{n}$ of the areas of the orthogonal projections $p_{n}^{k} \circ R \circ f$ of $f$. In case $X$ is $k$-dimensional and $k<n$ these projections, which map $X$ into $E_{k}$, are much easier to study than the mapping $f$ of $X$ into $E_{n}$. In this respect the integralgeometric area is simpler than the Hausdorff area, which cannot be represented as an average of this type.

There are several natural multiplicity functions other than $N$. Among these the function $S$ is connected with the concept of stability. We recall that $y$ is said to be a stable value of $f$ if and only if $y$ is in the range of every mapping sufficiently close to $f$. The underlying topology on $C_{n}(X)$ is based on the neighborhoods

$$
C_{n}(X) \cap\{g|| g(x)-f(x) \mid<\epsilon \text { for } x \in X\}
$$

of $f$ corresponding to $\epsilon>0$. Convergence in this topology is uniform on $X$. Introducing (as in [R 3]) the stable multiplicity 


$$
S(f, X, y)=\liminf _{0 \rightarrow f} N(g, X, y),
$$

we note that $y$ is a stable value of $f$ if and only if $S(f, X, y)>0$. We also observe that

$$
\limsup _{o \rightarrow f} N(g, X, y)=\infty
$$

whenever $X$ is dense in itself and $y$ is in the closure of the range of $f$. Since $X$ is at most $k$-dimensional, $S(f, X, y)=0$ in case $k<n$. Therefore the function $S$ is directly useful only in case $k=n$, where it gives rise to the $\mathcal{L}_{k} S$ area on $C_{k}(X)$. However the alternate formula for the integralgeometric $N$ area, which was discussed in the preceding paragraph, suggests the indirect definition of the $k$-dimensional integralgeometric stable area on $C_{n}(X)$ associating

$$
\beta(n, k)^{-1} \int_{G_{n}} \int_{E_{k}} S\left(p_{n}^{k} \circ R \circ f, X, z\right) d \mathcal{L}_{k} z d \phi_{n} R
$$

with $f \in C_{n}(X)$ for $k \leqq n$. In case $X$ is a subset of $E_{k}$ whose boundary has $\mathcal{L}_{k}$ measure zero and $f$ is a Lipschitzian mapping this stable area of $f$ agrees (according to [F 3 ]) with the classical Jacobian integral formula, because each projection $p_{n}^{k} \circ R \circ f$ is a Lipschitzian mapping in $C_{k}(X)$ and because

$$
S(h, X, z)=N(h, X, z) \text { for } \mathcal{L}_{k} \text { almost all } z \text { in } E_{k}
$$

whenever $h$ is a Lipschitzian mapping in $C_{k}(X)$. Furthermore we have

$$
S(h, X, z) \leqq N(h, X, z) \text { for } h \in C_{k}(X) \text { and } z \in E_{k},
$$

whence we conclude that the $k$-dimensional integralgeometric stable area of every $f \in C_{n}(X)$ is less than or equal to the $k$-dimensional integralgeometric $N$ area of $f$.

From the point of view of approximation theory the function $N$, which accounts only for the relatively accidental behavior of an individual mapping, is less appropriate than the stable multiplicity function $S$. Indeed $S(f, X, y)$ is computable by the Cech cohomology theory which approximates $X$ and $f$ by complexes and simplicial mappings [F 7].

For $f \in C_{k}(X), y \in E_{k}$, and $r>0$ the components of the open set

$$
X \cap\{x|| f(x)-y \mid<r\}
$$

are connected open sets. We shall refer to them as canonical regions of $(f, X, y, r)$. Furthermore we shall call $F$ a canonical family of $(f, X, y, \epsilon)$ if and only if each element of $F$ is a canonical region of 
$(f, X, y, r)$ for some $r \leqq \epsilon, F$ is disjointed, $F$ covers the closed set

$$
X \cap\{x \mid f(x)=y\},
$$

and every point of $X$ has a neighborhood which meets only finitely many elements of $F$.

If $f \in C_{k}(X), y \in E_{k}$, and $\epsilon>0$, then there exists a canonical family $F$ of $(f, X, y, \epsilon)$.

To prove this we let

$$
\begin{aligned}
A & =\{x \mid f(x)=y\}, \\
G(r) & =\text { the set of all canonical regions of }(f, X, y, r)
\end{aligned}
$$

whenever $r>0$, choose compact subsets

$$
0=B_{0} \subset B_{1} \subset B_{2} \subset \cdots
$$

of $X$ for which

$$
X=\bigcup_{i=1}^{\infty} \text { Interior } B_{i},
$$

and inductively define the sequences

$$
r_{0}, r_{1}, r_{2}, \cdots ; H_{0}, H_{1}, H_{2}, \cdots ; U_{0}, U_{1}, U_{2}, \cdots
$$

in such a way that

$$
r_{0}=\epsilon, \quad H_{0}=0, \quad U_{0}=0
$$

and

$$
\begin{aligned}
r_{i} & =\inf \left(\left\{r_{i-1}\right\} \cup\left\{|f(x)-y| \mid x \in B_{i-1}-U_{i-1}\right\}\right), \\
H_{i} & =G\left(r_{i}\right) \cap\left\{V \mid V \cap A \cap B_{i} \nsubseteq U_{i-1}\right\}, \\
U_{i} & =U_{i-1} \cup \bigcup_{V \in H_{i}} V
\end{aligned}
$$

for $i=1,2,3, \ldots$. We shall prove that

$$
F=\bigcup_{i=1}^{\infty} H_{i}
$$

is a canonical family of $(f, X, y, \epsilon)$.

First we verify by induction that

$$
0<r_{i} \leqq \epsilon \text { and }\left(A \cap B_{i}\right) \subset U_{i}
$$

for every non-negative integer $i$. The statement holds if $i=0$. If it holds for $i=j-1$, then

$$
\left(A \cap B_{j-1}\right) \subset U_{j-1}, \quad\left(B_{i-1}-U_{j-1}\right) \cap A=0,
$$




$$
\begin{gathered}
|f(x)-y|>0 \text { for } x \in B_{j-1}-U_{j-1}, \\
B_{j-1}-U_{j-1} \text { is compact, } 0<r_{j} \leqq r_{j-1}, \\
A \subset\left\{x|| f(x)-y \mid<r_{j}\right\} \subset \underset{V \in U_{G\left(r_{j}\right)} V,}{\bigcup} V, \quad\left(A \cap B_{j}\right) \subset U_{j},
\end{gathered}
$$

whence the statement holds for $i=j$.

We infer that each element of $F$ is a canonical region of $\left(f, X, y, r_{i}\right)$ for some $r_{i} \leqq \epsilon$, and that $F$ covers $A$.

Since $G(r)$ is disjointed for $r>0, H_{i}$ is disjointed for $i=1,2,3, \cdots$. If $F$ were not disjointed, there would exist $i<j, V \in H_{i}, W \in H_{j}$ with $V \cap W \neq 0$; hence $r_{j} \leqq r_{i}, V \in G\left(r_{i}\right), W \in G\left(r_{j}\right), W \subset V \subset U_{i} \subset U_{j-1}$, $W \notin H_{j}$. It follows that $F$ is disjointed.

For $i=1,2,3, \cdots$ the set $H_{i}$ is a disjointed family of open sets each of which meets the compact set $B_{i}$; hence $H_{i}$ is finite. In view of this and of the fact that every point of $X$ is interior to some set $B_{j}$, we may complete the proof by checking that

$$
F \cap\left\{V \mid V \cap B_{i} \neq 0\right\} \subset \bigcup_{i=1}^{j} H_{i} \text { for } j=1,2,3, \cdots .
$$

To do this suppose $i<j, V \in H_{j}, x \in V \cap B_{i}$. Then

$$
\begin{gathered}
x \in V \in G\left(r_{j}\right), \quad|f(x)-y|<r_{j}, \\
V \cap U_{i}=0, \quad x \in B_{i}-U_{i}, \quad|f(x)-y| \geqq r_{i+1},
\end{gathered}
$$

whence $r_{i+1}<r_{j}$, contrary to the fact that $i+1 \leqq j$ and $r_{i+1} \geqq r_{j}$.

The proof is complete.

A canonical region $V$ of $(f, X, y, r)$ is said to be inessential if and only if there exists a mapping

$$
v \in C_{k}(\text { Closure } V)
$$

such that

$$
\begin{aligned}
v(x) & =f(x) \text { for } x \in \text { Boundary } V, \\
|v(x)-y| & =r \text { for } x \in V .
\end{aligned}
$$

Otherwise $V$ is essential; in this case there exists a mapping

$$
v \in C_{k}(\text { Closure } V)
$$

such that

$$
v(x)=f(x) \text { for } x \in \text { Boundary } V,
$$




$$
\begin{aligned}
|v(x)-y| \leqq r \text { for } x \in V, & \\
N(v, V, y) & =1 .
\end{aligned}
$$

Corresponding to each canonical family $F$ of $(f, X, y, \epsilon)$ we construct a function $g$ by choosing for each region $V \in F$ a mapping $v \in C_{k}$ (Closure $V$ ) as above, letting $g(x)=v(x)$ for $x \in V$, and letting

$$
g(x)=f(x) \text { for } x \in X-\bigcup_{V \in F} V .
$$

The locally finite character of $F$ assures us that $g \in C_{k}(X)$. Furthermore

$$
\begin{gathered}
|g(x)-f(x)| \leqq 2 \epsilon \text { for } x \in X \\
N(g, X, y)=\text { the number of essential regions in } F .
\end{gathered}
$$

With this machinery at hand we proceed to the proof of the following proposition:

If $f \in C_{k}(X), y \in E_{k}$, and

$$
s(r)=\text { the number of essential canonical regions of }(f, X, y, r)
$$

whenever $r>0$, then

$$
\lim _{r \rightarrow 0+} s(r)=S(f, X, y) .
$$

The above limit exists because $r>r^{\prime}>0$ implies that every essential canonical region of $(f, X, y, r)$ contains an essential canonical region of $\left(f, X, y, r^{\prime}\right)$, whence $s(r) \leqq s\left(r^{\prime}\right)$.

If $g \in C_{k}(X), r>0$, and

$$
|g(x)-f(x)|<r \text { for } x \in X,
$$

then $N(g, V, y) \geqq 1$ for every essential canonical region $V$ of $(f, X, y, r)$, because $f$ and $g$ define homotopic mappings of Boundary $V$ into $E_{k}-\{y\}$; it follows that $N(g, X, r) \geqq s(r)$. Consequently

$$
S(f, X, y) \geqq \lim _{r \rightarrow 0+} s(r) \text {. }
$$

In proving the opposite inequality we may assume that there exist a positive number $\rho$ and an integer $i$ such that

$$
s(r)=i \text { for } 0<r \leqq \rho .
$$

Suppose $\epsilon>0$ and let $F$ be a canonical family of $(f, X, y, \epsilon)$. If $V_{1}, V_{2}, \cdots, V_{j}$ are essential regions in $F$, let $r_{1}, r_{2}, \cdots, r_{j}$ and $r$ be such numbers that 


$$
0<r \leqq \rho, r \leqq r_{t},
$$

and

$V_{t}$ is a canonical region of $\left(f, X, y, r_{t}\right)$ for $t=1,2, \cdots, j$.

Then each $V_{t}$ contains an essential canonical region of $(f, X, y, r)$, whence $j \leqq i$. Constructing $g$ corresponding to $F$ as previously indicated, we find that $g \in C_{k}(X)$,

$$
\begin{aligned}
|g(x)-f(x)| & \leqq 2 \epsilon \text { for } x \in X, \\
N(g, X, y) & \leqq i .
\end{aligned}
$$

It follows that $S(f, X, y) \leqq i$.

The proposition whose proof we have just completed reduces the computation of the stable multiplicity to the counting of essential canonical regions. The Hopf extension theorem [D] of combinatorial topology provides an apt criterion to decide whether a canonical region $V$ of $(f, X, y, r)$ is essential. Since $f$ maps Closure $V$ into $E_{k}$ and Boundary $V$ into $E_{k} \cap\{z|| z-y \mid \geqq r\}, f$ induces a homomorphism

$$
f^{*}: H^{k}\left(E_{k}, E_{k} \cap\{z|| z-y \mid \geqq r\}\right) \rightarrow H^{k}(\text { Closure } V \text {, Boundary } V)
$$

of the $k$-dimensional Cech cohomology groups with integer coefficients and based on locally finite coverings. A necessary and sufficient condition for $V$ to be inessential is that $f^{*}$ be a trivial homomorphism (whose range has only one element).

The preceding condition is certainly satisfied whenever $H^{k}$ (Closure $V$, Boundary $V$ ) is a trivial group (with only one element). This is actually the case unless $V$ is a $k$-dimensional manifold and Closure $V$ is compact. Consequently:

If $f \in C_{k}(X)$ and $r>0$, then every essential canonical region of $(f, X, y, r)$ is a k-dimensional manifold whose closure is compact.

If $f \in C_{k}(X)$, then $S(f, X, y)$ is the supremum of the set of all integers $i$ such that there are nonvacuous $k$-dimensional manifolds $V_{1}, V_{2}, \cdots$, $V_{i}$ whose closures are disjoint compact subsets of $X$ and for which $y$ is a stable value of each of the mappings $\left(f \mid V_{1}\right),\left(f \mid V_{2}\right), \cdots,\left(f \mid V_{i}\right)$.

From this alternate characterization of the multiplicity function $S$ we infer that though $S$ was originally defined in terms of the strong "uniform" topology on $C_{k}(X)$, it may equally well be defined in terms of the weaker "compact-open" topology on $C_{k}(X)$ based on the neighborhoods

$$
C_{k}(X) \cap\{g|| g(x)-f(x) \mid<\epsilon \text { for } x \in A\}
$$

of $f$ corresponding to each $\epsilon>0$ and each compact subset $A$ of $X$. 
Convergence in this topology is uniform on every compact subset of $X$.

Whenever $f \in C_{k}(X)$ and $X^{\prime}$ is a locally compact, locally connected subset of $X$, then $\left(f \mid X^{\prime}\right) \in C_{k}\left(X^{\prime}\right)$ and we agree that

$$
S\left(f, X^{\prime}, y\right)=S\left(f \mid X^{\prime}, X^{\prime}, y\right) \quad \text { for } y \in E_{k} \text {. }
$$

We are now ready to state five basic properties of $S$ :

(i) Localizability of $S:$ If $f \in C_{k}(X), X^{\prime}$ is a locally compact, locally connected subset of $X, y \in E_{k}$, and

$$
X \cap\{x \mid f(x)=y\} \subset \text { Interior } X^{\prime},
$$

then

$$
S(f, X, y)=S\left(f, X^{\prime}, y\right) .
$$

(ii) Additivity of $S:$ If $f \in C_{k}(X), y \in E_{k}$, and $P$ is such a disjointed family of open subsets of $X$ that

$$
\bigcup_{W \in P} W=X,
$$

then

$$
S(f, X, y)=\sum_{W \in P} S(f, W, y) .
$$

(iii) Superposition formula for $S$ : If $f \in C_{k}(X), Y$ is a locally compact, locally connected subset of $E_{k}$, range $f \subset Y, g \in C_{k}(Y), z \in E_{k}$, and

$$
Y_{i}=\{y \mid S(f, X, y) \geqq i\} \text { for } i=1,2,3, \cdots,
$$

then

$$
S(g \circ f, X, y) \leqq \sum_{i=1}^{\infty} S\left(g, Y_{i}, z\right) .
$$

Sufficient for equality are the conditions that $X$ be embedded in an orientable $k$-dimensional manifold and that the set

$$
\{y \mid g(y)=z\}
$$

be totally disconnected.

(iv) Compact origin of $S:$ If $f \in C_{n}(X), y \in E_{k}$, and $F$ is the set of all compact, locally connected subsets of $X$, then

$$
S(f, X, y)=\sup _{X^{\prime} \in F} S\left(f, X^{\prime}, y\right) .
$$

(v) Lower semi-continuity of $S: S(f, X, y)$ is lower semi-continuous with respect to $(f, y)$ on the cartesian product $C_{k}(X) \times E_{k}$ with the "com- 
pact-open" topology on $C_{k}(X)$ and the usual topology on $E_{k}$.

The sets $Y_{i}$ in (iii) are open by virtue of $(v)$. The strict inequality may hold in the conclusion of (iii) even if $k=1$ and $X$ and $Y$ are closed intervals.

Combining (v) with Fatou's lemma which states that the lower limit of the integrals of non-negative measurable functions is greater than or equal to the integral of the lower limit of these functions, we find that the $k$-dimensional integralgeometric stable area on $C_{n}(X)$ is lower semi-continuous with respect to the "compact-open" topology on $C_{n}(X)$.

Now suppose that the space $X$ admits finite triangulations. A mapping $g \in C_{n}(X)$ is polyhedral if and only if $X$ has such a finite triangulation $T$ that $g$ maps each simplex of $T$ baricentrically onto some rectilinear simplex of $E_{n}$. The $k$-dimensional area of $g$, defined according to any one of the methods which we have described, equals

$$
\sum_{s \in T_{k}} \mathfrak{H}_{n}^{k}[g(s)]
$$

where $T_{k}$ is the set of all $k$-dimensional simplices of $T$. The Hausdorff measures occurring in this finite sum are computable in elementary fashion by determinants.

The set $P$ of all polyhedral mappings in $C_{n}(X)$ is dense in $C_{n}(X)$. For any mapping $f \in C_{n}(X)$ we have

$$
S\left(p_{n}^{k} \circ R \circ f, X, z\right)=\liminf _{\sigma \rightarrow f, g \in P} N\left(p_{n}^{k} \circ R \circ g, X, z\right)
$$

whenever $R \in G_{n}$ and $z \in E_{k}$; hence the $k$-dimensional integralgeometric stable area of $f$ equals

$$
\beta(n, k)^{-1} \int_{G_{n}} \int_{E_{k}} \liminf _{g \rightarrow f, g \in P} N\left(p_{n}^{k} \circ R \circ g, X, z\right) d \mathcal{L}_{k} z d \phi_{n} R .
$$

True to the tradition of analysis we wonder what happens if we put the "lim inf" ahead of both integral signs. In this way we get the function $L_{k}$ on $C_{n}(X)$ such that

$$
L_{k}(f)=\liminf _{g \rightarrow f, g \in P}(\text { the } k \text {-dimensional area of } g \text { ) }
$$

for any $f \in C_{n}(X)$. The function $L_{k}$ is the $k$-dimensional Lebesgue area on $C_{n}(X)$. We note that, if $X$ is $k$-dimensional, the corresponding upper limit is infinite for all $f \in C_{n}(X)$.

Another way of thinking of the Lebesgue area is the following: The $k$-dimensional area is lower semi-continuous on the dense subset $P$ 
of $C_{n}(X)$, because it may be obtained by confining the lower semicontinuous $k$-dimensional integralgeometric stable area on $C_{n}(X)$ to $P$. Hence the $k$-dimensional area on $P$ can be extended in infinitely many ways to a lower semi-continuous function on $C_{n}(X)$. The numerically largest such extension is the $k$-dimensional Lebesgue area on $C_{n}(X)$.

For any mapping $f \in C_{n}(X)$ the proposition

$$
L_{k}(f)<\infty
$$

has far-reaching analytic and geometric consequences. It implies that $f$ can be uniformly approximated by polyhedral mappings whose areas tend to the Lebesgue area of $f$ and that many properties, such as being quasiconformal, which may be possessed by these polyhedral mappings are then inherited by $f$. For these reasons the Lebesgue area has been used successfully ([R 1$],[D J],[M C S])$ in solving Plateau's problem: to inscribe a surface of least 2-dimensional area in a given simple closed curve in $E_{3}$. It is true that the solution is necessarily a saddle surface for which all the definitions of area described here agree with each other, so that the answer to the problem of Plateau is really independent of the particular definition of area used in stating it precisely. However the Lebesgue area is a powerful tool in proving the existence of solutions of this and other problems in the calculus of variations.

Inasmuch as the $k$-dimensional integralgeometric stable area is less than or equal to $L_{k}(f)$ for all $f \in C_{n}(X)$, it is natural to wonder under what conditions on $k, n$, and $X$ these two areas of $f$ are actually equal for all $f \in C_{n}(X)$. It is known that if $X$ is a $k$-dimensional cell or sphere, then the equality holds in case $1=k \leqq n$ and in case $2=k \leqq n$ $=3$, but fails in case $3 \leqq k \leqq n$ ([R 5$],[\mathrm{F} \mathrm{3}, 6])$.

We now recall the fundamental principle with which we started our whole discussion of areas: Most areas can be defined as integrals of multiplicity functions. Indeed we constructed the Hausdorff $N$ area and the integralgeometric $N$ area according to this scheme, but we varied it somewhat in introducing the integralgeometric stable area, and abandoned it completely in defining the Lebesgue area. This raises the question: Can the Lebesgue area be represented as an integral of a multiplicity function? Our present state of knowledge does not allow a complete answer to this question, but several special cases have been studied thoroughly and in each of these cases the answer is affirmative.

For $k=1$ the Lebesgue area equals the Hausdorff $N$ area.

For $k=n$ the problem was solved in [F 6], though with the unneces- 
sary restriction that the manifold in which $X$ is embedded be orientable, by the discovery of such a new multiplicity function $M$ that the Lebesgue area equals the Hausdorff $M$ area. We shall presently describe $M$, without the assumption of orientability.

In the later parts of this paper we shall answer the question for the case in which $k=2, n=3$, and $X$ is a 2-cell.

Our characterization of the stable multiplicity $S$ in terms of the Cech cohomology theory suggests the following definition of the new function $M$ : Suppose $X$ is a locally compact, locally connected, separable subset of a $k$-dimensional manifold (not necessarily admitting finite triangulations), $f \in C_{k}(X)$, and $y \in E_{k}$. For $r>0$ and each canonical region $V$ of $(f, X, y, r)$ we consider the induced homomorphism

$$
f^{*}: H^{k}\left(E_{k}, E_{k} \cap\{z|| z-y \mid \geqq r\}\right) \rightarrow H^{k} \text { (Closure } V \text {, Boundary } V \text { ) }
$$

of the $k$-dimensional Cech cohomology group with integer coefficients and based on locally finite coverings. The first group is infinite cyclic. According to the three possible isomorphism types of the second group we define the non-negative integer

$$
D(f, r, V)
$$

in three alternate steps:

(i) If $V$ is an orientable $k$-dimensional manifold whose closure is compact, then the second group is infinite cyclic, $f^{*}$ maps a generator of the first group onto an integral multiple of a generator of the second group, and $D(f, r, V)$ is the absolute value of the multiplier.

(ii) If $V$ is a nonorientable $k$-dimensional manifold whose closure is compact, the second group is cyclic of order two and $D(f, r, V)$ equals 0 or 1 depending on whether $f^{*}$ is trivial or nontrivial.

(iii) Otherwise the second group is trivial and $D(f, r, V)=0$. Letting $F(r)$ be the set of all canonical regions of $(f, X, y, r)$ whenever $r>0$, we see that

$$
\sum_{v \in F_{(r)}} D(f, r, V)
$$

does not decrease with $r$. The limit, as $r \rightarrow 0+$, of this sum is the multiplicity

$$
M(f, X, y) \text {. }
$$

Proceeding as in [F 6] we find that the function $M$ is connected with the Lebesgue area by the following theorem:

If $X$ admits finite triangulations and $f \in C_{k}(X)$, then 


$$
L_{k}(f)=\int_{E_{k}} M(f, X, y) d \mathcal{L}_{k} y .
$$

The triangulation hypothesis can be removed by a suitable natural extension of the concept of Lebesgue area (see \$6).

The functions $S$ and $M$ are related by the inequality

$$
S(f, X, y) \leqq M(f, X, y) \text { for } f \in C_{k}(X), y \in E_{k},
$$

which can be strict for a set of points $y$ with positive $\mathcal{L}_{k}$ measure. However these two multiplicities vanish simultaneously and are simultaneously infinite. Furthermore the five listed basic properties (localizability, additivity, superposition formula, compact origin, lower semi-continuity) of $S$ hold also for $M$.

Returning to the general case in which $k \leqq n$ we conclude this section by discussing two properties of $L_{k}$ on $C_{n}(X)$ which will be of later use:

(1) If $X$ is finitely triangulable, $f \in C_{n}(X)$, and $G$ is the set of all components of $X$, then

$$
L_{k}(f)=\sum_{Z \in G} L_{k}(f \mid Z) .
$$

(2) If $X$ is finitely triangulable, $f \in C_{n}(X)$, and $Z$ is a finitely triangulable subset of $X$, then

$$
L_{k}(f) \geqq L_{k}(f \mid Z) .
$$

The first of these two propositions is obvious, but the second seems worthy of further discussion.

Suppose $\epsilon>0$ and $g \in C_{n}(X)$ is a polyhedral mapping, with a corresponding finite triangulation $T$ of $X$, such that

$$
\begin{aligned}
& |g(x)-f(x)|<\epsilon \text { for } x \in X, \\
& L_{k}(g) \leqq L_{k}(f)+\epsilon .
\end{aligned}
$$

Letting $\rho$ be any distance function metrizing the compact space $X$, we next choose $\delta>0$ so that

$$
\left|g(x)-g\left(x^{\prime}\right)\right|<\epsilon \text { whenever } \rho\left(x, x^{\prime}\right)<2 \delta .
$$

From $T$ we obtain by successive barycentric subdivisions a finite triangulation $T^{\prime}$ of $X$ such that the star of each vertex of $T^{\prime}$ has a diameter less than $\delta$. Then the mapping $g$ is polyhedral with respect to $T^{\prime}$. Let $P$ be the set of all $k$ simplices of $T^{\prime}$ and let

$$
Q=P \cap\{s \mid s \subset Z\} \text {. }
$$


We next choose a sufficiently fine finite triangulation $U$ of $Z$ and a function $q$ on $Q$ to the set of all $k$ simplices of $U$ such that

$$
q(s) \subset s \text { for } s \in Q \text {. }
$$

Applying the modification procedure of [F 6] to the identity map of $Z$ into $X$ we obtain a map $u$ of $Z$ into $X$ with the following properties:

$$
\begin{aligned}
& u \text { maps } q(s) \text { barycentrally onto } s \text { for } s \in Q, \\
& u[s-q(s)] \subset \text { Boundary } s \text { for } s \in Q, \\
& u(s \cap Z) \subset \text { Boundary } s \text { for } s \in P-Q .
\end{aligned}
$$

It follows that

$$
\rho[u(x), x]<\delta \text { for } x \in Z .
$$

From $U$ and $u$ we obtain by successive barycentric subdivisions and simplicial approximation a finite triangulation $U^{\prime}$ of $Z$ and a map $u^{\prime}$ of $Z$ into $X$ such that:

$u^{\prime}$ is simplicial with respect to $U^{\prime}$ and $T^{\prime}$,

$$
\begin{aligned}
& \rho\left[u^{\prime}(x), u(x)\right]<\delta \text { for } x \in Z, \\
& u^{\prime}|q(s)=u| q(s) \text { for } s \in Q, \\
& u \text { maps } Z-\bigcup_{s \in Q} q(s) \text { into the } k-1 \text { skeleton of } T^{\prime} .
\end{aligned}
$$

We conclude that the mapping $\left(g \circ u^{\prime}\right) \in C_{n}(Z)$ is polyhedral with

$$
\begin{gathered}
\rho\left[u^{\prime}(x), x\right]<2 \delta, \quad\left|\left(g \circ u^{\prime}\right)(x)-g(x)\right|<\epsilon, \\
\left|\left(g \circ u^{\prime}\right) x-(f \mid Z)(x)\right|<2 \epsilon \text { for } x \in Z
\end{gathered}
$$

and that

$$
L_{k}\left(g \circ u^{\prime}\right)=\sum_{s \in Q} \mathcal{H}_{n}^{k}[g(s)] \leqq L_{k}(g) \leqq L_{k}(f)+\epsilon .
$$

This concludes the proof of the Proposition (2).

6. Integration over the middle space. The results of the preceding section suggest that among all the open subsets of $X$ the canonical regions associated with a mapping $f \in C_{n}(X)$ are most important in computing those multiplicities of $f$ which are connected with the Lebesgue area of $f$. For $x \in X$ and $r>0$ we accordingly consider the neighborhood

$$
\Delta_{f}(x, r)
$$

of $x$, which is defined as the canonical region $U$ of $(f, X, f(x), r)$ 
such that $x \in U$.

These neighborhoods do not always form a basis for the topology of $X$, for it may happen that

$$
\Delta_{f}(x, r)=\Delta_{f}\left(x^{\prime} r\right) \text { for all } r>0
$$

even though $x$ and $x^{\prime}$ are distinct points of $X$. Therefore we consider the equivalence relation

$$
(X \times X) \cap\left\{\left(x, x^{\prime}\right) \mid \Delta_{f}(x, r)=\Delta_{f}\left(x^{\prime}, r\right) \text { for all } r>0\right\}
$$

and the corresponding partition

$\mathcal{M}_{f}$

of $X$ into equivalence classes, called the middle space of $f$. Each $x \in X$ is an element of the equivalence class

$$
m_{f}(x)=\bigcap_{r>0} \Delta_{f}(x, r) \in \mathscr{X}_{f} .
$$

The function $m_{f}$ has domain $X$ and range $\mathscr{X}_{f}$.

Assuming that $X$ is connected we metrize the set $\mathcal{X}_{f}$ (as in [B 2]) by means of the distance function $d_{f}$ as follows: If $A \in \mathcal{X}_{f}$ and $B \in \mathcal{X}_{f}$, then

$$
d_{f}(A, B)=\inf _{W \in F} \operatorname{diameter} f(W)
$$

where $F$ is the set of all those compact connected subsets of $X$ which meet both $A$ and $B$.

The connectedness of $X$ is essential only in proving that $d_{f}(A, B)$ $<\infty$ for all $A, B \in \mathcal{X}_{f}$. If we wanted to drop the assumption that $X$ is connected, we could replace $d_{f}(A, B)$ by $d_{f}(A, B) /\left[1+d_{f}(A, B)\right]$ in case $d_{f}(A, B)<\infty$ and by 1 in case $d_{f}(A, B)=\infty$. All subsequent results would remain substantially unchanged.

The metric $d_{f}$ induces such a topology on $\mathscr{X}_{f}$ that the function $m_{f}$ is continuous, the $m_{f}$ counterimage of every connected open subset of $\mathscr{X}_{f}$ is a connected subset of $X$, and the space $\mathcal{X}_{f}$ is locally connected.

If $A \in \mathcal{X}_{f}$ and $r>0$, then $\Delta_{f}(x, r)$ is the same for all $x \in A$. It will be denoted by

$$
\Delta_{f}(A, r) .
$$

The elements of $\mathscr{x}_{f}$ are closed subsets of $X$ but need be neither compact nor connected. If $A \in \mathcal{X}_{f}$, then $A$ is a compact subset of $X$ if and only if the sets $\Delta_{f}(A, r)$ have compact closures for all suffi- 
ciently small positive numbers $r$. Every compact element of $\mathcal{X}_{f}$ is connected. The set of all compact elements of $\mathscr{X}_{f}$ is an open locally compact subset of $\mathscr{X}_{f}$. The whole space $\mathscr{X}_{f}$ need not be locally compact.

If $X$ is compact, then the elements of $\mathscr{X}_{f}$ are the maximal continua of constancy of $f$ and the mapping $m_{f}$ is monotone. However, if $X$ is not compact, then $\mathscr{X}_{f}$ and $m_{f}$ need not have these properties.

In verifying these properties of $\mathcal{X}_{f}, m_{f}$, and $d_{f}$ it is helpful to note that

$$
\begin{gathered}
\left\{A \mid d_{f}\left[A, m_{f}(x)\right]<r\right\} \subset m_{f}\left[\Delta_{f}(x, r)\right] \subset\left\{A \mid d_{f}\left[A, m_{f}(x)\right]<2 r\right\}, \\
\left\{z \mid m_{f}(z) \in m_{f}\left[\Delta_{f}(x, r)\right]\right\}=\Delta_{f}(x, r) \quad \text { for } x \in X, r>0 .
\end{gathered}
$$

Since $f$ is constant on each equivalence class in $\mathcal{X}_{f}$, there is a unique function $l_{f}$ on $\mathscr{X}_{f}$ to $E_{n}$ such that

$$
f=l_{f} \circ m_{f} .
$$

$l_{f}$ is continuous; in fact it satisfies the Lipschitz condition

$$
\left|l_{f}(A)-l_{f}(B)\right| \leqq d_{f}(A, B) \text { for } A, B \in \mathcal{X}_{f} .
$$

If $Y$ is any subset of $E_{n}$ and $Z$ is any component of the $l_{f}$ counterimage of $Y$, then the diameter of $Z$ is not greater than the diameter of $Y$. It follows that the $l_{f}$ counterimage of each point of $E_{n}$ is totally disconnected, so that $l_{f}$ is a light mapping.

Recalling the manner in which $\mathfrak{K}_{n}^{\boldsymbol{k}}$ was defined in terms of the metric of $E_{n}$ we analogously define the k-dimensional Hausdorff measure

$$
H_{f}^{k}
$$

over $\mathcal{X}_{f}$ in terms of the metric $d_{f}$. The Lipschitz condition satisfied by $l_{f}$ implies that

$$
H_{f}^{k}(Q) \geqq \mathcal{F}_{n}^{k}\left[l_{f}(Q)\right] \quad \text { whenever } Q \subset \mathscr{M}_{f}
$$

It follows that

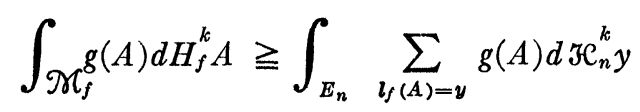

for every non-negative analytically measurable function $g$ on $\mathscr{X}_{f}$.

To prove this we observe that the set of all those non-negative analytically measurable functions $g$ for which the above inequality holds is closed to addition, to multiplication by positive numbers, and to monotone convergence. Hence it is sufficient to prove that 
this set contains all characteristic functions of analytic subsets of $\mathcal{X}_{f}$. However, if $g$ is the characteristic function of the analytic set $Q \subset \mathcal{X}_{f}$, then the inequality reduces to the formula

$$
H_{f}^{k}(Q) \geqq \int_{E_{n}} N\left(l_{f}, Q, y\right) d \mathcal{K}_{n}^{k} y
$$

which can be proved by the method of $[F 1,4.4]$.

If $Q \subset \mathcal{X}_{f}$ and $H_{f}^{k}(Q)<\infty$, then

$$
2^{-k} \leqq \limsup _{r \rightarrow 0+} \frac{H_{f}^{k}\left(Q \cap m_{f}\left[\Delta_{f}(A, r)\right]\right)}{\alpha(k) r^{k}} \leqq 1
$$

for $H_{f}^{k}$ almost all $A$ in $Q$. If, in addition, the set $Q$ is $H_{f}^{k}$ measurable, then

$$
\lim _{r \rightarrow 0+} \frac{H_{f}^{k}\left(Q \cap m_{f}\left[\Delta_{f}(A, r)\right]\right)}{\alpha(k) r^{k}}=0
$$

for $H_{f}^{k}$ almost all $A$ in $\mathscr{X}_{f}-Q$.

These density properties of $H_{f}^{k}$ can be proved just like the corresponding properties of $\mathfrak{F}_{n}^{k}$ established in [F 4].

An arbitrary subset $V$ of $X$ may fail to be finitely triangulable and the $k$-dimensional Lebesgue area of the mapping $f \mid V$ may not be well defined. We therefore define the substitute

$$
\lambda_{k}(f, V)
$$

as the supremum of $L_{k}(f \mid W)$ for all those subsets $W$ of $V$ which possess finite triangulations. It is clear that

$$
\lambda_{k}(f, V)=L_{k}(f \mid V) \text { for every finitely triangulable } V \subset X,
$$

$\lambda_{k}(f, V) \geqq \sum_{i=1}^{\infty} \lambda_{k}\left(f, V_{i}\right)$ whenever $V_{1}, V_{2}, V_{3}, \cdots$ are disjoint subsets of $V \subset X$.

If $A \in \mathscr{X}_{f}$ and $r>0$, then

$$
f\left[\Delta_{f}(A, r)\right] \subset K\left[l_{f}(A), r\right],
$$

and it is natural to compare $\lambda_{k}\left[f, \Delta_{f}(A, r)\right]$ with $\alpha(k) r^{k}$, the $\mathcal{L}_{k}$ measure of any orthogonal projection of $K\left[l_{f}(A), r\right]$ into $E_{k}$. This leads us to consider the upper and lower limits

$$
L_{k}^{*}(f, A)=\limsup _{r \rightarrow 0+} \frac{\lambda_{k}\left[f, \Delta_{f}(A, r)\right]}{\alpha(k) r^{k}},
$$




$$
L_{* k}(f, A)=\liminf _{r \rightarrow 0+} \frac{\lambda_{k}\left[f, \Delta_{f}(A, r)\right]}{\alpha(k) r^{k}},
$$

which we think of as the upper and lower densities of the $k$-dimensional Lebesgue area of $f$ at $A$. Using integration with respect to $H_{f}^{k}$ we shall establish formulae which allow us to regard these densities as derivatives of the Lebesgue area. Furthermore the densities are related to multiplicity functions connected with the Lebesgue area.

We note that $\lambda_{k}\left[f, \Delta_{f}(A, r)\right]$ is lower semi-continuous with respect to $(A, r)$ on the cartesian product space $\mathcal{X}_{f} \times\{r \mid r>0\}$. It follows that $L_{k}^{*}(f, A)$ and $L_{* k}(f, A)$ are analytically measurable with respect to $A$ on $\mathscr{M}_{f}$.

LEMmA 6.1. If $f \in C_{n}(X), V$ is an open subset of $\mathcal{X C}_{f}, W \subset V, t>0$, and

$$
L_{k}^{*}(f, A)>t \quad \text { for } A \in W \text {, }
$$

then

$$
\lambda_{k}\left(f,\left\{x \mid m_{f}(x) \in V\right\}\right) \geqq t \cdot H_{f}^{k}(W) .
$$

Proof. We may assume that

$$
\lambda_{k}\left(f,\left\{x \mid m_{f}(x) \in V\right\}\right)<\infty .
$$

Let $\epsilon>0$. Defining

$$
\begin{gathered}
u(A, r)=\text { Closure } m_{f}\left[\Delta_{f}(A, r)\right] \text { for } A \in \mathcal{X}_{f} \text { and } r>0, \\
F=\left\{u(A, r) \mid A \in \mathcal{X}_{f}, 0<r<\epsilon / 10, u(A, r) \subset V\right. \text { and } \\
\left.\lambda_{k}\left[f, \Delta_{f}(A, r)\right]>t \alpha(k) r^{k}\right\}, \\
\widehat{Z}=\left\{A \mid d_{f}(A, B) \leqq 2 \operatorname{diam} Z \text { for some } B \in Z\right\} \text { for } Z \subset \mathcal{X}_{f},
\end{gathered}
$$

we note that $F$ covers $W$ in the sense of Vitali and apply a covering theorem of A. P. Morse [M, 3.10] to obtain such a disjointed subfamily $G$ of $F$ that

$$
W \subset \underset{z \in G}{\bigcup} Z \cup \underset{z \in \in_{H}}{\bigcup} \widehat{Z}
$$

whenever $H \subset G$ and $G-H$ is finite. For each $Z \in G$ we choose $A_{z}$ and $r_{Z}$ so that

$$
\begin{gathered}
Z=u\left(A_{Z}, r_{Z}\right), \quad A_{Z} \in \mathcal{P} c_{f}, \quad 0<r_{Z}<\epsilon / 10, \\
\lambda_{k}\left[f, \Delta_{f}\left(A_{Z}, r_{Z}\right)\right]>t \alpha(k)\left(r_{Z}\right)^{k}
\end{gathered}
$$


and observe that

diameter $Z \leqq 2 r_{Z}<\epsilon$, diameter $\widehat{Z} \leqq 5$ diameter $Z \leqq 10 r_{Z}<\epsilon$.

If $Z$ and $Z^{\prime}$ are distinct elements of $G$, then $\Delta_{f}\left(A_{Z}, r_{Z}\right)$ and $\Delta_{f}\left(A_{Z^{\prime}}, r_{Z^{\prime}}\right)$ are disjoint nonvacuous open subsets of $X$. It follows that $G$ is countable.

If $H \subset G$ and $G-H$ is finite, then

$$
G \cup\{\widehat{Z} \mid Z \in H\}
$$

is a countable covering of $W$ by sets whose diameter is less than $\epsilon$ and

$$
\begin{aligned}
\sum_{Z \in G} 2^{-k} \alpha(k)(\text { diameter } Z)^{k}+\sum_{Z \in H} 2^{-k} \alpha(k)(\text { diameter } \widehat{Z})^{k} \\
\quad \leqq \sum_{Z \in G} \alpha(k)\left(r_{Z}\right)^{k}+5^{k} \sum_{Z \in H} \alpha(k)\left(r_{Z}\right)^{k} \\
\leqq t^{-1}\left(\sum_{Z \in G} \lambda_{k}\left[f, \Delta_{f}\left(A_{Z}, r_{Z}\right)\right]+\sum_{Z \in H} \lambda_{k}\left[f, \Delta_{f}\left(A_{Z}, r_{Z}\right)\right]\right) .
\end{aligned}
$$

Inasmuch as

$$
\sum_{Z \in G} \lambda_{k}\left[f, \Delta_{f},\left(A_{Z}, r_{Z}\right)\right] \leqq \lambda_{k}\left(f,\left\{x \mid m_{f}(x) \in V\right\}\right),
$$

we can choose $H$ so that

$$
\sum_{z \in H} \lambda_{k}\left[f, \Delta_{f}\left(A_{z}, r_{z}\right)\right]
$$

is arbitrarily small. Then the last member of our string of inequalities exceeds

$$
t^{-1} \lambda_{k}\left(f,\left\{x \mid m_{f}(x) \in V\right\}\right)
$$

by arbitrarily little.

Letting $\epsilon$ approach 0 we conclude that

$$
H_{f}^{k}(W) \leqq t^{-1} \lambda_{k}(f,\{x \mid m(x) \in V\}) .
$$

THEOREM 6.2. If $f \in C_{n}(X)$ and $V$ is an open subset of $\mathcal{X}_{f}$, then

$$
\lambda_{k}\left(f,\left\{x \mid m_{f}(x) \in V\right\}\right) \geqq \int_{V} L_{k}^{*}(f, A) d H_{f}^{k} A .
$$

Proof. We may assume that

$$
\lambda_{k}\left(f,\left\{x \mid m_{f}(x) \in V\right\}\right)<\infty .
$$

Let $1<t<\infty$. For each integer $i$ we define 


$$
U_{i}=V \cap\left\{A \mid t^{i}<L_{k}^{*}(f, A) \leqq t^{i+1}\right\},
$$

infer from the preceding lemma that

$$
H_{f}^{k}\left(U_{i}\right) \leqq t^{-i} \lambda_{k}\left[f,\left\{x \mid m_{f}(x) \in V\right\}\right]<\infty,
$$

and choose a closed subset $W_{i}$ of $U_{i}$ such that

$$
H_{f}^{k}\left(U_{i}\right) \leqq t H_{f}^{k}\left(W_{i}\right) .
$$

Since the sets $W_{i}$ are disjoint closed subsets of $V$, we can choose disjoint open subsets $V_{i}$ of $V$ such that $W_{i} \subset V_{i}$ for every positive integer $i$.

Applying the preceding lemma again, we conclude that

$$
\begin{aligned}
\int_{V} L_{k}^{*}(f, A) d H_{f}^{k} A & =\sum_{i=-\infty}^{\infty} \int_{U_{i}} L_{k}^{*}(f, A) d H_{f}^{k} A \\
& \leqq \sum_{i=-\infty}^{\infty} t^{i+1} \cdot H_{f}^{k}\left(U_{i}\right) \leqq t^{2} \sum_{i=-\infty}^{\infty} t^{i} \cdot H_{f}^{k}\left(W_{i}\right) \\
& \leqq t^{2} \sum_{i=-\infty}^{\infty} \lambda_{k}\left(f,\left\{x \mid m_{f}(x) \in V_{i}\right\}\right) \\
& \leqq t^{2} \lambda_{k}\left(f,\left\{x \mid m_{f}(x) \in V\right\}\right)
\end{aligned}
$$

and let $t$ approach 1 to complete the proof.

The preceding theorem is complemented by the inequality

$$
\int_{V} L_{k}^{*}(f, A) d H_{f}^{k} A \geqq \int_{E_{n}} \sum_{A \in V, l_{f}(A)=y} L_{k}^{*}(f, A) d \mathcal{C}_{n}^{k} y
$$

which is valid whenever $f \in C_{n}(X)$ and $V$ is an analytic subset of $\mathscr{X}_{f}$.

It is natural to ask:

Does equality hold in these inequalities?

Is $L_{\mathbf{k}}^{*}(f, A)=L_{* k}(f, A)$ for $H_{f}^{k}$ almost all $A$ in $\mathscr{X l}_{f}$ ?

No counterexamples are known. Moreover we shall prove the affirmative answers for some special cases. The problem is trivial for $k=1$ $\leqq n$. The case in which $k=n$, the case in which $S$ is a finitely connected subset of $E_{2}$ and $n \geqq 2$, and in particular the case in which $X$ is a 2 -cell and $n=3$ will be discussed in detail. Complete answers will be obtained in the first and last of these three cases. In general the solution of these problems seems to depend on the answer to the following question:

What geometric properties of a point $A \in \mathcal{X}_{f}$ and which topological and metric invariants of the mappings $f \mid \Delta_{f}(a, r)$, for $r>0$, are significant 
for the densities $L_{\mathbf{k}}^{*}(f, A)$ and $L_{* k}(f, A)$ ?

One such property of $A$ is that of being $i$-fold essential in dimension $k$, which we shall define later in this section. In some special cases the densities can be completely described in terms of this property. However the geometry underlying the behavior of the densities is not yet fully understood in the general case.

In the remainder of this section we compile the fragmentary results known to us for the general case in which $k \leqq n$ as well as the complete results for the case in which $k=n$. The case in which $X$ is a finitely connected compact subset of $E_{2}$ is taken up in the later sections.

TheOREM 6.3. If $f \in C_{n}(X), V$ is an open subset of $\mathcal{X}_{f}$,

$$
\lambda_{k}\left(f,\left\{x \mid m_{f}(x) \in V\right\}\right)=\int_{V} L_{k}^{*}(f, A) d H_{f}^{k} A<\infty,
$$

and $V^{\prime}$ is an open subset of $V$, then

$$
\lambda_{k}\left(f,\left\{x \mid m_{f}(x) \in V^{\prime}\right\}\right)=\int_{V^{\prime}} L_{k}^{*}(f, A) d H_{f}^{k} A .
$$

Proof. Otherwise there is a finitely triangulable set $W$ such that

$$
W \subset\left\{x \mid m_{f}(x) \in V^{\prime}\right\}, L_{k}(f \mid W)>\int_{V^{\prime}} L_{k}^{*}(f, A) d H_{f}^{k} A .
$$

Then $W$ and $m_{f}(W)$ are compact, $V-m_{f}(W)$ is open, $W$ and $\left\{x \mid m_{f}(x) \in V-m_{f}(W)\right\}$ are disjoint subsets of $\left\{x \mid m_{f}(x) \in V\right\}$, and $m_{f}(W) \subset V^{\prime}$. It follows that

$$
\begin{aligned}
\lambda_{k}\left(f,\left\{x \mid m_{f}(x) \in V\right\}\right) & \geqq L_{k}(f \mid W)+\lambda_{k}\left(f,\left\{x \mid m_{f}(x) \in V-m_{f}(W)\right\}\right) \\
& >\int_{V^{\prime}} L_{k}^{*}(f, A) d H_{f}^{k} A+\int_{V-m_{f}(W)} L_{k}^{*}(f, A) d H_{f}^{k} A \\
& \geqq \int_{V} L_{k}^{*}(f, A) d H_{f}^{k} A=\lambda_{k}\left(f,\left\{x \mid m_{f}(x) \in V\right\}\right),
\end{aligned}
$$

which is false.

Lemma 6.4. If $f \in C_{k}(X)$ and $U$ is an open subset of $X$, then

$$
\lambda_{k}(f, U)=\int_{E_{k}} M(f, U, y) d \mathcal{L}_{k} y .
$$

PROoF. If $W$ is any finitely triangulable subset of $U$, then 


$$
\begin{gathered}
L_{k}(f \mid W)=\int_{E_{k}} M(f, W, y) d \mathcal{L}_{k} y, \\
M(f, W, y) \leqq M(f, U, y) \text { for } y \in E_{k} .
\end{gathered}
$$

It follows that

$$
\lambda_{k}(f, U) \leqq \int_{E_{k}} M(f, U, y) d \mathcal{L}_{k} y .
$$

On the other hand $U$ has finitely triangulable subsets $W_{1} \subset W_{2}$ $C W_{3} \subset \cdots$ with the property that each point of $U$ which has a $k$ cell neighborhood in $U$ is an interior point of $W_{i}$ for some positive integer $i$. We infer that

$$
\begin{gathered}
M\left(f, W_{i}, y\right) \leqq M\left(f, W_{i+1}, y\right) \text { for } y \in E_{k} \text { and } i=1,2,3, \cdots, \\
M(f, U, y)=\lim _{i \rightarrow \infty} M\left(f, W_{i}, y\right) \text { for } y \in E_{k}, \\
\int_{E_{k}} M(f, U, y) d \mathcal{L}_{k} y=\lim _{i \rightarrow \infty} \int_{E_{k}} M\left(f, W_{i}, y\right) d \mathcal{L}_{k} y \\
=\lim _{i \rightarrow \infty} L_{k}\left(f \mid W_{i}\right) \leqq \lambda_{k}(f, U) .
\end{gathered}
$$

Theorem 6.5. If $f \in C_{n}(X), V$ is an open subset of $\mathcal{H}_{f}$,

$$
\begin{gathered}
\lambda_{k}\left(f,\left\{x \mid m_{f}(x) \in V\right\}\right)=\int_{V} L_{k}^{*}(f, A) d H_{f}^{k} A<\infty, \\
P=V \cap\left\{A \mid L_{k}^{*}(f, A)>0\right\}, \quad R \in G_{n}, \\
Z=\left\{z \mid S\left(p_{n}^{k} \circ R \circ f,\left\{x \mid m_{f}(x) \in V\right\}, z\right)>0\right\},
\end{gathered}
$$

then

$$
\mathcal{L}_{k}\left[Z-\left(p_{n}^{k} \circ R \circ l_{f}\right)(P)\right]=0 .
$$

Proof. Otherwise there exist a closed set $F$ and open sets $U_{1} \supset U_{2}$ $\supset U_{3} \supset \cdots$ contained in $E_{k}$ such that

$$
\mathcal{L}_{k}(F)>0 \text { and } \bigcap_{i=1}^{\infty} U_{i}=F \subset Z-\left(p_{n}^{k} \circ R \circ l_{f}\right)(P) .
$$

Defining

$$
\begin{aligned}
& F^{\prime}=V \cap\left\{A \mid\left(p_{n}^{k} \circ R \circ l_{f}\right)(A) \in F\right\}, \\
& U_{i}^{\prime}=V \cap\left\{A \mid\left(p_{n}^{k} \circ R \circ l_{f}\right)(A) \in U_{i}\right\} \text { for } i=1,2,3, \cdots,
\end{aligned}
$$

we infer that 


$$
V \supset U_{1}^{\prime} \supset U_{2}^{\prime} \supset U_{3}^{\prime} \supset \cdots \text { and } \bigcap_{i=1}^{\infty} U_{i}^{\prime}=F^{\prime} \subset V-P \text {, }
$$

whence

$$
\lim _{i \rightarrow \infty} \int_{U_{1}^{\prime}} L_{k}^{*}(f, A) d H_{f}^{k} A=\int_{F^{\prime}} L_{k}^{*}(f, A) d H_{f}^{k} A=0
$$

and we choose a positive integer $i$ for which

$$
\int_{U_{1}^{\prime}} L_{k}^{*}(f, A) d H_{f}^{k} A<\mathcal{L}_{k}(F)
$$

However

$$
\begin{aligned}
\mathcal{L}_{k}(F) & \leqq \int_{F} S\left(p_{n}^{k} \circ R \circ f,\left\{x \mid m_{f}(x) \in V\right\}, z\right) d \mathcal{L}_{k} z \\
& \leqq \int_{U_{i}} M\left(p_{n}^{k} \circ R \circ f,\left\{x \mid m_{f}(x) \in V\right\}, z\right) d \mathcal{L}_{k} z \\
& =\int_{U_{i}} M\left(p_{n}^{k} \circ R \circ f,\left\{x \mid m_{f}(x) \in U_{i}^{\prime}\right\}, z\right) d \mathcal{L}_{k} z \\
& =\int_{E_{k}} M\left(p_{n}^{k} \circ R \circ f,\left\{x \mid m_{f}(x) \in U_{i}^{\prime}\right\}, z\right) d \mathcal{L}_{k} z \\
& =\lambda_{k}\left(p_{n}^{k} \circ R \circ f,\left\{x \mid m_{f}(x) \in U_{i}^{\prime}\right\}\right) \leqq \lambda_{k}\left(f,\left\{x \mid m_{f}(x) \in U_{i}^{\prime}\right\}\right) \\
& =\int_{U_{1}^{\prime}} L_{k}^{*}(f, A) d H_{f}^{k} A .
\end{aligned}
$$

Theorem 6.6. If $f \in C_{n}(X), V$ is an open subset of $\mathscr{X}_{f}$,

$$
\lambda_{k}\left(f,\left\{x \mid m_{f}(x) \in V\right\}\right)=\int_{V} L_{k}^{*}(f, A) d H_{f}^{k} A<\infty,
$$

and $R \in G_{n}$, then

$$
\begin{aligned}
S\left(p_{n}^{k} \circ R \circ f,\left\{x \mid m_{f}(x)\right.\right. & \in V\}, z) \\
& \leqq N\left(p_{n}^{k} \circ R \circ l_{f}, V \cap\left\{A \mid L_{k}^{*}(f, A)>0\right\}, z\right)
\end{aligned}
$$

for $\mathcal{L}_{k}$ almost all $\boldsymbol{z}$ in $E_{k}$.

Proof. We choose a countable basis $B$ for the topology of $E_{k}$, define

$$
F_{U}=\left\{W \mid W \text { is a component of } V \cap\left\{A \mid\left(p_{n}^{k} \circ R \circ l_{f}\right)(A) \in U\right\}\right\}
$$


for each $U \in B$ and let

$$
Z_{W}=\left\{z \mid S\left(p_{n}^{k} \circ R \circ f,\left\{x \mid m_{f}(x) \in W\right\}, z\right)>0\right\}
$$

whenever $W \in F_{U}$ for some $U \in B$. Then

$$
\bigcup_{U} \in_{B} F_{U} \text { is countable }
$$

and we infer from Theorem 6.5 that

$$
\mathcal{L}_{k}\left(\bigcup_{U} \in_{B} \bigcup_{W}\left[Z_{W}-\left(p_{n}^{k} \circ R \circ l_{f}\right)(W \cap P)\right]\right)=0
$$

where

$$
P=\left\{A \mid L_{k}^{*}(f, A)>0\right\} .
$$

Now suppose

$$
z \in E_{k}-\bigcup_{U \in \in_{B}} \bigcup_{W}\left[Z_{W}-\left(p_{n}^{k} \circ R \circ l_{f}\right)(W \cap P)\right]
$$

$m$ is a positive integer, and

$$
S\left(p_{n}^{k} \circ R \circ f,\left\{x \mid m_{f}(x) \in V\right\}, z\right) \geqq m .
$$

Then there exists a $U \in B$ such that the set

$$
G=F_{U} \cap\left\{W \mid z \in Z_{W}\right\}
$$

has at least $m$ elements. Recalling our choice of $z$ we find that

$$
z \in\left(p_{n}^{k} \circ R \circ l_{f}\right)(W \cap P) \text { for } W \in G
$$

and use the disjointedness of $G$ to conclude that

$$
N\left(p_{n}^{k} \circ R \circ l_{f}, V \cap P, z\right) \geqq m .
$$

The proof is complete.

We say that

$A$ is $i$-fold essential for $f$ in dimension $k$ if and only if $f \in C_{n}(X)$, $A \in \mathscr{X}_{f}, i$ is an integer, and there exists an $R \in G_{n}$ such that

$$
\lim _{r \rightarrow 0+} \frac{\mathcal{L}_{k}\left(\left\{z \mid M\left(p_{n}^{k} \circ R \circ f, \Delta_{f}(A, r), z\right) \geqq i\right\}\right)}{\alpha(k) r^{k}}=1 .
$$

We refer to $p_{n}^{k} \circ R$ as an $i$-fold essential projection for $f$ at $A$ in dimension $k$. 
Furthermore we define

$\mathcal{E}_{k}(f, A)=\sup \{i \mid A$ is $i$-fold essential for $f$ in dimension $k\}$.

We note that $M\left(p_{n}^{k} \circ R \circ f, \Delta_{f}(A, r), z\right)$ is lower semi-continuous with respect to $(A, R, r, z)$ on the cartesian product space $\mathcal{X}_{f} \times G_{n}$ $\times\{r \mid r>0\} \times E_{k}$, and that

$$
\mathcal{L}_{k}\left(\left\{z \mid M\left(p_{n}^{k} \circ R \circ f, \Delta_{f}(A, r), z\right) \geqq i\right\}\right)
$$

is lower semi-continuous with respect to $(A, R, r)$ on the cartesian product space $\mathcal{H}_{f} \times G_{n} \times\{r \mid r>0\}$ for each integer $i$. It follows that $\mathcal{E}_{k}(f, A)$ is analytically measurable with respect to $A$ on $\mathcal{H}_{f}$.

Theorem 6.7. $L_{* k}(f, A) \geqq \mathcal{E}_{k}(f, A)$ for $f \in C_{n}(X)$ and $A \in \mathcal{X}_{f}$.

Proof. If $R \in G_{n}$, then

$$
\begin{aligned}
\lambda_{k}\left[f, \Delta_{f}(A, r)\right] & \geqq \lambda_{k}\left[p_{n}^{k} \circ R \circ f, \Delta_{f}(A, r)\right] \\
& =\int_{E_{k}} M\left[p_{n}^{k} \circ R \circ f, \Delta_{f}(A, r)\right] d \mathcal{L}_{k^{z}} \\
& \geqq i \mathcal{L}_{k}\left(\left\{z \mid M\left(p_{n}^{k} \circ R \circ f, \Delta_{f}(A, r), z\right) \geqq i\right\}\right) .
\end{aligned}
$$

TheOREM 6.8. If $p_{n}^{k} \circ R$ is an $i$-fold essential projection for $f$ at $A$ in dimension $k, \epsilon>0$, and

$$
T(\boldsymbol{r})=\Delta_{f}(A, r) \cap\left\{x \mid f(x) \in \diamond_{n}^{k}\left[R, \epsilon, l_{f}(A)\right]\right\} \quad \text { for } r>0,
$$

then

$$
\lim _{r \rightarrow 0+} \frac{\mathcal{L}_{k}\left(\left\{z \mid M\left(p_{n}^{k} \circ R \circ f, T(r), z\right) \geqq i\right\}\right)}{\alpha(k) r^{k}}=1 .
$$

Proof. Suppose $t<1$. Let

$$
u=\left(1+\epsilon^{2}\right)^{-1 / 2}, \quad v=u^{k}+t\left(1-u^{k}\right),
$$

note that $u<1$ and $v<1$, and choose $\delta>0$ so that

$$
\mathcal{L}_{k}\left(\left\{z \mid M\left(p_{n}^{k} \circ R \circ f, \Delta_{f}(A, r), z\right) \geqq i\right\}\right) \geqq v \alpha(k) r^{k}
$$

whenever $0<r<\delta$.

Suppose $0<r<\delta$. For $j=1,2,3, \cdots$ we define

$$
\begin{aligned}
& Z_{j}=E_{k} \cap\left\{z\left|r u^{i}<\right| z-\left(p_{n}^{k} \circ R \circ l_{f}\right)(A) \mid<r u^{j-1}\right\}, \\
& U_{j}=\left\{x \mid\left(p_{n}^{k} \circ R \circ f\right)(x) \in Z_{j}\right\},
\end{aligned}
$$


and easily verify that $U_{j} \cap \Delta_{f}\left(A, r u^{i-1}\right) \subset U_{j} \cap T(r)$. Hence $z \in Z_{j}$ implies

$$
\begin{aligned}
M\left(p_{n}^{k} \circ R \circ f, \Delta_{f}\left(A, r u^{j-1}\right), z\right) & =M\left(p_{n}^{k} \circ R \circ f, U_{j} \cap \Delta_{f}\left(A, r u^{j-1}\right), z\right) \\
& \leqq M\left(p_{n}^{k} \circ R \circ f, U_{j} \cap T(r), z\right) \\
& =M\left(p_{n}^{k} \circ R \circ f, T(r), z\right),
\end{aligned}
$$

and we infer that

$$
\begin{aligned}
& Z_{j} \cap\left\{z \mid M\left(p_{n}^{k} \circ R \circ f, T(r), z\right) \geqq i\right\} \\
& \supset\left\{z \mid M\left(p_{n}^{k} \circ R \circ f, \Delta_{f}\left(A, r u^{j-1}\right), z\right) \geqq i\right\}-K\left[\left(p_{n}^{k} \circ R \circ l_{f}\right)(A), r u^{i}\right], \\
& \mathcal{L}_{k}\left(Z_{j} \cap\left\{z \mid M\left(p_{n} \circ R \circ f, T(r), z\right) \geqq i\right\}\right) \\
& \geqq v \alpha(k)\left(r u^{j-1}\right)^{k}-\alpha(k)\left(r u^{i}\right)^{k}=\left(u^{k}\right)^{i-1}\left(v-u^{k}\right) \alpha(k) r^{k} .
\end{aligned}
$$

Since the sets $Z_{j}$ are disjoint and $\mathcal{L}_{k}$ measurable, summation with respect to $j$ yields the inequality

$$
\mathcal{L}_{k}\left(\left\{z \mid M\left(p_{n}^{k} \circ R \circ f, T(r), z\right) \geqq i\right\}\right) \geqq \frac{\left(v-u^{k}\right) \alpha(k) r^{k}}{1-u^{k}}=t \alpha(k) r^{k} .
$$

\section{Theorem 6.9. Suppose}

$$
\begin{gathered}
f \in C_{n}(X), V \text { is an open subset of } \mathcal{X}_{f}, \\
\lambda_{k}\left(f,\left\{x \mid m_{f}(x) \in V\right\}\right)=\int_{V} L_{k}^{*}(f, A) d H_{f}^{k} A<\infty, \\
P=V \cap\left\{A \mid L_{k}^{*}(f, A)>0\right\}, \quad H_{f}^{k}(P)<\infty, \\
Q=V \cap\left\{A \mid \varepsilon_{k}(f, A)>0\right\} .
\end{gathered}
$$

Then:

$P$ and $Q$ are analytic subsets of $\mathcal{X}_{f}$, and $Q \subset P$.

(2) $\quad \lim _{r \rightarrow 0+} \frac{H_{f}^{k}\left(Q \cap m_{f}\left[\Delta_{f}(A, r)\right]\right)}{\alpha(k) r^{k}}=1 \quad$ for $H_{f}^{k}$ almost all $A$ in $Q$.

(3) $\quad H_{f}^{k}(W)=\int_{E_{n}} N\left(l_{f}, W, y\right) d \mathcal{F C}_{n}^{k} y \quad$ for every analytic set $W \subset Q$.

$$
\int_{Q} g(A) d H_{f}^{k} A=\int_{E_{n}} \sum_{A \in Q, g(A)=y} g(A) d \mathcal{F}_{n}^{k} y
$$

for every analytically measurable function $g$ on $\mathcal{X}_{f}$. 


$$
L_{k}^{*}(f, A)=L_{* k}(f, A) \text { for } H_{f}^{k} \text { almost all } A \text { in } Q .
$$

(6) For $H_{f}^{k}$ almost all $A$ in $Q$ the following implication holds:

If $i>0, R \in G_{n}$, and $p_{n}^{k} \circ R$ is an $i$-fold essential projection for $f$ at $A$ in dimension $k$, then $\square_{n}^{k}\left[R, l_{f}(A)\right]$ is a Hausdorff $k$ tangent plane of $l_{f}(Q)$ at $l_{f}(A)$.

(7) $l_{f}(Q)$ is a Hausdorff $k$ rectifiable subset of $E_{n}$.

Proof of (2). Since $H_{f}^{k}(Q)<\infty$ and $H_{f}^{k}(P-Q)<\infty$, we have

$$
\limsup _{r \rightarrow 0+} \frac{H_{f}^{k}\left(Q \cap m_{f}\left[\Delta_{f}(A, r)\right]\right)}{\alpha(k) r^{k}} \leqq 1
$$

and

$$
\lim _{r \rightarrow 0+} \frac{H_{f}^{k}\left(P \cap m_{f}\left[\Delta_{f}(A, r)\right]-Q\right)}{\alpha(k) r^{k}}=0
$$

for $H_{f}^{\boldsymbol{k}}$ almost all $A$ in $Q$.

On the other hand if $A$ is any point of $Q$, then there exist $i>0$ and $R \in G_{n}$ such that $p_{n}^{k} \circ R$ is an $i$-fold essential projection for $f$ at $A$ in dimension $k$. For any positive number $r$ which is so small that

$$
m_{f}\left[\Delta_{f}(A, r)\right] \subset V
$$

we infer with the help of the theorems 6.3 and 6.5 that

$$
\begin{aligned}
& \mathcal{L}_{k}\left(\left\{z \mid M\left(p_{n}^{k} \circ R \circ f, \Delta_{f}(A, r), z\right)\right.\right.\geqq i\} \\
&\left.-\left(p_{n}^{k} \circ R \circ l_{f}\right)\left(P \cap m_{f}\left[\Delta_{f}(A, r)\right]\right)\right)=0, \\
& H_{f}^{k}\left(P \cap m_{f}\left[\Delta_{f}(A, r)\right]\right) \geqq \mathcal{L}_{k}\left(p_{n}^{k} \circ R \circ l_{f}\right)\left(P \cap m_{f}\left[\Delta_{f}(A, r)\right]\right) \\
& \geqq \mathcal{L}_{k}\left(\left\{z \mid M\left(p_{n}^{k} \circ R \circ f, \Delta_{f}(A, r), z\right) \geqq i\right\}\right) .
\end{aligned}
$$

It follows that

$$
\liminf _{r \rightarrow 0+} \frac{H_{f}^{k}\left(P \cap m_{f}\left[\Delta_{f}(A, r)\right]\right)}{\alpha(k) r^{k}} \geqq 1 .
$$

Proof of (3). Inasmuch as we already know that

$$
\int_{E_{k}} N\left(l_{f}, W, y\right) d \mathcal{F}_{n}^{k} y \leqq H_{f}^{k}(W),
$$

it is sufficient to prove the following statement: 
If $0<t<1$ and $\epsilon>0$, then there is a partition $F$ of $W$ such that

$$
\begin{gathered}
\sup _{Z \in F} \operatorname{diameter} Z<\epsilon, \\
\sum_{T \in F} \mathcal{H C}_{n}^{k}\left[l_{f}(T)\right] \geqq t^{2} H_{f}^{k}(W)-\epsilon .
\end{gathered}
$$

To prove this we choose an open subset $U$ of $\mathcal{X}_{f}$ for which

$$
W \subset U \subset V, \quad H_{f}^{k}(U \cap P-W)<\epsilon
$$

and let $G$ be the family of all sets $Z$ such that

$$
\begin{gathered}
\text { Closure } m_{f}\left[\Delta_{f}(A, r)\right]=Z \subset U, \quad A \in W, \quad 0<2 r<\epsilon, \\
t \alpha(k) r^{k}<\mathcal{F C}_{n}^{k}\left[l_{f}(Z \cap P)\right] \leqq H_{f}^{k}(Z \cap P)<t^{-1} \alpha(k) r^{k}
\end{gathered}
$$

for some $A$ and $r$. Then $G$ covers $H_{f}^{k}$ almost all of the $W$ in the sense of Vitali, and the Vitali covering theorem [M, 4.1] yields a countable disjointed subfamily $H$ of $G$ such that

$$
H_{f}^{k}\left(W-\underset{z \in \in_{H}}{\bigcup} Z\right)=0 .
$$

We let

$$
F_{1}=\{Z \cap W \mid Z \in H\},
$$

choose such a partition $F_{2}$ of $W-\cup_{z \in{ }_{H}} Z$ that

$$
\sup _{T \in F_{2}} \operatorname{diameter} T<\epsilon,
$$

and define

$$
F=F_{1} \cup F_{2} .
$$

It follows that

$$
\begin{aligned}
t^{2} H_{f}^{k}(W) & \leqq \sum_{Z \in H} t^{2} H_{f}^{k}(Z \cap P) \leqq \sum_{Z \in H} \mathcal{F C}_{n}^{k}\left[l_{f}(Z \cap P)\right] \\
& \leqq \sum_{Z \in H}\left(\mathcal{F C}_{n}^{k}\left[l_{f}(Z \cap W)\right]+H_{f}^{k}(Z \cap P-W)\right) \\
& \leqq \sum_{T \in F} \mathcal{F C}_{n}^{k}\left[l_{f}(T)\right]+H_{f}^{k}(U \cap P-W) \\
& \leqq \sum_{T \in F} \mathcal{F C}_{n}^{k}\left[l_{f}(T)\right]+\epsilon .
\end{aligned}
$$

Proof of (5). By virtue of (2) the usual theorem on the differentia- 
tion of an indefinite integral is valid on $Q$. We infer, for $H_{f}^{k}$ almost all $A$ in $Q$, that

$$
L_{k}^{*}(f, A)=\lim _{r \rightarrow 0+} \frac{\int_{m_{f}\left[\Delta_{f}(A, r)\right]} L_{k}^{*}(f, A) d H_{f}^{k} A}{H_{f}^{k}\left(Q \cap m_{f}\left[\Delta_{f}(A, r)\right]\right)}
$$

and use Theorem 6.5 and (2) to conclude that the limit on the right is equal to

$$
\lim _{r \rightarrow 0+} \frac{\lambda_{k}\left[f, \Delta_{f}(A, r)\right]}{\alpha(k) r^{k}} .
$$

Proof of (6). Defining

$$
\begin{aligned}
Y & =\left\{y \mid 2^{-k} \leqq{ }_{n}^{k}\left[l_{f}(Q), y\right] \leqq \odot_{n}^{k}\left[l_{f}(P), y\right] \leqq 1\right\}, \\
W & =Q \cap\left\{A \mid l_{f}(A) \in Y\right\},
\end{aligned}
$$

we find that

$$
\begin{gathered}
\mathcal{F C}_{n}^{k}\left[l_{f}(Q)\right] \leqq \mathcal{F C}_{n}^{k}\left[l_{f}(P)\right]<\infty, \quad \mathcal{F C}_{n}^{k}\left[l_{f}(Q)-Y\right]=0, \\
H_{f}^{k}[Q-W]=\int_{E_{n}} N\left(l_{f}, Q-W, y\right) d \mathcal{K}_{n}^{k} y=0 .
\end{gathered}
$$

Now suppose $A \in W, i$ is a positive integer, and the hypotheses of Theorem 6.8 hold. Applying the Theorems 6.3 and 6.5 as in the proof of (2) we conclude, for all sufficiently small positive numbers $r$, that

$$
\begin{aligned}
\mathcal{L}_{k}\left(\left\{z \mid M\left(p_{n}^{k} \circ R \circ f,\right.\right.\right. & T(r), z) \geqq i\}) \\
& \leqq \mathcal{L}_{k}\left[\left(p_{n}^{k} \circ R \circ l_{f}\right)\left(P \cap m_{f}[T(r)]\right)\right] \\
& \leqq \mathcal{F C}_{n}^{k}\left[l_{f}\left(P \cap m_{f}[T(r)]\right)\right] \leqq \mathcal{F C}_{n}^{k}\left(l_{f}(P) \cap f[T(r)]\right) \\
& \leqq \mathcal{F C}_{n}^{k}\left(l_{f}(P) \cap \diamond_{n}^{k}\left[R, \epsilon, l_{f}(A)\right] \cap K\left[l_{f}(A), r\right]\right) .
\end{aligned}
$$

It follows that

$$
\begin{aligned}
& \odot_{n}^{k}\left[l_{f}(P) \cap \diamond_{n}^{k}\left[R, \epsilon, l_{f}(A)\right], l_{f}(A)\right] \geqq 1, \\
& \odot_{n}^{k}\left[l_{f}(P)-\diamond_{n}^{k}\left[R, \epsilon, l_{f}(A)\right], l_{f}(A)\right]=0 .
\end{aligned}
$$

Theorem 6.10. Suppose $f \in C_{k}(X)$ and $U$ is the union of all those open subsets $V$ of $\mathcal{X}_{f}$ for which

$$
\lambda_{k}\left(f,\left\{x \mid m_{f}(x) \in V\right\}\right)<\infty .
$$


Then:

$$
\begin{aligned}
& L_{k}^{*}(f, A) \geqq \limsup _{r \rightarrow 0+} D\left[f, r, \Delta_{f}(A, r)\right] \text { for } A \in M_{f .} \\
& \mathcal{E}_{k}(f, A) \geqq \liminf _{r \rightarrow 0+} D\left[f, r, \Delta_{f}(A, r)\right] \text { for } A \in M_{f} .
\end{aligned}
$$

(3) If $W$ is an open subset of $\mathcal{H}_{f}, y \in E_{k}, r>0$, and $Z$ is an essential canonical region of $\left(f,\left\{x \mid m_{f}(x) \in W\right\}, y, r\right)$, then there is an $A \in W$ for which $l_{f}(A)=y, \Delta_{f}(A, r)=Z$, and

$$
\limsup _{t \rightarrow 0+} D\left[f, t, \Delta_{f}(A, t)\right] \geqq 1 \text {. }
$$

(4) If $W$ is an open subset of $\mathcal{X}_{f}, A \in W$, and

$$
\underset{r \rightarrow 0+}{\lim \inf } D\left[f, r, \Delta_{f}(A, r)\right]<\underset{r \rightarrow 0+}{\lim \sup } D\left[f, r, \Delta_{f}(A, r)\right],
$$

then $M\left(f,\left\{x \mid m_{f}(x) \in W\right\}, l_{f}(A)\right)=\infty$.

(5) If $W$ is an open subset of $\mathscr{X}_{f}$ and $y \in E_{k}$, then

$$
\sum_{A \in W, l_{f}(A)=y} L_{k}^{*}(f, A) \geqq M\left(f,\left\{x \mid m_{f}(x) \in W\right\}, y\right) .
$$

(6) If $W$ is an open subset of $\mathcal{X}_{f}, y \in E_{k}$, and

$$
M\left(f,\left\{x \mid m_{f}(x) \in W\right\}, y\right)<\infty,
$$

then

$$
M\left(f,\left\{x \mid m_{f}(x) \in W\right\}, y\right)=\sum_{A \in W, l_{f}(A)=y} \lim _{r \rightarrow 0+} D\left[f, r, \Delta_{f}(A, r)\right] .
$$

(7) If $W$ is an open subset of $\mathcal{X}_{f}$, then

$$
\begin{aligned}
& \lambda_{k}\left(f,\left\{x \mid m_{f}(x) \in W\right\}\right)=\int_{W} L_{k}^{*}(f, A) d H_{f}^{k} A \\
& =\int_{E_{k}} \sum_{A \in W, l_{f}(A)=y} L_{k}^{*}(f, y) d \mathcal{L}_{k} y \\
& =\int_{E_{k}} M\left(f,\left\{x \mid m_{f}(x) \in W\right\}, y\right) d \mathcal{L}_{k} y \text {. }
\end{aligned}
$$

(8) For $\mathcal{L}_{k}$ almost all $y$ in $E_{k}$ the following implication holds: If $W$ is an open subset of $U$, then

$$
M\left(f,\left\{x \mid m_{f}(x) \in W\right\}, y\right)=\sum_{A \in W, l_{f}(A)=\nu} L_{k}^{*}(f, A) .
$$




$$
L_{k}^{*}(f, A)=L_{* k}(f, A)=\varepsilon_{k}(f, A)=\lim _{r \rightarrow 0+} D\left[f, r, \Delta_{f}(A, r)\right]<\infty
$$

for $H_{f}^{k}$ almost all $A$ in $U$.

$$
L_{k}^{*}(f, A)=L_{* k}(f, A)=\infty \text { for } A \in \mathcal{X}_{f}-U .
$$

PRoOF of (1) AND (2). If $A \in \mathscr{X}_{f}, r>0$, and

$$
D\left[f, r, \Delta_{f}(A, r)\right] \geqq i,
$$

then

$$
\begin{aligned}
M\left[f, \Delta_{f}(A, r), y\right] & \geqq i \text { for } y \in K\left[l_{f}(A), r\right], \\
\lambda_{k}\left[f, \Delta_{f}(A, r)\right] & \geqq i \mathcal{L}_{k}\left(\left\{y \mid M\left[f, \Delta_{f}(A, r), y\right] \geqq i\right\}\right)=i \alpha(k) r^{k} .
\end{aligned}
$$

Proof of (3). We define $Z_{0}, Z_{1}, Z_{2}, Z_{3}, \cdots$ inductively in such a way that $Z_{0}=Z$ and $Z_{i}$ is an essential canonical region of $\left(f,\left\{x \mid m_{f}(x) \in W\right\}, y, r 2^{-i}\right)$ with $Z_{i} \subset Z_{i-1}$ for every positive integer $i$. We infer that Closure $Z_{i}$ is a compact subset of $Z_{i-1}$ for every positive integer $i$, and choose

$$
a \in \bigcap_{i=0}^{\infty} \operatorname{Closure} Z_{i}=\bigcap_{i=0}^{\infty} Z_{i}, \quad A=m_{f}(a) .
$$

It follows that

$$
\begin{gathered}
a \in Z \subset\left\{x \mid m_{f}(x) \in W\right\}, \quad A \in W, \\
l_{f}(A)=f(a)=y, \quad \Delta_{f}(A, r)=\Delta_{f}(a, r)=Z, \\
\Delta_{f}\left(A, r 2^{-i}\right)=Z_{i} \text { for } i=1,2,3, \cdots .
\end{gathered}
$$

Proof of (4). There exist positive numbers

$$
r_{0}>r_{1}>r_{2}>r_{3}>\cdots
$$

such that

$$
D\left[f, r_{i-1}, \Delta_{f}\left(A, r_{i-1}\right)\right] \neq D\left[f, r_{i}, \Delta_{f}\left(A, r_{i}\right)\right]
$$

for $i=1,2,3, \cdots$. Hence we can associate with each positive integer $i$ an essential canonical region $Z_{i}$ of $\left(f,\left\{x \mid m_{f}(x) \in W\right\}, l_{f}(A), r_{i}\right)$ such that

$$
Z_{i} \subset \Delta_{f}\left(A, r_{i-1}\right)-\Delta_{f}\left(A, r_{i}\right) .
$$

Since $Z_{1}, Z_{2}, Z_{3}, \cdots$ are disjoint subsets of $\left\{x \mid m_{f}(x) \in W\right\}$ and

$$
S\left[f, Z_{i}, l_{f}(A)\right] \geqq 1 \quad \text { for } i=1,2,3, \cdots,
$$

we conclude that 
$M\left[f,\left\{x \mid m_{f}(x) \in W\right\}, l_{f}(A)\right] \geqq S\left[f,\left\{x \mid m_{f}(x) \in W\right\}, l_{f}(A)\right]=\infty$.

ProOf OF (5) AND (6). By virtue of (3) we have

$$
M\left(f,\left\{x \mid m_{f}(x) \in W\right\}, y\right)=\lim _{r \rightarrow 0+} \sum_{A \in W, l_{f}(A)=y} D\left[f, r, \Delta_{f}(A, r)\right]
$$

whenever $W$ is an open subset of $\mathcal{X}_{f}$ and $y \in E_{k}$.

Hence (1) implies (5), and (4) implies (6).

PROOF OF (7). Consider the statement obtained from (7) upon replacing "=" by " $\geqq$ " in front of each of the three integral signs. This is a true statement by virtue of Theorem 6.2 , the remark following that theorem, and Proposition (5) of the present theorem. However the first and last term in this string of inequalities are equal by Lemma 6.4.

Proof of (8). Let $B$ be such a countable basis for the topology of $U$ that

$$
\lambda_{k}\left(f,\left\{x \mid m_{f}(x) \in V\right\}\right)<\infty \text { for } V \in B
$$

and let

$$
Z_{V}=\left\{y \mid M\left(f,\left\{x \mid m_{f}(x) \in V\right\}, y\right)<\sum_{A \in V, l_{f}(A)=y} L_{k}^{*}(f, A)\right\}
$$

for $V \in B$. From (5) and (7) we infer that

$$
\begin{gathered}
\mathcal{L}_{k}\left(Z_{V}\right)=0 \text { for } V \in B, \\
\mathcal{L}_{k}\left(\bigcup_{V \in B} Z_{V}\right)=0 .
\end{gathered}
$$

Now suppose

$$
y \in E_{k}-\bigcup_{V \in B} Z_{V}
$$

and $W$ is any open subset of $\mathscr{X}_{f}$.

If $A_{1}, A_{2}, \cdots, A_{m}$ are distinct elements of $W$ such that $l_{f}\left(A_{i}\right)=y$ for $i=1,2, \cdots, m$, then there exist disjoint elements $V_{1}, V_{2}, \cdots$, $V_{m}$ of $B$ such that $A_{i} \in V_{i}$ for $i=1,2, \cdots, m$. It follows that

$$
\begin{aligned}
\sum_{i=1}^{m} L_{k}^{*}\left(f, A_{i}\right) & \leqq \sum_{i=1}^{m} \sum_{A} \sum_{V_{i}, l_{f}(A)=y} L_{k}^{*}(f, A) \\
& =\sum_{i=1}^{m} M\left(f,\left\{x \mid m_{f}(x) \in V_{i}\right\}, y\right) \\
& \leqq M\left(f,\left\{x \mid m_{f}(x) \in W\right\}, y\right) .
\end{aligned}
$$


Proof of (9). We see from (1), (2), and Theorem 6.7 that

$$
\begin{aligned}
& \liminf _{r \rightarrow 0+} D\left[f, r, \Delta_{f}(A, r)\right] \leqq \limsup _{r \rightarrow 0+} D\left[f, r, \Delta_{f}(A, r)\right] \leqq L_{k}^{*}(f, A), \\
& \liminf _{r \rightarrow 0+} D\left[f, r, \Delta_{f}(A, r)\right] \leqq \mathcal{E}_{k}(f, A) \leqq L_{* k}(f, A) \leqq L_{k}^{*}(f, A)
\end{aligned}
$$

whenever $A \in \mathcal{X}_{f}$. Choosing $B$ as in the proof of (8) we shall complete the proof by showing that

$$
\int_{V} \liminf _{r \rightarrow 0+} D\left[f, r, \Delta_{f}(A, r)\right] d H_{f}^{k} A \geqq \int_{V} L_{k}^{*}(f, A) d H_{f}^{k} A
$$

whenever $V \in B$.

Suppose $V \in B$. By (6) and (7) we conclude that

$$
\begin{aligned}
& \int_{V} \liminf _{r \rightarrow 0+} D {\left[f, r, \Delta_{f}(A, r)\right] d H_{f}^{k} A } \\
& \geqq \int_{E_{k}} \sum_{A} \in \sum_{V, l_{f}(A)=\nu} \liminf _{r \rightarrow 0+} D\left[f, r, \Delta_{f}(A, r)\right] d \mathcal{L}_{k} y \\
& \quad=\int_{E_{k}} M\left(f,\left\{x \mid m_{f}(x) \in V\right\}, y\right) d \mathcal{L}_{k} y=\int_{V} L_{k}^{*}(f, A) d H_{f}^{k} A .
\end{aligned}
$$

7. Fréchet equivalence. If $f \in C_{n}(X)$ and $T$ is a homeomorphism of $X$ onto $X$, then $f$ and $f \circ T$ behave identically with respect to the geometric properties discussed in the two preceding sections. These mappings have the same multiplicities, the same areas, and isometric middle spaces. However $f \circ T$ may be preferable to $f$ on account of differentiability properties which are not invariant under homeomorphisms of $X$.

This passage from a mapping to a geometrically equivalent one, which may have superior analytic properties, can be extended by a limiting process. If $f \in C_{n}(X)$ and $T_{1}, T_{2}, T_{3}, \cdots$ are homeomorphisms of $X$ onto $X$ such that $f \circ T_{i}$ converges as $i$ tends to infinity, then the limit mapping is geometrically very similar to $f$ but may be smoother and easier to compute with than $f$. An example is the familiar process of reparametrization by arc length which replaces an arbitrary curve of finite length by one satisfying a Lipschitz condition.

The formal definition follows:

Suppose $f \in C_{n}(X)$ and $g \in C_{n}(X)$. We say that

$$
f \text { and } g \text { are Fréchet equivalent }
$$


if and only if there exist homeomorphisms $T_{1}, T_{2}, T_{3}, \cdots$ of $X$ onto $X$ such that for each compact subset $Q$ of $X$

$\left(f \circ T_{i}\right)(x) \rightarrow g(x)$ uniformly for $x \in Q$ as $i \rightarrow \infty$, $\left(g \circ T_{i}^{-1}\right)(x) \rightarrow f(x)$ uniformly for $x \in Q$ as $i \rightarrow \infty$,

Closure $\bigcup_{i=1}^{\infty}\left[T_{i}(Q) \cup T_{\S}^{-1}(Q)\right]$ is compact.

It is easy to verify that we have indeed defined an equivalence relation on $C_{n}(X)$. However, most important for the theory of area is the following proposition:

If $f$ and $g$ are Fréchet equivalent elements of $C_{n}(X)$, then there exist homeomorphisms $T_{1}, T_{2}, T_{3}, \cdots$ of $X$ onto $X$ and an isometry $u$ of $\mathfrak{X}_{g}$ onto $\mathfrak{X X}_{f}$ such that

$$
l_{f} \circ u=l_{g}
$$

and such that for each compact subset $Q$ of $X$

$$
\begin{gathered}
\left(m_{f} \circ T_{i}\right)(x) \rightarrow\left(u \circ m_{g}\right)(x) \text { uniformly for } x \in Q \text { as } i \rightarrow \infty, \\
\left(m_{g} \circ T_{i}^{-1}\right)(x) \rightarrow\left(u^{-1} \circ m_{f}\right)(x) \text { uniformly for } x \in Q \text { as } i \rightarrow \infty .
\end{gathered}
$$

To prove this we start with a sequence of homeomorphisms $T_{1}, T_{2}, T_{3}, \cdots$ which have the properties stated in the definition of Fréchet equivalence. Later we shall replace this sequence by a subsequence.

If $a \in X, a^{\prime} \in X^{\prime}$, and $\epsilon>0$, then there exist a positive integer $j$ and neighborhoods $V$ and $V^{\prime}$ of $a$ and $a^{\prime}$ such that

$$
d_{f}\left[\left(m_{f} \circ T_{i}\right)(x),\left(m_{f} \circ T_{i}\right)\left(x^{\prime}\right)\right] \leqq d_{g}\left[m_{g}(x), m_{g}\left(x^{\prime}\right)\right]+\epsilon
$$

whenever $x \in V, x^{\prime} \in V^{\prime}, i$ is an integer, and $i>j$.

To check this we choose a continuum $W \subset X$ for which

$$
a \in W, a^{\prime} \in W \text {, diameter } g(W)<d_{g}\left[m_{g}(a), m_{g}\left(a^{\prime}\right)\right]+\epsilon / 5
$$

and secure a positive integer $j$ and open connected subsets $V$ and $V^{\prime}$ of $X$ such that

$a \in V, a^{\prime} \in V^{\prime}$, Closure $V$ and Closure $V^{\prime}$ are compact, $d_{g}\left[m_{g}(x), m_{g}(a)\right]<\epsilon / 5$ and $\left|\left(f \circ T_{i}\right)(x)-g(a)\right|<\epsilon / 5$ for $x \in V$ and $i>j$, $d_{g}\left[m_{g}\left(x^{\prime}\right), m_{g}\left(a^{\prime}\right)\right]<\epsilon / 5$ and $\left|\left(f \circ T_{i}\right)\left(x^{\prime}\right)-g\left(a^{\prime}\right)\right|<\epsilon / 5$ for $x^{\prime} \in V^{\prime}$ and $i>j$, $\left|\left(f \circ T_{i}\right)(x)-g(x)\right|<\epsilon / 5$ for $x \in W$ and $i>j$.

Letting

$$
Z=W \cup \text { Closure } V \cup \text { Closure } V^{\prime}
$$


we conclude for $x \in V, x^{\prime} \in V^{\prime}$ and $i>j$ that

$d_{f}\left[\left(m_{f} \circ T_{i}\right)(x),\left(m_{f} \circ T_{i}\right)\left(x^{\prime}\right)\right]$

$$
\begin{aligned}
& \leqq \text { diameter }\left(f \circ T_{i}\right)(Z) \leqq \text { diameter } g(Z)+2 \epsilon / 5 \\
& \leqq d_{\theta}\left[m_{g}(a), m_{g}\left(a^{\prime}\right)\right]+3 \epsilon / 5 \leqq d_{g}\left[m_{\theta}(x), m_{\theta}\left(x^{\prime}\right)\right]+\epsilon .
\end{aligned}
$$

The preceding equicontinuity property implies the following:

If $Q$ is a compact subset of $X$ and $\epsilon>0$, then there exists a positive integer $j$ such that

$$
d_{f}\left[\left(m_{f} \circ T_{i}\right)(x),\left(m_{f} \circ T_{i}\right)\left(x^{\prime}\right)\right]<d_{g}\left[m_{g}(x), m_{g}\left(x^{\prime}\right)\right]+\epsilon
$$

whenever $x \in Q, x^{\prime} \in Q, i$ is an integer, and $i>j$.

In view of this and also of the fact that

Closure $\left\{\left(m_{f} \circ T_{i}\right)(x) \mid i=1,2,3, \cdots\right\}$ is compact for $x \in X$ we may just as well assume, after passage to a subsequence, that there is a mapping $U$ of $X$ into $M_{f}$ such that

$$
\left(m_{f} \circ T_{i}\right)(x) \rightarrow U(x) \text { uniformly for } x \in Q \text { as } i \rightarrow \infty
$$

whenever $Q$ is a compact subset of $X$.

It follows that

$$
d_{f}\left[U(x), U\left(x^{\prime}\right)\right] \leqq d_{\theta}\left[m_{\theta}(x), m_{\theta}\left(x^{\prime}\right)\right] \text { for } x \in X, x^{\prime} \in X
$$

and that there is a function $u$ on $\mathscr{X}_{\theta}$ to $\mathscr{X}_{f}$ such that

$$
\begin{gathered}
U=u \circ m_{g}, \\
d_{f}\left[u(A), u\left(A^{\prime}\right)\right] \leqq d_{\theta}\left(A, A^{\prime}\right) \text { for } A \in \mathcal{X}_{g}, A^{\prime} \in \mathcal{X}_{g} .
\end{gathered}
$$

Furthermore

$$
\begin{aligned}
\left(l_{f} \circ u\right)\left[m_{g}(x)\right] & =l_{f}\left[\left(u \circ m_{g}\right)(x)\right]=l_{f}\left[\lim _{i \rightarrow \infty}\left(m_{f} \circ T_{i}\right)(x)\right] \\
& =\lim _{i \rightarrow \infty}\left(f \circ T_{i}\right)(x)=g(x)=l_{g}\left[m_{g}(x)\right]
\end{aligned}
$$

for $x \in X$, whence $l_{f} \circ u=l_{g}$.

By symmetry we may also assume, after passage to another subsequence, that there is a function $v$ on $\mathscr{X}_{f}$ to $\mathscr{X}_{\theta}$ such that

$$
\left(m_{\theta} \circ T_{i}^{-1}\right)(x) \rightarrow\left(v \circ m_{f}\right)(x) \text { uniformly for } x \in Q \text { as } i \rightarrow \infty
$$

whenever $Q$ is a compact subset of $X$ and such that

$$
d_{\theta}\left[v(A), v\left(A^{\prime}\right)\right] \leqq d_{f}\left(A, A^{\prime}\right) \text { for } A \in \mathcal{X}_{f}, A^{\prime} \in \mathcal{X}_{f} .
$$

We shall complete the proof by showing that the functions $u$ and $v$ 
are inverse to each other.

Suppose $A \in \mathscr{X}_{f}$ and $\epsilon>0$. Letting

$$
a \in A, b \in v(A), Q=\left\{T_{i}^{-1}(a) \mid i=1,2,3, \cdots\right\} \cup\{b\},
$$

we choose a positive integer $j$ such that

$$
d_{f}\left[\left(m_{f} \circ T_{i}\right)(x),\left(m_{f} \circ T_{i}\right)\left(x^{\prime}\right)\right] \leqq d_{\theta}\left[m_{\theta}(x), m_{\theta}\left(x^{\prime}\right)\right]+\epsilon
$$

whenever $x \in Q, x^{\prime} \in Q$, and $i>j$. We infer that

$$
\begin{aligned}
d_{f}[A,(u \circ v)(A)] & =d_{f}\left[m_{f}(a),\left(u \circ m_{g}\right)(b)\right] \\
& =d_{f}\left[m_{f}(a), \lim _{i \rightarrow \infty}\left(m_{f} \circ T_{i}\right)(b)\right] \\
& =\lim _{i \rightarrow \infty} d_{f}\left[m_{f}(a),\left(m_{f} \circ T_{i}\right)(b)\right] \\
& =\lim _{i \rightarrow \infty} d_{f}\left[\left(m_{f} \circ T_{i}\right)\left[T_{i}^{-1}(a)\right],\left(m_{f} \circ T_{i}\right)(b)\right] \\
& \leqq \lim _{i \rightarrow \infty} \sup d_{g}\left[\left(m_{g} \circ T_{i}^{-1}\right)(a), m_{g}(b)\right]+\epsilon \\
& =d_{g}\left[\left(v \circ m_{f}\right)(a), v(A)\right]+\epsilon=d_{g}[v(A), v(A)]+\epsilon=\epsilon .
\end{aligned}
$$

It follows that

$$
(u \circ v)(A)=A \text { for } A \in \mathscr{X r}_{f} .
$$

Similarly we find that

$$
(v \circ u)(A)=A \text { for } A \in \mathscr{X}_{g} .
$$

The proof is complete.

The preceding proposition has the following corollaries:

If $V$ is an open subset of $\mathcal{X}_{g}$ and $W$ is a compact subset of $\left\{x \mid m_{g}(x) \in V\right\}$, then there is a positive integer $j$ such that

$$
T_{i}(W) \subset\left\{x \mid m_{f}(x) \in u(V)\right\} \text { for } i>j .
$$

If $V$ is an open subset of $\mathcal{X}_{\theta}$, then

$$
\lambda_{k}\left(g,\left\{x \mid m_{g}(x) \in V\right\}\right)=\lambda_{k}\left(f,\left\{x \mid m_{f}(x) \in u(V)\right\}\right) .
$$

If $V$ is an open subset of $\mathscr{X}_{g}, R \in G_{n}$, and $z \in E_{b}$, then

$$
\begin{aligned}
S\left(p_{n}^{k} \circ R \circ g,\left\{x \mid m_{g}(x)\right.\right. & \in V\}, z) \\
& =S\left(p_{n}^{k} \circ R \circ f,\left\{x \mid m_{f}(x) \in u(V)\right\}, z\right), \\
M\left(p_{n}^{k} \circ R \circ g,\left\{x \mid m_{g}(x)\right.\right. & \in V\}, z) \\
& =M\left(p_{n}^{k} \circ R \circ f,\left\{x \mid m_{f}(x) \in u(V)\right\}, z\right) .
\end{aligned}
$$


If $A \in \mathcal{X}_{g}$ and $r>0$, then

$$
u\left(m_{g}\left[\Delta_{g}(A, r)\right]\right)=m_{f}\left(\Delta_{f}[u(A), r]\right) .
$$

If $A \in \mathcal{M}_{g}$, then

$$
\begin{gathered}
L_{k}^{*}(g, A)=L_{k}^{*}[f, u(A)], \quad L_{* k}(g, A)=L_{* k}\lfloor f, u(A)\rfloor, \\
\varepsilon_{k}(g, A)=\varepsilon_{k}[f, u(A)] .
\end{gathered}
$$

If $V \subset \mathcal{X}_{g}$, then $H_{o}^{k}(V)=H_{f}^{k}[u(V)]$.

If $V \subset \mathcal{X}_{o}$ and $y \in E_{n}$, then

$$
N\left(l_{\theta}, V, y\right)=N\left(l_{f}, u(V), y\right) .
$$

It follows that the Hausdorff $N$ areas of $l_{f}$ and $l_{g}$ are equal. However the Hausdorff $N$ areas of $f$ and $g$ need not be equal, and the integralgeometric $N$ areas of $f$ and $g$ need not be equal. The reason why $N(f, X, y)$ may differ from $N(g, X, y)$ is that $N(h, X, y)$ is not lower semi-continuous with respect to $h$ on $C_{n}(X)$. The invariants $S, M$, and $\lambda_{k}$ of Fréchet equivalence are lower semi-continuous functions of compact origin.

We observe also that if $\dot{X}$ is the set of all those points of $X$ which do not have a $k$-cell neighborhood in $X$ and if

$$
\mathscr{X}_{f}=\mathscr{X}_{f} \cap\left\{A \mid \Delta_{f}(A, r) \cap \dot{X} \neq 0 \text { for all } r>0\right\},
$$

then $u\left(\mathscr{X}_{g}\right)=\mathscr{X}_{f}$.

A cyclic mapping is a function $f \in C_{n}(X)$ whose middle space $\mathcal{X C}_{f}$ has no cut point. A nondegenerate mapping is a cyclic mapping $f$ such that $\mathcal{H}_{f}^{\circ}$ has more than one element.

For example a cyclic mapping of a two-cell $X \subset E_{2}$ is one whose continua of constancy do not separate $X$, while a nondegenerate mapping is one whose continua of constancy separate neither $X$ nor $E_{2}$.

The usefulness of the concept of Fréchet equivalence for the theory of two-dimensional Lebesgue area is largely due to the following proposition of C. B. Morrey ([MCB], [CE 4]):

If $X$ is a two-cell, $f \in C_{n}(X), L_{2}(f)<\infty$, and $f$ is a nondegenerate mapping, then $f$ has a Fréchet equivalent $g$ whose partial derivatives $D_{1} g(x)$ and $D_{2} g(x)$ exist for $\mathcal{L}_{2}$ almost all $x$ in $X-\dot{X}$ and whose Lebesgue area $L_{2}(g)$ equals the Dirichlet integral

$$
\frac{1}{2} \int_{X-\dot{x}}\left|D_{1} g(x)\right|^{2}+\left|D_{2} g(x)\right|^{2} d \mathcal{L}_{2} x
$$

This proposition makes the techniques of classical analysis avail- 
able for the discussion of all Fréchet invariant properties of nondegenerate mappings of two-cells with finite Lebesgue area. Furthermore there are two devices by which results concerning certain geometric properties can be extended from nondegenerate to arbitrary mappings of two-cells.

If $X$ is a two-cell, $f \in C_{n}(X)$, and $r_{1}, r_{2}, r_{3}, \cdots$ are the monotone retractions of $\mathcal{X}_{f}$ onto its proper cyclic elements, then

$$
\left(l_{f} \circ r_{1} \circ m_{f}\right),\left(l_{f} \circ r_{2} \circ m_{f}\right),\left(l_{f} \circ r_{3} \circ m_{f}\right), \cdots
$$

are cyclic mappings in $C_{n}(X)$. They are called the cyclic components of $f$. Certain properties possessed by every cyclic component of $f$ are inherited by $f$. Such properties of mappings are said to be cyclically extensible (see [R 5]).

If $X$ is a two-cell, $f \in C_{n}(X)$, and $f$ is a cyclic but not a nondegenerate mapping, then $f$ maps $\dot{X}$ onto a single point $y \in E_{n}$. For each $\epsilon>0$ there exists a two-cell $T$ such that

$$
\{x|| f(x)-y \mid \geqq \epsilon\} \subset T \subset X-\dot{X} .
$$

The mapping $(f \mid T) \in C_{n}(T)$ is called an internal approximation of $f$. Every cyclic component of $(f \mid T)$ is a nondegenerate mapping in $C_{n}(T)$. Certain properties possessed by every internal approximation of $f$ are inherited by $f$. Such properties of mappings are said to be internally extensible.

Now if a property of mappings of two-cells is both cyclically and internally extensible and if it is possessed by every nondegenerate mapping of finite Lebesgue area, then this property is successively inherited by all internal approximations of those cyclic mappings of finite Lebesgue area which fail to be nondegenerate, by all cyclic mappings of finite Lebesgue area, and by all mappings of finite Lebesgue area.

8. Mappings of locally connected finitely connected plane continua. For any two subsets $A$ and $B$ of $E_{2}$ we define

$$
\gamma(A, B)=\sup _{x \in B} \inf _{\nu \in A}|x-y|
$$

and the Hausdorff distance

$$
\rho(A, B)=\sup \{\gamma(A, B), \gamma(B, A)\} .
$$

Letting

$$
\begin{aligned}
& \Gamma=\left\{A \mid A \text { is a nonvacuous compact subset of } E_{2}\right\}, \\
& \Omega=\Gamma \cap\{A \mid A \text { is connected }\},
\end{aligned}
$$


we note that $\Gamma$ is a boundedly compact metric space with respect to the Hausdorff distance, and that the set $\Omega$ of all plane continua is a closed subset of $\Gamma$.

With each $A \subset E_{2}$ we associate

$$
\begin{aligned}
A^{\#} & =\text { the union of all bounded components of } E_{2}-A, \\
\sigma(A) & =\inf \left\{\gamma(A, W) \mid W \text { is a component of } E_{2}-A\right\} .
\end{aligned}
$$

The following theorem characterizes the finitely connected compact subsets of the plane by means of the function $\sigma$.

Theorem 8.1. If $A \in \Gamma$, then

$$
\sigma(A)>0
$$

if and only if the number of components of $E_{2}-A$ is finite.

Proof. Since $\gamma(A, W)>0$ for every component $W$ of $E_{2}-A$, the second condition implies the first.

Now suppose $\sigma(A)>0$. Then each component of $E_{2}-A$ contains an open circular disc of radius $\sigma(A)$, whence

$\pi \cdot[\sigma(A)]^{2} \cdot$ (number of bounded components of $\left.E_{2}-A\right) \leqq \mathcal{L}_{2}\left(A^{\#}\right)<\infty$, because $A^{\#}$ is bounded. Hence the number of bounded components of $E_{2}-A$ is finite. Furthermore $E_{2}-A$ has precisely one unbounded component.

Lemma 8.2. If $A$ and $B$ are such subsets of $E_{2}$ that

$$
\gamma(A, B)<\sigma(A) \text { and Boundary } B \subset A,
$$

then

$$
B \subset A \text {. }
$$

Proof. Otherwise $E_{2}-A$ has a component $W$ which meets $B$. It follows that

$W \cap$ Closure $(W \cap B) \cap$ Closure $(W-B)$

$C W \cap$ Boundary $B \subset W \cap A=0$,

$W-B=0, \quad W \subset B, \quad \sigma(A) \leqq \gamma(A, W) \leqq \gamma(A, B)<\sigma(A)$.

LEMMa 8.3. If

$A \in \Omega, \quad B \in \Omega, \quad A \cap B=0, \quad A^{*} \cap B^{*} \neq 0$,

then

$$
\text { either } A \subset B^{\sharp} \text { or } B \subset A^{\sharp} \text {. }
$$


Proof. If $A \nsubseteq B^{\#}$, then $A \cap B^{\#}=0$ because the connected set $A$, which does not meet $B$, is contained in a single component of $E_{2}-B$. It follows that

$$
A \cap\left(B \cup B^{*}\right)=0 .
$$

If also $B \subset A^{\#}$, then

$$
A^{\#} \cap\left(B \cup B^{\#}\right)=0
$$

because the connected set $B \cup B^{\#}$, which does not meet $A$, is contained in a single component of $E_{2}-A$ but not in $A^{\#}$. It follows that $A^{\#} \cap B^{\#}=0$, contrary to hypothesis.

LEMMA 8.4. If

$$
A \in \Gamma, \quad B \in \Omega, \quad A \subset B^{\#},
$$

then

$$
A \cup A^{\#} \subset B^{\#} .
$$

Proof. The unbounded connected set $E_{2}-B^{\#}$, which does not meet $A$, is contained in the unbounded component of $E_{2}-A$. Thus

$$
E_{2}-B^{\#} \subset E_{2}-\left(A \cup A^{\#}\right), \quad A \cup A^{\#} \subset B^{\sharp} .
$$

Lemma 8.5. If $A \in \Gamma, A^{\sharp}=0$, and $\epsilon>0$, then there is a $\delta>0$ such that

$$
\gamma\left(A, B^{\#}\right) \leqq \epsilon \text { whenever } \gamma(A, B)<\delta .
$$

Proof. We choose $r>0$ so that

$$
A \subset\{x|| x \mid<r\}
$$

and let

$$
f(x)=\inf _{\nu \in A}|x-y| \quad \text { for } x \in E_{2} .
$$

Each point of the compact set

$$
V=\{x|| x \mid \leqq r \text { and } f(x) \geqq \epsilon\}
$$

is in some unbounded connected open set $W$ whose closure does not meet $A$. Hence $V$ is contained in the union of finitely many such sets $W_{1}, W_{2}, \cdots, W_{m}$. Defining

$$
\begin{aligned}
& Z=\{x|| x \mid \geqq r\} \cup \bigcup_{i=1}^{m} W_{i}, \\
& \delta=\inf _{x \in Z} f(x),
\end{aligned}
$$


we see that $\delta>0$ and that $Z$ is an unbounded connected set for which

$$
\{x \mid f(x) \geqq \epsilon\} \subset Z \text {. }
$$

If $\gamma(A, B)<\delta$, then $Z$ does not meet $B$ and is contained in the unbounded component of $E_{2}-B$. Thus

$$
Z \subset E_{2}-B^{\#}, \quad B^{\#} \subset E_{2}-Z \subset\{x \mid f(x)<\epsilon\}, \quad \gamma\left(A, B^{\#}\right) \leqq \epsilon .
$$

Lemma 8.6. If $f$ maps the topological space $X$ into the circle $Y$ and if $X$ has connected subsets $A, B, C$ such that

$$
f(A \cap B) \nsubseteq f(C), \quad f(B \cap C) \nsubseteq f(A), \quad f(C \cap A) \nsubseteq f(B),
$$

then $f$ is an essential map of $X$ into $Y$.

Proof. Otherwise $f=h \circ g$ where $g$ is a map of $X$ into $E_{1}$ and $h$ is a covering map of $E_{1}$ onto $Y$.

Choosing $a, b, c$ so that

$$
\begin{array}{ll}
a \in B \cap C, & f(a) \oplus f(A), \\
b \in C \cap A, & f(b) \notin f(B), \\
c \in A \cap B, & f(c) \notin f(C),
\end{array}
$$

we note that $g(a), g(b), g(c)$ are distinct and that one of these three real numbers lies between the two others.

If $g(a)$ is between the numbers $g(b)$ and $g(c)$, both of which are in the interval $g(A)$, then $g(a) \in g(A)$ and $f(a) \in f(A)$, contrary to our choice of $a$.

The other two alternatives can be similarly eliminated.

Lemma 8.7. If $g$ maps the topological space $X$ into

$$
Z=E_{2}-\{(\xi, \eta)\}
$$

and if $X$ has connected subsets $E, W, N, S$ such that

$$
\begin{aligned}
& E \cap N, N \cap W, W \cap S, S \cap E \text { are nonvacuous, } \\
& g(E) \subset\{(u, v) \mid u>\xi\}, \quad g(W) \subset\{(u, v) \mid u<\xi\}, \\
& g(N) \subset\{(u, v) \mid v>\eta\}, \quad g(S) \subset\{(u, v) \mid v<\eta\},
\end{aligned}
$$

then $g$ is an essential map of $X$ into $Z$.

PRoof. The preceding lemma is applicable with

$$
\begin{aligned}
& f(x)=\frac{g(x)-(\xi, \eta)}{|g(x)-(\xi, \eta)|} \text { for } x \in X, \\
& A=E, \quad B=N, \quad C=W \cup S .
\end{aligned}
$$


Lemma 8.8. If $A \in \Gamma, g \in C_{2}\left(A \cup A^{*}\right)$, and if $g \mid A$ is an essential map of $A$ into $E_{2}-\{z\}$, then $z$ is a stable value of $g$.

Proof. Assuming that

$$
0<r<\inf _{x \in A}|g(x)-z|
$$

we consider the cohomology diagram:

$$
H^{2}\left[E_{2}, E_{2}-K(z, r)\right] \stackrel{\delta}{\longleftarrow} H^{1}\left[E_{2}-K(z, r)\right]
$$

Since $A \cup A^{\#}$ does not separate $E_{2}$, the group $H^{1}\left(A \cup A^{\#}\right)$ and the homomorphism $i^{*}$ are trivial. By exactness $\delta^{\prime}$ is an isomorphism. From this and the fact that the homomorphism $(g \mid A)^{*}$ is nontrivial it follows that the homomorphism

$$
g^{*} \circ \delta=\delta^{\prime} \circ(g \mid A)^{*}
$$

is nontrivial. Hence $g^{*}$ is a nontrivial homomorphism.

Lemma 8.9. If $A \in \Gamma, B \in \Gamma, C \in \Gamma$,

$$
\begin{aligned}
& A \cup A^{\#} \subset B^{\#}, \quad C \subset\left(B \cup B^{*}\right)-A^{*}, \\
& g \in C_{2}\left[\left(B \cup B^{\#}\right)-A^{\#}\right], \\
& g \mid A \text { is an inessential map of } A \text { into } E_{2}-\{z\}, \\
& g \mid C \text { is an essential map of } C \text { into } E_{2}-\{z\},
\end{aligned}
$$

then $z$ is a stable value of $g$.

Proof. There is a mapping

$$
f \in C_{2}\left(B \cup B^{H}\right)
$$

such that

$$
f \mid\left[\left(B \cup B^{\#}\right)-A^{\#}\right]=g, \quad f\left(A \cup A^{\#}\right) \subset E_{2}-\{z\} .
$$

Noting that

$$
f|C=g| C, \quad C \cup C^{\sharp} \subset B \cup B^{\sharp},
$$

we infer that $z$ is a stable value of $f \mid\left(C \cup C^{\#}\right)$ and of $f$. Since

$$
\{x \mid f(x)=z\} \subset\left(B \cup B^{*}\right)-\left(A \cup A^{*}\right)
$$


and $\left(B \cup B^{\sharp}\right)-\left(A \cup A^{\sharp}\right)$ is open in $B \cup B^{\sharp}$, it follows that $z$ is a stable value of

$$
f\left|\left[\left(B \cup B^{\sharp}\right)-\left(A \cup A^{\sharp}\right)\right]=g\right|\left[\left(B \cup B^{\sharp}\right)-\left(A \cup A^{\sharp}\right)\right]
$$

and of $g$.

THEOREM 8.10. Suppose:

(1) $X$ is a locally connected, locally compact subset of $E_{2}$ for which $\sigma(X)>0$.

(2) $G \subset X$ and $G$ is open relative to $X$.

(3) $u \in C_{1}(G), v \in C_{1}(G), g \in C_{2}(G)$, and

$$
g(x)=(u(x), v(x)) \text { for } x \in G .
$$

(4) For each $z=\left(z_{1}, z_{2}\right) \in E_{2}, \nu(z)$ is the supremum of the set of all positive integers $m$ with the following property:

$G$ contains $m$ disjoint continua $A_{1}, A_{2}, \cdots, A_{m}$ such that

$$
\begin{gathered}
z_{1}=u(x) \text { for } x \in \bigcup_{i=1}^{m} A_{i}, \\
z_{2} \in \operatorname{Interior} v\left(A_{i}\right) \text { for } i=1,2, \cdots, m .
\end{gathered}
$$

Then:

(5) $\nu(z) \leqq S(g, G, z)$ for $\mathcal{L}_{2}$ almost all $z$ in $E_{2}$.

Proof. Let $m$ be a positive integer.

We choose points $p_{1}, p_{2}, p_{3}, \cdots$ which form a countable dense subset of $E_{2}$ and define

$$
P_{j}=\Omega \cap\left\{A \mid A \subset G \text { and } p_{j} \in A^{\sharp}\right\} \quad \text { for } j=1,2,3, \cdots .
$$

We also define

$$
P_{0}=\Omega \cap\left\{A \mid A \subset G \text { and } A^{\#}=0\right\} .
$$

Corresponding to each $m$-termed sequence

$$
q=\left(q_{1}, q_{2}, \cdots, q_{m}\right)
$$

of non-negative integers, and for any rational numbers $r<s$, we let

$$
F(q, r, s)
$$

be the set of all $m$-termed sequences

$$
A=\left(A_{1}, A_{2}, \cdots, A_{m}\right)
$$

with the following properties:

$$
A_{i} \in P_{q_{i}} \text { for } i=1,2, \cdots, m \text {; }
$$




$$
\begin{aligned}
& A_{1}, A_{2}, \cdots, A_{m} \text { are disjoint; } \\
& u \text { is constant on } \bigcup_{i=1}^{m} A_{i} ; \\
& \inf _{x \in A_{i}} v(x)<r<s<\sup _{x \in A_{i}} v(x) \text { for } i=1,2, \cdots, m .
\end{aligned}
$$

Setting

$$
U(A)=u(x) \text { whenever } A \in F(q, r, s) \text { and } x \in \bigcup_{i=1}^{m} A_{i},
$$

we observe that the set

$$
\{z \mid \nu(z) \geqq m\}
$$

is the union of the countably many cartesian products

$$
U[F(q, r, s)] \times\{\eta \mid r<\eta<s\} .
$$

In order to prove that

$$
S(g, G, z) \geqq m \text { for } \mathcal{L}_{2} \text { almost all } z \text { in }\{z \mid \nu(z) \geqq m\}
$$

it is therefore sufficient to verify the following statement:

For each of the above triples $(q, r, s)$ there is a set $\mu$ such that

$$
\begin{aligned}
& \mu \subset U[F(q, r, s)], \quad U[F(q, r, s)]-\mu \text { is countable, } \\
& S[g, G,(\xi, \eta)] \geqq m \text { whenever } \xi \in \mu \text { and } r<\eta<s .
\end{aligned}
$$

For the purpose of proving this we henceforth fix $q, r, s$ and abbreviate " $F(q, r, s)$ " by " $F$."

Associating with each $A \in F$ the $2 m$-termed sequence

$$
\left(A_{1}, A_{1} \cup A_{1}^{\#}, A_{2}, A_{2} \cup A_{2}^{\#}, \cdots, A_{m}, A_{m} \cup A_{m}^{\#}\right)
$$

we establish a one-to-one correspondence between $F$ and a subset of the $2 m$-fold cartesian product of $\Gamma$. Since $\Gamma$ is separably metrized by $\rho$, the set $F$ becomes a separable metric space with the distance

$$
\sum_{i=1}^{m} \rho\left(A_{i}, B_{i}\right)+\rho\left(A_{i} \cup A_{i}^{\#}, B_{i} \cup B_{i}^{*}\right)
$$

between $A \in F$ and $B \in F$.

We let $R$ be the set of those points of $F$ at which the function $U$ has either a relative maximum or a relative minimum, infer that

$$
U(R) \text { is countable, }
$$

and define 


$$
\mu=U(F)-U(R)
$$

Now suppose

$$
\xi \in \mu, \quad r<\eta<s, \quad \xi=U(A), \quad A \in F-R .
$$

We successively choose

$t, G_{1}, G_{2}, \cdots, G_{m}, t^{\prime}, V_{1}, V_{2}, \cdots, V_{m}, W_{1}, W_{2}, \cdots, W_{m}, t^{\prime \prime}, B, C$

in such a way that

$0<t<\sigma(X)$

$G_{i}=X \cap\left\{x\left|\inf _{y \in A_{i}}\right| x-y \mid<t\right\}$,

$G_{1}, G_{2}, \cdots, G_{m}$ are disjoint subsets of $G$,

$0<t^{\prime}<t$,

$\gamma\left(A_{i}, Z^{\#}\right)<t$ whenever $q_{i}=0$ and $\gamma\left(A_{i}, Z\right)<t^{\prime}$,

$V_{i}$ and $W_{i}$ are connected and open relative to $X$,

Closure $\left(V_{i} \cup W_{i}\right)$ is a compact subset of $G_{i}$,

$\gamma\left(A_{i}, V_{i} \cup W_{i}\right)<t^{\prime}$,

$V_{i} \cap A_{i}$ and $W_{i} \cap A_{i}$ are nonvacuous,

$v(x)<r$ for $x \in V_{i}, v(x)>s$ for $x \in W_{i}$,

$0<3 t^{\prime \prime}<t^{\prime}$,

$V_{i} \cap Z$ and $W_{i} \cap Z$ are nonvacuous whenever $Z \subset X$ and $\gamma\left(Z, A_{i}\right)$ $<t^{\prime \prime}$,

$B \in F, C \in F$,

$\sum_{i=1}^{m} \rho\left(A_{i}, B_{i}\right)+\rho\left(A_{i} \cup A_{i}^{\#}, B_{i} \cup B_{i}^{\#}\right)<t^{\prime \prime}$,

$\sum_{i=1}^{m} \rho\left(A_{i}, C_{i}\right)+\rho\left(A_{i} \cup A_{i}^{\#}, C_{i} \cup C_{i}^{\#}\right)<t^{\prime \prime}$,

$U(B)<\xi<U(C)$.

It follows for each integer $i$ between 1 and $m$ that $V_{i} \cap B_{i}, W_{i} \cap B_{i}$, $V_{i} \cap C_{i}, W_{i} \cap C_{i}$ are nonvacuous and that one of the following three alternatives holds:

(i) $q_{i}=0$,

(ii) $q_{i}>0$ and $B_{i} \cup B_{i}^{\#} \subset C_{i}^{\#}$,

(iii) $q_{i}>0$ and $C_{i} \cup C_{i}^{\#} \subset B_{i}^{\#}$.

In case (i) holds we define

$$
Z_{i}=B_{i} \cup C_{i} \cup \text { Closure }\left(V_{i} \cup W_{i}\right), \quad X_{i}=Z_{i} \cup Z_{i}^{*}
$$

and conclude that

$\gamma\left(A_{i}, Z_{i}\right)<t^{\prime}$,

$\gamma\left(X, X_{i}\right) \leqq \gamma\left(A_{i}, X_{i}\right)<t<\sigma(X)$,

Boundary $X_{i} \subset Z_{i} \subset X$,

$X_{i} \subset X, X_{i} \subset G_{i}$,

$g \mid Z_{i}$ is an essential map of $Z_{i}$ into $E_{2}-\{(\xi, \eta)\}$,

$(\xi, \eta)$ is a stable value of $g \mid X_{i}$, 
$S\left[g, G_{i},(\xi, \eta)\right] \geqq 1$.

In case (ii) holds we define

$$
X_{i}=\left(C_{i} \cup C_{i}^{\#}\right)-B_{i}^{*},
$$

choose continua $V_{\boldsymbol{i}}^{\prime}$ and $W_{\boldsymbol{i}}^{\prime}$ such that

$$
\begin{aligned}
& V_{i}^{\prime} \subset X_{i} \cap V_{i}, W_{i}^{\prime} \subset X_{i} \cap W_{i}, \\
& V_{i}^{\prime} \cap B_{i}, W_{i}^{\prime} \cap B_{i}, V_{i}^{\prime} \cap C_{i}, W_{i}^{\prime} \cap C_{i} \text { are nonvacuous, }
\end{aligned}
$$

let

$$
Z_{i}=B_{i} \cup C_{i} \cup V_{i}^{\prime} \cup W_{i}^{\prime},
$$

and conclude that

$$
\begin{aligned}
\gamma\left(X, X_{i}\right) & \leqq \gamma\left(A_{i}, X_{i}\right) \leqq \gamma\left(A_{i}, B_{i}\right)+\gamma\left(B_{i}, X_{i}\right) \\
& =\gamma\left(A_{i}, B_{i}\right)+\gamma\left(B_{i} \cup B_{i}^{\#}, X_{i}\right) \\
& \leqq \gamma\left(A_{i}, B_{i}\right)+\gamma\left(B_{i} \cup B_{i}^{\#}, C_{i} \cup C_{i}^{\#}\right)<3 t^{\prime \prime}<t^{\prime}<t<\sigma(X),
\end{aligned}
$$

Boundary $X_{i} \subset B_{i} \cup C_{i} \subset X$,

$X_{i} \subset X, X_{i} \subset G_{i}, Z_{i} \subset X_{i}$,

$g \mid Z_{i}$ is an essential map of $Z_{i}$ into $E_{2}-\{(\xi, \eta)\}$,

$g \mid B_{i}$ is an inessential map of $B_{i}$ into $E_{2}-\{(\xi, \eta)\}$,

$(\xi, \eta)$ is a stable value of $g \mid X_{i}$,

$S\left[g, G_{i},(\xi, \eta)\right] \geqq 1$.

Since the third alternative is quite similar to the second, we obtain the result that

$$
S\left[g, G_{i},(\xi, \eta)\right] \geqq 1 \text { for } i=1,2, \cdots, m
$$

whence it follows that

$$
S[g, G,(\xi, \eta)] \geqq \sum_{i=1}^{m} S\left[g, G_{i},(\xi, \eta)\right] \geqq m .
$$

The proof is complete.

Actually it is true that

$$
\nu(z)=S(g, G, z) \text { for } \mathcal{L}_{2} \text { almost all } z \text { in } E_{2}
$$

because $\nu(z) \geqq S(g, G, z)$ for all $z$ in $E_{2}$, but we shall neither prove nor use this fact here.

THEOREM 8.11. Suppose:

(1) $X$ is a locally connected, locally compact subset of $E_{2}$ for which $\sigma(X)>0$. 
(2) $G \subset X$ and $G$ is open relative to $X$.

(3) $u \in C_{1}(G), v \in C_{1}(G), w \in C_{1}(G), f \in C_{3}(G), g \in C_{2}(G), h \in C_{2}(G)$, and

$$
\begin{aligned}
& f(x)=(u(x), w(x), v(x)), g(x)=(u(x), v(x)), \\
& h(x)=(u(x), w(x)) \text { for } x \in G .
\end{aligned}
$$

(4) $\int_{E_{2}} S(g, G, z) d \mathcal{L}_{2} z<\infty$.

Then:

(5) $\mathcal{L}_{2}\left[l_{h}\left(\Gamma \cap \mathcal{X}_{h}-\mathcal{X}_{f}\right)\right]=0$.

Proof. The elements of the set

$$
\Gamma \cap \mathscr{X}_{h}-\mathscr{X}_{f}
$$

are continua on which the functions $h, u$, and $w$ are constant, but the functions $f$ and $v$ are not constant. The denial of (5) implies the existence of rational numbers $r<s$ and a Borel set $P$ of $\mathcal{X}_{h}$ such that

$$
\begin{gathered}
P \subset \mathscr{x}_{h} \cap \Gamma, \quad \mathcal{L}_{2}\left[l_{h}(P)\right]>0, \\
\inf _{x \in A} v(x)<r<s<\sup _{x \in A} v(x) \text { for } A \in P .
\end{gathered}
$$

Defining the function $\nu$ as in the preceding theorem we find that $\nu(\xi, \eta) \geqq$ (number of elements of $\left\{\zeta \mid(\xi, \zeta) \in l_{h}(P)\right\}$ ) for $r<\eta<s$,

$$
\begin{aligned}
\infty & >\int_{E_{2}} S(g, G, z) d \mathcal{L}_{{ }_{2}} z \geqq \int_{E_{2}} \nu(z) d \mathcal{L}_{2} z \geqq \int_{-\infty}^{\infty} \int_{r}^{s} \nu(\xi, \eta) d \mathcal{L}_{1} \eta d \mathcal{L}_{1} \xi \\
& \left.\geqq(s-r) \cdot \int_{-\infty}^{\infty} \text { (number of elements of }\left\{\zeta \mid(\xi, \zeta) \in l_{h}(P)\right\}\right) d \mathcal{L}_{1} \xi, \\
& \left\{\zeta \mid(\xi, \zeta) \in l_{h}(P)\right\} \text { is finite for } \mathcal{L}_{1} \text { almost all } \xi \text { in } E_{1}, \\
0 & =\int_{-\infty}^{\infty} \mathcal{L}_{1}\left(\left\{\zeta \mid(\xi, \zeta) \in l_{h}(P)\right\}\right) d \mathcal{L}_{1} \xi=\mathcal{L}_{2}\left[l_{h}(P)\right]>0 .
\end{aligned}
$$

For the special case in which $X=G$ is a 2 -cell the preceding Theorem 8.11 was proved by L. Cesari in [CE 2]. He used it in showing that $L_{2}(f)$ does not exceed the sum of the Lebesgue areas of the three orthogonal projections of $f$ onto the coordinate planes.

THEOREM 8.12. Suppose:

(1) $X$ is a locally connected, locally compact subset of $E_{2}$ for which $\sigma(X)>0$.

(2) $G \subset X$ and $G$ is open in $X$.

(3) $f \in C_{n}(X), n \geqq 3$, and $\lambda_{2}(f, G)<\infty$. 
(4) $R \in G_{n}$ and $h=p_{n}^{2} \circ R \circ f$.

Then:

(5) $\mathcal{L}_{2}\left[l_{h}\left(\Gamma \cap \mathscr{X}_{h}-\mathscr{X}_{f}\right)\right]=0$.

Proof. Defining the functions

$$
\begin{gathered}
u, w, v_{1}, \cdots, v_{n-2} \in C_{1}(G) ; \\
g_{1}, \cdots, g_{n-2} \in C_{2}(G) ; \quad f_{1}, \cdots, f_{n-2} \in C_{3}(G)
\end{gathered}
$$

so that

$$
\begin{aligned}
(R \circ f)(x) & =\left(u(x), w(x), v_{1}(x), \cdots, v_{n-2}(x)\right), \\
g_{j}(x) & =\left(u(x), v_{j}(x)\right), \quad f_{j}(x)=\left(u(x), w(x), v_{j}(x)\right)
\end{aligned}
$$

for $x \in G$ and $j=1, \cdots, n-2$, we conclude that

$$
\begin{gathered}
h(x)=(u(x), w(x)) \text { for } x \in G, \\
\int_{E_{2}} S\left(g_{j}, G, z\right) d \mathcal{L}_{2} z \leqq \lambda_{2}\left(g_{j}, G\right) \leqq \lambda_{2}(f, G)<\infty \text { for } j=1, \cdots, n-2, \\
\Gamma \cap \mathcal{X}_{h}-\mathcal{X}_{f} \subset \bigcup_{j=1}^{n-2} \Gamma \cap \mathcal{X}_{h}-\mathcal{X}_{f_{j},} \\
\mathcal{L}_{2}\left[l_{h}\left(\Gamma \cap \mathcal{X}_{h}-\mathcal{X}_{f}\right)\right] \leqq \sum_{j=1}^{n-2} \mathcal{L}_{2}\left[l_{h}\left(\Gamma \cap \mathcal{X}_{h}-\mathcal{X}_{f_{j}}\right)\right]=0 .
\end{gathered}
$$

Theorem 8.13. Suppose:

(1) $X$ is a locally connected, locally compact subset of $E_{2}$ for which $\sigma(X)>0$.

(2) $G \subset X$ and $G$ is open in $X$.

(3) $f \in C_{2}(G)$ and $\lambda_{2}(f, G)<\infty$.

(4) $P=\mathscr{H}_{f} \cap\left\{A \mid A^{\#} \neq 0\right.$ and $\left.\lim \sup _{r \rightarrow 0+} D\left[f, r, \Delta_{f}(A, r)\right]>0\right\}$. Then:

(5) $\mathcal{L}_{2}\left[l_{f}(P)\right]=0$.

Proof. It is sufficient to consider a point $q \in E_{2}$ and a set

$$
Q \subset P \cap\left\{A \mid q \in A^{\#} \text { and } S\left[f, G, l_{f}(A)\right]<\infty\right\}
$$

such that $l_{f}$ is univalent on $Q$, and to prove that $Q$ is countable.

The subset $Q$ of $\Omega$ is a separable metric space with the distance

$$
\rho(A, B)+\rho\left(A \cup A^{\sharp}, B \cup B^{\sharp}\right)
$$

between $A \in Q$ and $B \in Q$. Furthermore $Q$ is simply ordered by the relation

$$
(Q \times Q) \cap\left\{(A, B) \mid A \subset B^{*}\right\} .
$$


We recall that any function on a separable metric space into an ordered set has only countably many relative maximum or minimum values. Applying this principle to the identity map of $Q$ we find that only countably many points of $Q$ are isolated on the right or left. Hence $Q$ has a countable subset $Q^{\prime}$ with the following property:

If $A \in Q-Q^{\prime}$, then there exist sequences

$$
B_{1}, B_{2}, B_{3}, \cdots ; \quad C_{1}, C_{2}, C_{3}, \cdots
$$

of elements of $Q$ such that

$$
\begin{aligned}
& B_{j} \subset A^{\#}, \quad A \subset C_{j}^{\#} \text { for } j=1,2,3, \cdots, \\
& \lim _{j \rightarrow \infty} \rho\left(A, B_{j}\right)+\rho\left(A \cup A^{\#}, B_{j} \cup B_{j}^{\#}\right)=0, \\
& \lim _{j \rightarrow \infty} \rho\left(A, C_{j}\right)+\rho\left(A \cup A^{\#}, C_{j} \cup C_{j}^{\#}\right)=0 .
\end{aligned}
$$

We shall prove that this property of $A$ violates our assumptions about $Q$, thus showing that $Q=Q^{\prime}$ and $Q$ is countable.

The sets

$$
X_{j}=\left(C_{j} \cup C_{j}^{\#}\right)-B_{j}^{\#}
$$

are continua for which

$$
\begin{aligned}
& A \subset \text { Interior } X_{j}, \text { Boundary } X_{j} \subset B_{j} \cup C_{j} \subset G \subset X, \\
& \gamma\left(A, X_{j}\right) \leqq \gamma\left(A, B_{j}\right)+\gamma\left(B_{j} \cup B_{j}^{\#}, C_{j} \cup C_{j}^{\#},\right. \\
& \lim _{j \rightarrow 0} \gamma\left(A, X_{j}\right)=0<\sigma(X), \\
& X_{j} \subset G \text { for large } j, \\
& \lim _{j \rightarrow \infty} \sup _{x \in X_{j}} d_{f}\left[A, m_{f}(x)\right]=0 .
\end{aligned}
$$

There are positive numbers $r_{1}, r_{2}, r_{3}, \cdots$ such that

$$
\begin{gathered}
\lim _{j \rightarrow \infty} r_{j}=0, \\
D\left[f, r_{j}, \Delta_{f}\left(A, r_{j}\right)\right]>0 \quad \text { for } j=1,2,3, \cdots .
\end{gathered}
$$

Passing to subsequences we may assume that

$$
\begin{gathered}
G \supset X_{j} \supset \Delta_{f}\left(A, r_{j}\right) \supset X_{i+1}, \\
f\left(\text { Boundary } X_{j}\right) \subset\left\{l_{f}\left(B_{j}\right), l_{f}\left(C_{j}\right)\right\} \subset E_{2}-K\left[l_{f}(A), r_{i}\right]
\end{gathered}
$$

for every positive integer $j$.

Now consider the cohomology diagram: 


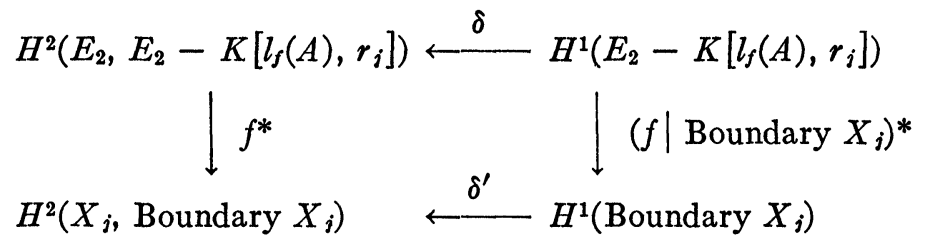

Since $f$ (Boundary $X_{j}$ ) is a finite set, the homomorphism $\left(f \mid\right.$ Boundary $\left.X_{j}\right) *$ is trivial. It follows that the homomorphism

$$
f^{*} \circ \delta=\delta^{\prime} \circ\left(f \mid \text { Boundary } X_{j}\right)^{*}
$$

is trivial. Furthermore $\delta$ is an isomorphism onto the domain of $f^{*}$. Hence $f^{*}$ is a trivial homomorphism.

We conclude that $X_{j}$ contains an essential canonical region $Z_{j}$ of $\left(f, G, l_{f}(A), r_{j}\right)$ other than $\Delta_{f}\left(A, r_{j}\right)$. Then the sets $Z_{1}, Z_{2}, Z_{3}, \cdots$ are disjoint and

$$
S\left[f, G, l_{f}(A)\right]=\infty .
$$

TheOREM 8.14. Suppose:

(1) $X$ is a locally connected, locally compact subset of $E_{2}$ for which $\sigma(X)>0$.

(2) $f \in C_{n}(X)$ and $n \geqq 3$.

(3) $V_{1}, V_{2}, V_{3}, \cdots$ and $W$ are open subsets of $\mathcal{X}_{f}$.

(4) $U_{i}=\left\{x \mid m_{f}(x) \in V_{i}\right\}$ and $\lambda_{2}\left(f, U_{i}\right)<\infty$ for $i=1,2,3, \cdots$.

(5) $\bigcup_{i=1}^{\infty}\left(V_{i} \cap \Gamma \cap\left\{A \mid A^{\#}=0\right.\right.$ and $A \subset$ Interior $\left.\left.U_{i}\right\}\right) \subset W$.

(6) $R \in G_{n}$ and $h=p_{n}^{2} \circ R \circ f \mid \bigcup_{i=1}^{\infty} U_{i}$.

\section{Then:}

(7) $\mathcal{L}_{2}\left[l_{h}\left(\Gamma \cap \mathscr{X}_{h}-\mathscr{X}_{f}\right)\right]=0$.

(8) $S\left(h, \cup_{i=1}^{\infty} U_{i}, z\right) \leqq S\left(h,\{x \mid f(x) \in W\}\right.$, z) for $\mathcal{L}_{2}$ almost all $z$ in $E_{2}$.

(9) $S\left(h, \cup_{i=1}^{\infty} U_{i}, z\right) \leqq \sum_{i=1}^{\infty} S\left(h, U_{i}, z\right)$ for $\mathcal{L}_{2}$ almost all $z$ in $E_{2}$.

Proof of (7). We define

$$
Q=\bigcup_{i=1}^{\infty} l_{\left(h \mid U_{i}\right)}\left[\Gamma \cap \mathcal{X}_{\left(h \mid U_{i}\right)}-\mathcal{X}_{\left(f \mid U_{i}\right)}\right],
$$

note that

$$
\mathcal{L}_{2}(Q)=0,
$$

and shall complete the proof by verifying that

$$
l_{h}\left[\Gamma \cap \mathscr{X}_{h}-\mathscr{X}_{f}\right] \subset Q .
$$

We suppose

$$
A \in \Gamma \cap \mathscr{X}_{h}-\mathscr{X}_{f}
$$


note that $m_{f}(A)$ is a compact subset of

$$
\bigcup_{i=1}^{\infty} V_{i}
$$

and choose closed sets $F_{i} \subset V_{i}$ such that

$$
m_{f}(A) \subset \bigcup_{i=1}^{\infty} F_{i} \text {. }
$$

Then

$$
m_{f}(A)=\bigcup_{i=1}^{\infty}\left[F_{i} \cap m_{f}(A)\right],
$$

$m_{f}(A)$ is a nondegenerate continuum,

$m_{f}(A)$ is not zero-dimensional,

and there exists a positive integer $i$ such that

$F_{i} \cap m_{f}(A)$ is not zero-dimensional,

$F_{i} \cap m_{f}(A)$ is not totally disconnected.

Choosing a nondegenerate component $B$ of $F_{i} \cap m_{f}(A)$ and a set $C$ such that

$$
C \in \mathcal{H}_{\left(h \mid U_{i}\right)},\left\{x \mid m_{f}(x) \in B\right\} \subset C \subset A,
$$

we conclude that

$$
\begin{aligned}
C & \in \Gamma \cap \mathscr{X}_{\left(h \mid U_{i}\right)}-\mathscr{X}_{\left(f \mid U_{i}\right)}, \\
l_{h}(A) & =l_{\left(h \mid U_{i}\right)}(C) \in Q .
\end{aligned}
$$

Proof of (8) ANd (9). We define

$$
P_{i}=\mathcal{X}_{h} \cap\left\{A \mid A \subset U_{i}, A^{\#} \neq 0 \text {, and } \limsup _{t \rightarrow 0+} D\left[h, t, \Delta_{h}(A, t)\right]>0\right\}
$$

for $i=1,2,3, \cdots$ and infer that

$$
\mathcal{L}_{2}\left(l_{h}\left[\left(\Gamma \cap \mathscr{X}_{h}-\mathscr{X}_{f}\right) \cup \bigcup_{i=1}^{\infty} P_{i}\right]\right)=0 .
$$

Now suppose

$$
z \in E_{2}-l_{h}\left[\left(\Gamma \cap \mathcal{X}_{h}-\mathcal{X}_{f}\right) \cup \bigcup_{i=1}^{\infty} P_{i}\right]
$$

and $Z$ is an essential canonical region of $\left(h, \bigcup_{i=1}^{\infty} U_{i}, z, r\right)$. 
According to Theorem 6.10 there is a set $A$ such that

$$
\begin{gathered}
A \in \Gamma \cap \mathcal{X}_{h}, \quad l_{h}(A)=z, \quad \Delta_{h}(A, r)=Z, \\
\limsup _{t \rightarrow 0^{+}} D\left[h, t, \Delta_{h}(A, t)\right]>0,
\end{gathered}
$$

It follows that

$A \in \mathcal{X}_{f}, A \subset$ Interior $U_{i}$ for some positive integer $i$, $A^{\sharp}=0, \quad A \subset\left\{x \mid m_{f}(x) \in W\right\}$

and we can choose $t$ so that

$$
\begin{aligned}
& 0<t<r, \quad D\left[h, t, \Delta_{h}(A, t)\right]>0, \\
& \Delta_{h}(A, t) \subset U_{i} \cap\left\{x \mid m_{f}(x) \in W\right\} .
\end{aligned}
$$

Hence $Z$ contains the essential canonical region $\Delta_{h}(A, t)$ of $\left(h \mid U_{i}, U_{i}, z, t\right)$ and of $\left(h \mid\left\{x \mid m_{f}(x) \in W\right\},\left\{x \mid m_{f}(x) \in W\right\}, z, t\right)$.

TheOREM 8.15. Suppose:

(1) $X$ is a 2-cell.

(2) $f \in C_{n}(X)$ and $L_{2}(f)<\infty$.

\section{Then:}

(3) $L_{2}(f) \leqq H_{f}^{2}\left[\left(\mathscr{X}_{f}-\mathscr{X}_{f}^{*}\right) \cap\left\{A \mid \varepsilon_{2}(f, A) \geqq 1\right\}\right]$.

(4) $L_{2}(f)=H_{f}^{2}\left[\left(\mathcal{X}_{f}-\mathcal{X}_{f}^{*}\right) \cap\left\{A \mid \varepsilon_{2}(f, A)=1\right\}\right]$.

(5) $L_{2}^{*}(f, A)=L_{* 2}(f, A)=\varepsilon_{2}(f, A)=0$ or 1 for $H_{f}^{2}$ almost all $A$ in $\mathcal{J l}_{f}$.

(6) The stable area

$$
\int_{E_{2}} S\left(p_{n}^{2} \circ R \circ f, X, z\right) d \mathcal{L}_{2} z
$$

of the projection $p_{n}^{2} \circ R \circ f$ of $f$ is continuous with respect to $R$ on $G_{n}$.

(7) If $R \in G_{n}$, then

$$
S\left(p_{n}^{2} \circ R \circ f, X, z\right)=N\left(p_{n}^{2} \circ R \circ l_{f},\left\{A \mid \varepsilon_{2}(f, A)=1\right\}, z\right)
$$

for $\mathcal{L}_{2}$ almost all $z$ in $E_{2}$.

(8) If $R \in G_{n}$ and $W$ is an open subset of $\mathcal{X C}_{f}$, then

$$
\begin{aligned}
S\left(p_{n}^{2} \circ R \circ f,\left\{x \mid m_{f}(x)\right.\right. & \in W\}, z) \\
& =N\left(p_{n}^{2} \circ R \circ l_{f}, W \cap\left\{A \mid \varepsilon_{2}(f, A)=1\right\}, z\right)
\end{aligned}
$$

for $\mathcal{L}_{2}$ almost all $z$ in $E_{2}$.

Proof of (3) AND (6). The properties (3) and (6) of $f$ are Fréchet 
invariant as well as both cyclically and internally extensive. Hence we need only consider the special case in which $f$ is a nondegenerate mapping whose partial derivatives $D_{1} f(x)$ and $D_{2} f(x)$ exist for $\mathcal{L}_{2}$ almost all $x$ in $X-\dot{X}$ and whose Lebesgue area $L_{2}(f)$ equals the Dirichlet integral

$$
\frac{1}{2} \int_{X-\dot{x}}\left|D_{1} f(x)\right|^{2}+\left|D_{2} f(x)\right|^{2} d \mathcal{L}_{2} x
$$

These assumptions imply that

$$
\begin{gathered}
\left|D_{1} f(x)\right|=\left|D_{2} f(x)\right|=(J f(x))^{1 / 2}, \\
D_{1} f(x) \bullet D_{2} f(x)=0
\end{gathered}
$$

for $\mathcal{L}_{2}$ almost all $x$ in $X-\dot{X}$. Furthermore

$$
\int_{E_{2}} S\left(p_{n}^{2} \circ R \circ f, X, z\right) d \mathcal{L}_{2} z=\int_{X-\dot{x}} J\left(p_{n}^{2} \circ R \circ f\right)(x) d \mathcal{L}_{2}(x)
$$

for $R \in G_{n}$, and for each $x \in X-\dot{X}$ such that $D_{1} f(x)$ and $D_{2} f(x)$ exist the Jacobian

$$
J\left(p_{n}^{2} \circ R \circ f\right)(x)
$$

is continuous with respect to $R$ and does not exceed $J f(x)$.

Now (6) follows immediately.

In order to prove (3) we define

$$
P=(X-\dot{X}) \cap\{x \mid J f(x)>0\}
$$

and infer that the following ten conditions are satisfied for $\mathcal{L}_{2}$ almost all points $x$ of $P$ :

(i) $m_{f}(x)=\{x\} \in \mathscr{X}_{f}-\mathscr{X}_{f}$.

(ii) $\lim _{r \rightarrow 0+} \mathcal{L}_{2}\left[K(x, r)-\Delta_{f}\left(x, r(J f(x))^{1 / 2}\right)\right] /\left(\pi r^{2}\right)=0$.

(iii) There is a continuously differentiable mapping $g \in C_{n}\left(E_{2}\right)$ and a compact set $G$ such that $\lim _{r \rightarrow 0+} \mathcal{L}_{2}[K(x, r)-G] /\left(\pi r^{2}\right)=0$ and

$$
x \in G \subset\left\{z \mid g(z)=f(z), D_{1} g(z)=D_{1} f(z), D_{2} g(z)=D_{2} f(z)\right\} .
$$

(iv) There is an $R \in G_{n}$ such that

$$
J f(x)=J g(x)=J h(x), \text { where } h=p_{n}^{2} \circ R \circ f .
$$

(v) $\lim _{r \rightarrow 0+} \mathcal{L}_{2}\left(\left[p_{n}^{2} \circ R \circ g\right][K(x, r)]\right) /\left(\pi r^{2}\right)=J f(x)$.

(vi) $\lim _{r \rightarrow 0+} \mathcal{L}_{2}\left(h\left[H \cap \Delta_{f}\left(x, r(J f(x))^{1 / 2}\right)\right]\right) /\left(\pi r^{2}\right) \geqq J f(x)$, where $H=G \cap\{z \mid J h(z)>0\}$.

(vii) $\lim _{r \rightarrow 0+} \mathcal{L}_{2}\left(h\left[H \cap \Delta_{f}(x, r)\right]\right) /\left(\pi r^{2}\right) \geqq 1$.

(viii) $\mathcal{L}_{2}\left(h\left[H \cap \Delta_{f}(x, r)\right]-\left\{z \mid M\left[h, \Delta_{f}(x, r), z\right] \geqq 1\right\}\right)=0$ for $r>0$. 
(ix) $p_{n}^{2} \circ R$ is a 1 -fold essential projection for $f$ at $\{x\}$.

(x) $\varepsilon_{2}(f,\{x\}) \geqq 1$.

Certain techniques developed in [R 2], [F 1], and [W] are helpful in checking the statements (i), (ii), (iii), and (viii).

We let

$$
Q=P \cap\left\{x \mid\{x\} \in \mathscr{X}_{f}-\mathscr{X}_{f} \text { and } \varepsilon_{2}(f,\{x\}) \geqq 1\right\}
$$

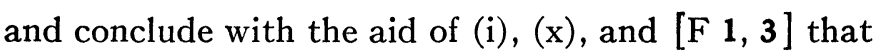

$$
\begin{aligned}
H_{f}^{2}\left[\left(\mathcal{X}_{f}-\mathscr{X}_{f}\right) \cap\{A \mid\right. & \left.\left.\mathcal{E}_{2}(f, A) \geqq 1\right\}\right] \\
& \geqq H_{f}^{2}\left[m_{f}(Q)\right] \geqq \int_{E_{n}} N\left(l_{f}, m_{f}(Q), y\right) d \mathcal{F}_{n}^{2} y \\
& =\int_{E_{n}} N(f, Q, y) d \mathcal{K}_{n}^{2} y=\int_{Q} J f(x) d \mathcal{L}_{2} x \\
& =\int_{X-\dot{x}} J f(x) d \mathcal{L}_{2} x=L_{2}(f) .
\end{aligned}
$$

Proof of (4) AND (5). We know from Theorem 6.7 that

$$
L_{2}^{*}(f, A) \geqq L_{* 2}(f, A) \geqq \varepsilon_{2}(f, A) \text { for } A \in \mathcal{X}_{f}
$$

and infer with the help of Theorem 6.2 and (3) that

$$
\begin{aligned}
\infty>L_{2}(f) & \geqq \int_{\mathcal{X}_{f}} L_{2}^{*}(f, A) d H_{f}^{2} A \geqq \int_{\mathcal{X}_{f}} L_{* 2}(f, A) d H_{f}^{2} A \\
& \geqq \int_{\mathcal{X}_{f}} \varepsilon_{2}(f, A) d H_{f}^{2} A \geqq \int_{\mathcal{X}_{f}-\mathcal{X}_{j}} E_{2}(f, A) d H_{f}^{2} A \\
& =\sum_{i=1}^{\infty} H_{f}^{2}\left[\left(\mathcal{X}_{f}-\mathcal{X}_{f}^{*}\right) \cap\left\{A \mid \varepsilon_{2}(f, A) \geqq i\right\}\right] \\
& \geqq L_{2}(f)+H_{f}^{2}\left[\left(\mathcal{X}_{f}-\mathcal{X}_{f}\right) \cap\left\{A \mid \varepsilon_{2}(f, A) \geqq 2\right\}\right] .
\end{aligned}
$$

Proof of (7). From (4), (5), and Theorem 6.6 we know that

$$
N\left(p_{n}^{2} \circ R \circ l_{f}, \quad\left\{A \mid \varepsilon_{2}(f, A)=1\right\}, z\right) \geqq S\left(p_{n}^{2} \circ R \circ f, X, z\right)
$$

for $\mathcal{L}_{2}$ almost all $z$ in $E_{2}$ whenever $R \in G_{n}$.

We define the functions $u$ and $v$ on $G_{n}$ such that

$$
u(R)=\int_{E n} N\left(p_{n}^{2} \circ R \circ l_{f},\left\{A \mid \mathcal{E}_{2}(f, A)=1\right\}, z\right) d \mathcal{L}_{2} z,
$$




$$
v(R)=\int_{E_{2}} S\left(p_{n}^{2} \circ R \circ f, X, z\right) d \mathcal{L}_{2} z
$$

for $R \in G_{n}$, and shall complete the proof by showing that $u$ and $v$ are equal.

The function $v$ is continuous on $G_{n}$ by virtue of (6).

From (4), (5), and Theorem 6.9 we infer that each of the sets

$$
Y_{i}=\left\{y \mid N\left(l_{f},\left\{A \mid \varepsilon_{2}(f, A)=1\right\}, y\right) \geqq i\right\}
$$

is a Hausdorff 2 rectifiable subset of $E_{n}$. Since

$$
u(R)=\sum_{i=1}^{\infty} \int_{E_{2}} N\left(p_{n}^{2} \circ R, Y_{i}, z\right) d \mathcal{L}_{2} z \text { for } R \in G_{n},
$$

we conclude that the function $u$ is continuous on $G_{n}$.

Thus $u$ and $v$ are continuous functions on $G_{n}$ such that

$$
u(R) \geqq v(R) \text { for } R \in G_{n}
$$

and

$$
\begin{aligned}
\int_{G_{n}} u(R) d \phi_{n} R & =\beta(n, 2) \cdot \sum_{i=1}^{\infty} \mathcal{F}_{n}^{2}\left(Y_{i}\right)=\beta(n, 2) \cdot \sum_{i=1}^{\infty} \mathcal{F}_{n}^{2}\left(Y_{i}\right) \\
& =\beta(n, 2) \cdot \int_{E_{n}} N\left(l_{f},\left\{A \mid \mathcal{E}_{2}(f, A)=1\right\}, y\right) d \mathcal{C}_{n}^{2} y \\
& =\beta(n, 2) \cdot H_{f}^{2}\left(\left\{A \mid \mathcal{E}_{2}(f, A)=1\right\}\right)=\beta(n, 2) \cdot L_{2}(f) \\
& =\int_{G_{n}} v(R) d \phi_{n} R .
\end{aligned}
$$

It follows that $u=v$.

Proof of (8). Let $V_{1} \supset V_{2} \supset V_{3} \supset \cdots$ be such open subsets of $\mathcal{X}_{f}$ that

$$
\mathfrak{x}_{f}-W=\bigcap_{i=1}^{\infty} V_{i}
$$

and let

$$
\begin{aligned}
V_{i}^{\prime} & =\left\{x \mid m_{f}(x) \in V_{i}\right\} \quad \text { for } i=1,2,3, \cdots, \\
W^{\prime} & =\left\{x \mid m_{f}(x) \in W\right\}, \quad Q=\left\{A \mid \varepsilon_{2}(f, A)=1\right\}, \\
g & =p_{n}^{2} \circ R \circ l_{f}, \quad h=p_{n}^{2} \circ R \circ f .
\end{aligned}
$$

From (3), (4), and Theorem 6.6 as well as (7) and Theorem 8.14 
we infer for $\mathcal{L}_{2}$ almost all $z$ in $E_{2}$ that

$$
\begin{aligned}
N(g, Q, z) & <\infty, \\
S\left(h, W^{\prime}, z\right) & \leqq N(g, Q \cap W, z), \\
S\left(h, V_{i}^{\prime}, z\right) & \leqq N\left(g, Q \cap V_{i}, z\right), \\
N(g, Q, z)=S(h, X, z) & \leqq S\left(h, W^{\prime}, z\right)+S\left(h, V_{i}^{\prime}, z\right)
\end{aligned}
$$

for every positive integer $i$. For each such point $z$ there exists a positive integer $i$ such that

$$
N(g, Q \cap W, z)+N\left(g, Q \cap V_{i}, z\right)=N(g, Q, z),
$$

whence it follows that

$$
S\left(h, W^{\prime}, z\right)=N(g, Q \cap W, z) .
$$

Theorem 8.16. Suppose:

(1) $X$ is a locally compact, locally connected subset of $E_{2}$ for which $\sigma(X)>0$.

(2) $f \in C_{n}(X)$ and $n \geqq 3$.

(3) $U$ is the union of all those open subsets $V$ of $\mathcal{X l}_{f}$ for which

$$
\lambda_{2}\left(f,\left\{x \mid m_{f}(x) \in V\right\}\right)<\infty .
$$

Then:

(4) $Q=\mathscr{X}_{f} \cap\left\{A \mid L_{2}^{*}(f, A)=\varepsilon_{2}(f, A)=1\right\}$.

(5) If $W$ is an open subset of $U$ and $R \in G_{n}$, then

$$
S\left(p_{n}^{2} \circ R \circ f,\left\{x \mid m_{f}(x) \in W\right\}, z\right) \leqq N\left(p_{n}^{2} \circ R \circ l_{f}, W \cap Q, z\right)
$$

for $\mathcal{L}_{2}$ almost all $z$ in $E_{2}$.

(6) If $W$ is an open subset of $U$, then the 2-dimensional integralgeometric stable area of the mapping

$$
f \mid\left\{x \mid m_{f}(x) \in W\right\}
$$

does not exceed

$$
H_{f}^{2}(W \cap Q) .
$$

(7) If $W$ is an open subset of $\mathcal{X l}_{f}$ and $R \in G_{n}$, then

$$
S\left(p_{n}^{2} \circ R \circ f,\left\{x \mid m_{f}(x) \in W\right\}, z\right) \leqq \sum_{A \in W,\left(p_{n}^{2} \circ R \circ l_{f}\right)(A)=z} L_{* 2}(f, A)
$$

for $\mathcal{L}_{2}$ almost all $z$ in $E_{2}$.

(8) If $W$ is an open subset of $\mathcal{M}_{f}$, then the 2-dimensional integralgeometric stable area of the mapping 


$$
f \mid\left\{x \mid m_{f}(x) \in W\right\}
$$

does not exceed

$$
\int_{E_{n}} \sum_{A \in W, l f(A)=y} L_{* 2}(f, A) d \mathscr{F}_{n}^{2} y .
$$

Proof of (5). We choose $\epsilon>0$ and define

$$
g=p_{n}^{2} \circ R \circ l_{f}, \quad h=p_{n}^{2} \circ R \circ f .
$$

Furthermore we let $F$ be the set of all ordered pairs

$$
(V, T)
$$

such that $V$ is an open subset of $W, T$ is a two-cell contained in $X$, and

$$
\left\{x \mid m_{f}(x) \in V\right\} \subset \text { Interior } T, \quad L_{2}(f \mid T)<\infty .
$$

If $(V, T) \in F$, then $V$ is an open subset of $\mathcal{X}_{\left(f \mid T^{T}\right)}$,

$$
\begin{gathered}
\left\{x \mid m_{f}(x) \in V\right\}=\left\{x \mid m_{(f \mid T)}(x) \in V\right\}, \\
L_{2}^{*}(f, A)=L_{2}^{*}(f \mid T, A) \quad \text { for } A \in V, \\
\varepsilon_{2}(f, A)=\varepsilon_{2}(f \mid T, A) \quad \text { for } A \in V,
\end{gathered}
$$

$S\left(h,\left\{x \mid m_{f}(x) \in V\right\}, z\right)=N(g, V \cap Q, z)$ for $\mathcal{L}_{2}$ almost all z in $E_{2}$,

$$
H_{f}^{2}(V \cap Q) \leqq \lambda_{2}\left(f,\left\{x \mid m_{f}(x) \in V\right\}\right)<\infty .
$$

Defining

$$
W^{\prime}=\underset{(V, T) \in \in_{F}}{U} V
$$

we note that

$$
\left(W \cap \Gamma \cap\left\{A \mid A^{\#}=0 \text { and } A \subset \text { Interior } X\right)\right\} \subset W^{\prime},
$$

whence, by Theorem 8.14 ,

$$
S\left(h,\left\{x \mid m_{f}(x) \in W\right\}, z\right)=S\left(h,\left\{x \mid m_{f}(x) \in W^{\prime}\right\}, z\right)
$$

for $\mathcal{L}_{2}$ almost all $z$ in $E_{2}$, because there are open subsets $W_{1}, W_{2}, W_{3}, \cdots$ of $W$ such that

$$
\begin{gathered}
W=\bigcup_{i=1}^{\infty} W_{i}, \\
\lambda_{2}\left(f,\left\{x \mid m_{f}(x) \in W_{i}\right\}\right)<\infty \text { for } i=1,2,3, \cdots .
\end{gathered}
$$


Finally we choose pairs $\left(V_{1}, T_{1}\right),\left(V_{2}, T_{2}\right),\left(V_{3}, T_{3}\right), \ldots$ in $F$ such that

$$
\begin{gathered}
W^{\prime}=\bigcup_{i=1}^{\infty} V_{i}, \\
H_{f}^{2}\left(Q \cap V_{i+1} \cap \bigcup_{j=1}^{i} V_{j}\right) \leqq \epsilon 2^{-i} .
\end{gathered}
$$

It follows from the Theorems 8.14 and 8.15 that

$$
\begin{aligned}
S\left(h,\left\{x \mid m_{f}(x) \in W^{\prime}\right\}, z\right) & \leqq \sum_{i=1}^{\infty} S\left(h,\left\{x \mid m_{f}(x) \in V_{i}\right\}, z\right) \\
& =\sum_{i=1}^{\infty} N\left(g, V_{i} \cap Q, z\right)
\end{aligned}
$$

for $\mathcal{L}_{2}$ almost all $z$ in $E_{2}$.

However

$$
\begin{aligned}
\mathcal{L}_{2}\left(g\left[\underset{i \neq j}{\cup}\left(Q \cap V_{i} \cap V_{j}\right)\right]\right) & \leqq H_{f}^{2}\left[\underset{i \neq j}{U}\left(Q \cap V_{i} \cap V_{j}\right)\right] \\
& \leqq \sum_{i=1}^{\infty} H_{f}^{2}\left(Q \cap V_{i+1} \cap \bigcup_{j=1}^{i} V_{i}\right) \\
& \leqq \sum_{i=1}^{\infty} \epsilon 2^{-i}=\epsilon
\end{aligned}
$$

and

$$
\sum_{i=1}^{\infty} N\left(g, V_{i} \cap Q, z\right)=N\left(g, Q \cap W^{\prime}, z\right) \leqq N(g, Q \cap W, z)
$$

whenever

$$
z \in E_{2}-g\left[\bigcup_{i \neq j}\left(Q \cap V_{i} \cap V_{j}\right)\right] .
$$

We conclude that

$$
\mathcal{L}_{2}\left(\left\{z \mid S\left(h,\left\{x \mid m_{f}(x) \in W\right\}, z\right)>N(g, Q \cap W, z)\right\}\right) \leqq \epsilon .
$$

Proof of (6). We define

$$
Y_{i}=E_{n} \cap\left\{y \mid N\left(l_{f}, W \cap Q, y\right) \geqq i\right\} \quad \text { for } i=1,2,3, \cdots
$$

and compute: 


$$
\begin{aligned}
H_{f}^{2}(W & \cap Q) \geqq \int_{E_{n}} N\left(l_{f}, W \cap Q, y\right) d \mathcal{F}_{n}^{2} y \\
& =\sum_{i=1}^{\infty} \mathcal{F C}_{n}^{2}\left(Y_{i}\right) \geqq \sum_{i=1}^{\infty} \mathcal{F}_{n}^{2}\left(Y_{i}\right) \\
& =\sum_{i=1}^{\infty} \beta(n, 2)^{-1} \int_{G_{n}} \int_{E_{2}} N\left(p_{n}^{2} \circ R, Y_{i}, z\right) d \mathcal{L}_{2} z d \phi_{n} R \\
& =\beta(n, 2)^{-1} \int_{G_{n}} \int_{E_{2}} \sum_{i=1}^{\infty} N\left(p_{n}^{2} \circ R, Y_{i}, z\right) d \mathcal{L}_{2} z d \phi_{n} R \\
& =\beta(n, 2)^{-1} \int_{G_{n}} \int_{E_{2}} N\left(p_{n}^{2} \circ R \circ l_{f}, W \cap Q, z\right) d \mathcal{L}_{2} z d \phi_{n} R \\
& \geqq \beta(n, 2)^{-1} \int_{G_{n}} \int_{E_{2}} S\left(p_{n}^{2} \circ R \circ f_{i},\left\{x \mid m_{f}(x) \in W\right\}, z\right) d \mathcal{L}_{2} z d \phi_{n} R .
\end{aligned}
$$

Proof of (7). For $z \in E_{2}$ we define

$$
g(z)=\sum_{A \in W,\left(p_{n}^{2} \circ R \circ l_{f}\right)(A)=z} L_{*}(f, A)
$$

and observe that

$$
\begin{gathered}
L_{*}(f, A)=\infty \text { for } A \in W-U, \\
g(z)=\infty \text { for } z \in\left(p_{n}^{2} \circ R \circ l_{f}\right)(W-U) .
\end{gathered}
$$

On the other hand if

$$
\begin{gathered}
z \in E_{2}-\left(p_{n}^{2} \circ R \circ l_{f}\right)(W-U), \\
S\left(p_{n}^{2} \circ R \circ f,\left\{x \mid m_{f}(x) \in W\right\}, z\right) \\
=S\left(p_{n}^{2} \circ R \circ f,\left\{x \mid m_{f}(x) \in U \cap W\right\}, z\right),
\end{gathered}
$$

and by (5) and Theorem 6.7 the second member of this equation is for $\mathcal{L}_{2}$ almost all such $z$ less than or equal to

$$
N\left(p_{n}^{2} \circ R \circ l_{f}, U \cap W \cap Q, z\right) \leqq g(z) .
$$

Proof of (8). From (7) we infer that

$$
\begin{aligned}
\beta(n, 2)^{-1} & \int_{G_{n}} \int_{E_{2}} S\left(p_{n}^{2} \circ R \circ f,\left\{x \mid m_{f}(x) \in W\right\}, z\right) d \mathcal{L}_{2} z d \phi_{n} R \\
& \leqq \beta(n, 2)^{-1} \int_{G_{n}} \int_{E_{2}}{ }_{A} \sum_{W,\left(p_{n}^{2} \circ R\right.} \sum_{\left.l_{f}\right)(A)=z} L_{* 2}(f, A) d \mathcal{L}_{2} z d \phi_{n} R
\end{aligned}
$$




$$
\begin{aligned}
& =\beta(n, 2)^{-1} \int_{G_{n}} \int_{E_{2}} \sum_{\left(p_{n}^{2} \circ R\right)(y)=z z} \sum_{A \in W, l_{f}(A)=y} L_{* 2}(f, A) d \mathcal{L}_{2} z d \phi_{n} R \\
& =\int_{E_{n}} \sum_{A \in W, l_{f}(A)=y} L_{* 2}(f, A) d \mathcal{F}_{n}^{2} y .
\end{aligned}
$$

REMARK. In case $n=3$ we can add the following three statements to the conclusions of Theorem 8.16:

(9) $L_{2}^{*}(f, A)=L_{* 2}(f, A)=\varepsilon_{2}(f, A)=0$ or 1 for $H_{f}^{2}$ almost all $A$ in $U$.

(10) $L_{2}^{*}(f, A)=L_{* 2}(f, A)=0$ or 1 or $\infty$ for $H_{f}^{2}$ almost all $A$ in $\mathscr{X}_{f}$.

(11) If $W$ is an open subset of $\mathcal{X l}_{f}$, then

$$
\sum_{A \in W, l_{f}(A)=y} L_{2}^{*}(f, A)=\sum_{A \in W, l_{f}(A)=y} L_{* 2}(f, A)
$$

is a non-negative integer or $\infty$ for $\mathcal{F C}_{3}^{2}$ almost all $y$ in $E_{3}$.

We observe that (9) implies (10) because

$$
L_{* 2}(f, A)=\infty \text { for } A \in \mathscr{X}_{f}-U,
$$

and that (10) implies (11) because $l_{f}$ maps sets of $H_{f}^{2}$ measure zero onto sets of $\mathfrak{H}_{3}^{2}$ measure zero.

In order to prove (9) it is sufficient to consider an open subset $V$ of $\mathscr{X}_{f}$ for which

$$
\lambda_{2}\left(f,\left\{x \mid m_{f}(x) \in V\right\}\right)<\infty
$$

and to show that

$$
L_{2}^{*}(f, A)=0 \text { for } H_{f}^{2} \text { almost all } A \text { in } V-Q .
$$

Suppose $\epsilon>0$. Since

$$
H_{f}^{2}(V \cap Q) \leqq \lambda_{2}\left(f,\left\{x \mid m_{f}(x) \in V\right\}\right)<\infty,
$$

we can select an open subset $W$ of $V$ such that

$$
V-Q \subset W, \quad H_{f}^{2}(W \cap Q)<\epsilon .
$$

Letting

$$
W^{\prime}=\left\{x \mid m_{f}(x) \in W\right\},
$$

we infer from (6) that

$$
\lambda_{2}\left(p_{3}^{2} \circ R \circ f, W^{\prime}\right)<\epsilon \text { for } R \in G_{8} .
$$

Choosing $R^{1}, R^{2}, R^{3} \in G_{3}$ so that 


$$
R^{1}(x)=x, \quad R^{2}(x)=\left(x_{2}, x_{3}, x_{1}\right), \quad R^{3}(x)=\left(x_{3}, x_{1}, x_{2}\right)
$$

whenever $x=\left(x_{1}, x_{2}, x_{3}\right) \in E_{3}$, we find that

$$
\lambda_{2}\left(f, W^{\prime}\right) \leqq \sum_{i=1}^{3} \lambda_{2}\left(p_{3}^{2} \circ R^{i} \circ f, W^{\prime}\right)<3 \epsilon .
$$

This conclusion can be justified by extending the fundamental inequality of Cesari [CE 2] from mappings of two-cells to mappings of arbitrary finitely triangulable subsets of $E_{2}$ into $E_{3}$. This extension presents no great difficulty.

It follows from Theorem 6.2 that

$$
\int_{V-Q} L_{2}^{*}(f, A) d H_{f}^{2} A \leqq \int_{W} L_{2}^{*}(f, A) d H_{f}^{2} A \leqq \lambda_{2}\left(f, W^{\prime}\right)<3 \epsilon .
$$

The arbitrary nature of $\epsilon$ implies that

$$
\begin{gathered}
\int_{V-Q} L_{2}^{*}(f, A) d H_{f}^{2} A=0, \\
L_{2}^{*}(f, A)=0 \text { for } H_{f}^{2} \text { almost all } A \text { in } V-Q .
\end{gathered}
$$

TheOREM 8.17. If $X$ is a 2-cell and $f \in C_{3}(X)$, then

$$
\begin{aligned}
L_{2}(f) & =\int_{\mathcal{X}_{f}} L_{2}^{*}(f, A) d H_{f}^{2} A=\int_{\mathcal{X}_{f}} L_{* 2}(f, A) d H_{f}^{2} A \\
& =\int_{E_{n}} \sum_{l_{f}(A)=y} L_{2}^{*}(f, A) d \mathcal{F}_{n}^{2} y=\int_{E_{n}} \sum_{l_{f}(A)=y} L_{* 2}(f, A) d \mathcal{F}_{n}^{2} y \\
& =\int_{E_{n}} \sum_{l_{f}(A)=y} L_{2}^{*}(f, A) d \mathcal{F}_{n}^{2} y=\int_{E_{n}} \sum_{l_{f}(A)=y} L_{* 2}(f, A) d \mathcal{F}_{n}^{2} y .
\end{aligned}
$$

Proof. By virtue of Theorem 6.2 each of the above seven numbers is less than or equal to $L_{2}(f)$ and greater than or equal to the last integral. Furthermore Theorem 8.16 implies that the last integral is greater than or equal to the 2-dimensional integralgeometric stable area of $f$, which equals $L_{2}(f)$.

\section{BIBLIOGRAPHY}

A. S. Besicovitch

B 1. On the fundamental geometrical properties of linearly measurable plane sets of points I, II, III, Math. Ann. vol. 98 (1927) p. 422; vol. 115 (1938) p. 296; vol. 116 (1939) p. 349.

B 2. On surfaces of minimum area, Proc. Cambridge Philos. Soc. vol. 44 (1948) p. 313. 
C. Caratheodory

C. Über das lineare Mass von Punktmengen-eine Verallgemeinerung des Längenbegriffs, Nachr. Ges. Wiss. Göttingen (1914) p. 404.

L. Cesari

CE 1. Su di un problema di analysis situs delle spazio ordinario, Rendiconti dell'Istituto Lombardo di Scienze e Lettere vol. 75 (1941) p. 267.

CE 2. Caratterizzazione analitica delle superficie continue di area finita secondo Lebesgue, Annali della Scuola Normale Superiore di Pisa (2) vol. 10 (1941) p. 253; vol. 11 (1942) p. 1.

CE 3. Sui punti di diramazione delle trasformazione continue e sull' area delle superficie in forma parametrica, Rendiconti di Math. e delle sue Applicazioni Roma (5) vol. 3 (1942) p. 37.

CE 4. Criteri di uguale continuita' ed applicazioni alla quadratura delle superficie, Annali della Scuola Normale Superiore di Pisa (2) vol. 12 (1943) p. 61.

CE 5. Una ugualianza fondamentale per l'area delle superficie, Atti della reale Academia d'Italia vol. 14 (1944) p. 891.

J. Douglas

DJ. Solution of the problem of Plateau, Trans. Amer. Math. Soc. vol. 33 (1931) p. 263.

C. H. DOWKER

D. Mapping theorems for noncompact spaces, Amer. J. Math. vol. 69 (1947) p. 200.

J. FAVARD

FA. Une défnition de la longueur et de l'aire, C. R. Acad. Sci. Paris vol. 194 (1932) p. 344.

H. FEDERER

F 1. Surface area. I, II, Trans. Amer. Math. Soc. vol. 55 (1944) p. 420.

F 2. The Gauss-Green Theorem, Trans. Amer. Math. Soc. vol. 58 (1945) p. 44.

F 3. Coincidence functions and their integrals, Trans. Amer. Math. Soc. vol. 59 (1946) p. 441.

F 4. The $(\phi, k)$ rectifiable subsets of $n$-space, Trans. Amer. Math. Soc. vol. 62 (1947) p. 114.

F 5. Dimension and measure, Trans. Amer. Math. Soc. vol. 62 (1947) p. 536.

F 6. Essential multiplicity and Lebesgue area, Proc. Nat. Acad. Sci. U.S.A. vol. 34 (1948) p. 411.

F 7. Hausdorff measure and Lebesgue area, Proc. Nat. Acad. Sci. U.S.A. vol. 37 (1951) p. 90.

F. HAUSDORFF

H. Dimension und aiisseres Mass, Math. Ann. vol. 79 (1918) p. 157.

W. HuRewicz and H. Wallman

HW. Dimension theory, Princeton Mathematical Series, Princeton University Press, 1941.

H. LEBESGUE

L. Intégrale, longueur, aire, Annali di Matematica Pura ed Applicata vol. 7 (1902) p. 231.

E. J. McShane

MCS. Parametrization of saddle surfaces with application to the problem of Plateau, Trans. Amer. Math. Soc. vol. 35 (1933) p. 716.

E. F. MOORE

MEF 1. Convexly generated k-dimensional measures, Proceedings of the American Mathematical Society vol. 2 (1951) p. 597. 
MEF 2. Density ratios and $(\phi, 1)$ rectifiability in n-space, Trans. Amer. Math. Soc. vol. 69 (1950) p. 324.

C. B. MORREY, JR.

MCB 1. A class of representations of manifolds. I, II, Amer. J. Math. vol. 55 (1933) p. 683; vol. 56 (1934) p. 275.

MCB 2. An analytic characterization of surfaces of finite Lebesgue area. I, II, Amer. J. Math. vol. 57 (1935) p. 692; vol. 58 (1936) p. 313.

A. P. MORSE

M. A theory of covering and differentiation, Trans. Amer. Math. Soc. vol. 55 (1944) p. 205.

A. P. Morse AND H. Federer

MF. $\quad$ Some properties of measurable functions, Bull. Amer. Math. Soc. vol. 49 (1943) p. 270.

A. P. MoRse AND J. F. Randolph

MR. The $\phi$ rectifiable subsets of the plane, Trans. Amer. Math. Soc. vol. 55 (1944) p. 236.

H. RADEMACHER

RA. $\quad$ Über partielle und totale Differenzierbarkeit. I, Math. Ann. vol. 79 (1919) p. 340 .

T. RADO

R 1. The problem of least area and the problem of Plateau, Math. Zeit. vol. 32 (1930) p. 763.

R 2. On the derivative of the Lebesgue area of continuous surfaces, Fund. Math. vol. 30 (1938) p. 34.

R 3. On absolutely continuous transformations in the plane, Duke Math. J. vol. 4 (1938) p. 189.

R 4. On continuous mappings of Peano spaces, Trans. Amer. Math. Soc. vol. 58 (1945) p. 420.

R 5. On surface area, Proc. Nat. Acad. Sci. U.S.A. vol. 31 (1945) p. 102.

R 6. Length and area, Amer. Math. Soc. Colloquium Publications, vol. 30, New York, 1948.

A. SARD

S. The equivalence of n-measure and Lebesgue measure in $E_{n}$, Bull. Amer. Math. Soc. vol. 49 (1943) p. 758.

H. WHITNEY

W. On totally differentiable and smooth functions, Pacific Journal of Mathematics vol. 1 (1951) p. 143.

BROWN UNIVERSITY 